\title{
PHOTONIC MICROCAVITIES AND PHOTONIC SPONGES BASED ON SILICON COLLOIDS
}

Ph.D. THESIS BY

Michal TyMCZENKo

Departamento de Física Aplicada

Universidad Politécnica de VAlencia 

Ph.d. Thesis on:

Photonic Microcavities AND PHOTONIC SPONGES BASED ON SILICON COLLOIDS

Author:

Michae Tymczenko

Directors:

Prof. Francisco J. Meseguer Rico

Doc. Roberto Fenollosa

Center:

Centro de Tecnologías Físicas: Acústica,

MATERIAles y Astrofísica

Unidad Asociada: Insituto de Ciencia de Materiales

DE MADRID-CSIC/UPV

Department:

Física APLiCAda

Universidad Politécnica de VAlencia

Valencia, April 2010 



\section{Acknowledgements}

I would like to express my gratitude to all those who gave me the possibility to complete this thesis. I want to thank Professor Francisco Meseguer who supervised and offered me the opportunity to do my Ph.D. in Valencia at Universidad Politécnica de Valencia. Roberto - who put me in contact with Prof. Meseguer in Japan, his patience with my questions, exchange of ideas and for introducing me to the world of philosophical physics where an elephant can be simplified to a silicon sphere. Fernando for introducing me to buñolero and La Tomatina, for helping me with LabView and other non-described projects. Jose Manuel who helped me as a translator during my first four months in Valencia. Isabel for forcing me to speak castellano. Ana for helping me with finding items in the lab and making the CVD depositions. Hector for helping me with MatLab and ${ }_{A} \mathrm{~T}_{\mathrm{E}} \mathrm{X}$, when strange error codes appeared. The technicians from Servicio de Microscopia - Manolo, Jose and Merche, who endured my requests for tilting and rotating samples, not to mention the time they spent for preparing ion milling the TEM samples. Saïd from University of Valencia for sessions of HRTEM. Paco Cervera for teaching me to how use a lathe. Vicente Fornes for letting me use the facilities from ITQ, Jose Luis Jordá for help with the X-ray diffraction measurement. David Busquets for letting me use facilities from Materials Department and thanks to all those people, who are not mentioned here but have helped me in one or another way. Ana Cross and Andres Cantarero for help with Raman measurements. Elivra Bonet for help with the paperwork. My special thanks to my wife Patrizia who has proofread my texts and has been a great support in all aspects throughout the work towards my Ph.D.

I gratefully acknowledge Tekn. dr Marcus Wallenbergs Stiftelse för utbildning i internationellt industriellt företagande. This thesis would not have been possible without their financial support. 


\begin{abstract}
Silicon $(\mathrm{Si}$ ) is a material of paramount importance in both microelectronics and photonics. The semiconducting properties of silicon are behind the fundamental concepts of most of the electronic devices as diodes and transistors. The concept of integration has allowed processing very small devices, reaching the nano metric size. The high refractive index of silicon allows confining light in structures whose size is in the micrometric range. This is the case of photonic devices such as waveguides and cavities. Normally both electronic and photonic devices are based on planar technology, i.e.: they have a flat topology, being this a source of losses. It is well known that spherical cavities confine light much stronger than planar cavities. This $\mathrm{PhD}$ thesis reports on the development of a new type of spherical microparticles we call as Silicon Colloids. Because of their spherical shape, high refractive index and smooth surface, they work as optical microcavities with well defined resonating modes in the near infrared range. This thesis will report on the synthesis, structural and optical properties of silicon colloids with diameter from 0.5 to $3.5 \mu \mathrm{m}$. Silicon colloids may facilitate development of high quality factor optical microcavities with strong light confinement effects, allowing integration of electronic and photonic devices such as a p-n junction into a single colloidal particle. This thesis will report also on silicon colloids as building blocks of Photonic Sponges formed by disordered networks of silicon microspheres of different sizes that scatter light strongly in a wide range of wavelengths.
\end{abstract}




\section{Resumen}

El silicio es un material de suma importancia en microelectrónica y en fotónica. Las propiedades semiconductoras del silicio están detrás de los conceptos que gobiernan el funcionamiento de la mayoría de los dispositivos electrónicos como los diodos y los transistores. El concepto de integración ha permitido procesar dispositivos muy pequeños, llegando a alcanzar un tamaño nanométrico. El alto índice de refracción del silicio permite confinar la luz en estructuras de tamańo micrométrico. Este es el caso de dispositivos fotónicos tales como las guías de onda y las cavidades. Usualmente, tanto los dispositivos fotónicos como los electrónicos están basados en la tecnología planar, es decir poseen una topología plana, siendo ésto una fuente de pérdidas. Es bien conocido que las cavidades esféricas confinan la luz con más eficiencia que las cavidades planares. Esta tesis trata sobre el desarrollo de un nuevo tipo de micropartículas esféricas que llamamos Coloides de Silicio. Debido a su forma esférica, su alto índice de refracción y su suave superficie, estas partículas funcionan como microcavidades ópticas con modos resonantes bien definidos en el infrarrojo cercano. La tesis reporta sobre la síntesis, y las propiedades estructurales y ópticas de los coloides de silicio con diámetro entre 0.5 y 3.5 micrómetros. Los coloides de silicio pueden facilitar el desarrollo de microcavidades de alto factor de calidad con alta eficiencia de confinamiento de la luz, y permitir la integración de dispositivos electrónicos y fotónicos tales como una unión p-n en una sola partícula coloidal. Esta tesis reporta también sobre los coloides de silicio como elementos integrantes de las Esponjas Fotónicas, las cuales están formadas por una red desordenada de microesferas de silicio de diferentes tamaños, e interaccionan con la luz fuertemente en un ancho rango de longitudes de onda. 


\section{Resum}

El silici és un material de vital importància en microelectrònica i en fotònica. Les propietats semiconductores del silici estan darrere dels conceptes que governen el funcionament de la majoria dels dispositius electrònics com els diodes i els transistors. El concepte d'integració ha permès processar dispositius molt xicotets, que poden arribar a tindre un tamany nanomètric. L'alt índex de refracció del silici permet confinar la llum en estructures de tamany micromètric. Aquest es el cas de dispositius fotònics com les guies dona i les cavitats. Usualment, tant els dispositius fotònics com els electrònics estan basats en la tecnologia planar, és a dir, posseeixen una topologia plana, siguent aquesta una font de pèrdues. És ben conegut que les cavitats esfèriques confinen la llum amb més eficiència que les cavitats planars. Aquesta tesi tracta sobre el desenvolupament d'un nou tipus de micropartícules esfèriques que anomenem Coloids de Silici. Degut a la seva forma esfèrica, al seu alt índex de refracció i a la seva suau superfície, aquestes partícules funcionen com a micro-cavitats òptiques amb modes ressonants ben definits en l'infraroig proper. La tesi reporta sobre la síntesis, i les propietats estructurals i òptiques dels coloids de silici amb diàmetre comprés entre $0.5 \mathrm{i}$ 3.5 micròmetres. Els coloids de silici podrien facilitar el desenvolupament de microcavitats d'alt factor de qualitat amb una alta eficiència de confinament de la llum, i permetrien la integració de dispositius electrònics i fotònics com per exemple una unió p-n en una sola partícula. Aquesta tesi reporta també sobre els coloids de silici com a elements integrants de les Esponges Fotòniques, les quals estan formades per una xarxa desordenada de microesferes de silici de diferents tamanys, i interaccionen amb la llum fortament en un ampli rang de longituds d'ona. 


\section{Contents}

1 Introduction $\quad 13$

1.1 Optical microcavities . . . . . . . . . . . . . . . 13

1.1.1 Parameters of a microcavity . . . . . . . . . 14

1.1.2 Types of microcavities . . . . . . . . . . . . . 15

1.1.2.1 Fabry-Pérot type cavities . . . . . . 15

1.1.2.2 Photonic crystal cavities . . . . . . . 16

1.1.2.3 Whispering gallery modes optical cavities 18

1.2 Our approach . . . . . . . . . . . . . . . . 19

References ...................... 24

2 Synthesis of silicon colloids $\quad 25$

2.1 Introduction . . . . . . . . . . . . . . . . 25

2.2 CVD setup and fabrication procedure . . . . . . . . 26

2.2.1 How do silicon colloids form? . . . . . . . . . 29

2.3 Conclusions . . . . . . . . . . . . . . . . . 30

References .............................. 31

3 Structural properties of silicon colloids 33

3.1 Introduction . . . . . . . . . . . . . . . . 33

3.2 Sphere diameter distribution of silicon colloids . . . . . . 34

3.3 Crystallinity and hydrogen content of silicon colloids . . 40

3.3.1 X-Ray powder Diffraction (XRD) . . . . . . . 41

3.3.2 Raman spectroscopy . . . . . . . . . . . . . . 44

3.3.3 High Resolution Transmission Electron Microscopy (HRTEM) . . . . . . . . . . 46

3.3.4 Hydrogen content of silicon colloids . . . . . . . 47 
3.3.4.1 Fourier Transform InfraRed Spectroscopy 47

3.3.4.2 Mass Spectrometry ......... . 50

3.4 Surface morphology . . . . . . . . . . . . . . . . . . . 52

3.5 Conclusions . . . . . . . . . . . . . . . . 53

References ...................... 55

4 Optical properties of silicon colloids $\quad 57$

4.1 Introduction . . . . . . . . . . . . . . 57

4.2 Theory about light scattering by particles . . . . . . . 58

4.2.1 Introduction . . . . . . . . . . . . . . . . 58

4.2.2 The light trapping phenomenon . . . . . . . . 59

4.2 .3 The Mie theory . . . . . . . . . . . . . . . . 61

4.2.4 The influence of the refractive index on the resonance phenomena . . . . . . . . . 68

4.3 Experimental results . . . . . . . . . . . . . . . 70

4.3.1 Optical properties of single silicon colloids . . . . 70

4.3.1.1 Experimental setup . . . . . . . . . 70

4.3.1.2 The obtained spectra and their fit to Mie theory ............... 71

4.3.2 Optical properties of photonic sponges . . . . . 81

4.3.2.1 The influence of the sphere size distribution 83

4.3.2.2 The influence of the filling fraction . . . 86

4.4 Conclusions . . . . . . . . . . . . . . . . 93

References ...................... 94

5 General conclusions $\quad 95$

5.1 Conclusions . . . . . . . . . . . . . . . . . . 95

5.2 Towards future . . . . . . . . . . . . . . . . 95

$6 \quad$ List of publications $\quad 97$

6.1 Scientific Articles in International Journals . . . . . . . . 97

6.2 Cover Images in International Journals . . . . . . . . . . 98

6.3 International Conferences and Symposia . . . . . . . . . . 99

6.4 Domestic Conferences and Symposia . . . . . . . . . . 99

6.5 Patents . . . . . . . . . . . . . . 100

$\begin{array}{ll}\text { List of figures } & 103\end{array}$

$\begin{array}{ll}\text { Appendix } & 103\end{array}$ 
$\begin{array}{ll}\text { A Sphere in the box model } & 105\end{array}$

B Colloidal Crystal Wires 107

B.1 Introduction . . . . . . . . . . . . . . . 107

B.2 Experimental setup . . . . . . . . . . . . . . 111

B.2.1 Fabrication of macroporous silicon membrane . . 111

B.2.2 Fabrication of colloidal wires . . . . . . . . . . 112

B.3 Results and discussion . . . . . . . . . . . . . . 115

B.4 Conclusions . . . . . . . . . . . . . . . . . . . 119

References ..................... 120

C Acronyms 125 


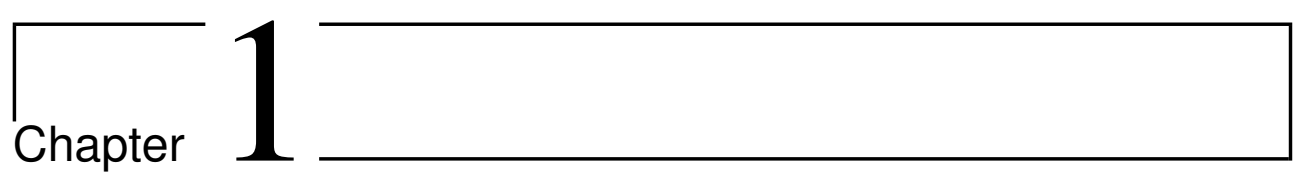

\section{Introduction}

The object of research of this thesis is in the framework of optical microcavities. This field has experienced recently an increasing development $[1,2]$ thanks to the improvement of methods that allow fabricating sophisticated micro and nano-structures [3, 4]. Optical microcavities constitutes currently a hot topic that has raised interesting competitions regarding different aspects of science and technology between researchers from all over the world.

In this chapter, a short introduction to the field of optical microcavities will be given. This introduction includes the basic mechanisms of light confinement, the most important parameters defining a microcavity as well as the different types of microcavities. Finally we will explain our approach to the field that is based on silicon colloids.

\section{$1.1 \quad$ Optical microcavities}

The most important role of an optical cavity, also called optical resonator, is the confinement of light. This can be achieved by virtue of two different schemes:

- by reflection of light at a single interface, for instance a metallic surface or by total internal reflection at a boundary between two dielectrics.

- by destructive interference of waves in periodically patterned microstructures as it occurs in a multilayer Bragg reflector or in a photonic crystal. 
The size of an optical cavity is usually larger than the wavelength of the confined light. However, an optical microcavity is characterized by having a size that is of the same order of magnitude as the wavelength of light, i.e. its size is in the micrometer or sub micrometer range.

\subsubsection{Parameters of a microcavity}

The ideal cavity would confine light indefinitely (without losses) and would resonate at a precise single frequency; i.e.: with a delta like resonance. Deviation from this value is described by the quality factor or $Q$ factor. $Q$ factor is dimensionless and it describes how well the cavity confines the energy. It is defined in equation (1.1):

$$
Q=2 \pi \frac{\text { Total stored energy }}{\text { Energy loss per cycle }}
$$

Another way of writing the $Q$ factor is by means of the ratio between the cavity resonant frequency $f$ and the full width at half-maximum (FWHM) of the cavity mode $\Delta f$ :

$$
Q=\frac{f}{\Delta f}
$$

Therefore, the higher the $Q$, the more energy is confined in the cavity, and the more pronounced is the peak associated to the resonance in the frequency spectrum. Other parameter frequently used to describe a microcavity is the effective volume $\left(V_{e f f}\right) . V_{e f f}$ is defined as the spatial integral of the field intensity in the mode, normalized to unity at the field maximum (see equation (1.3)):

$$
V_{e f f}=\frac{\int \varepsilon(\mathbf{r})|\mathbf{E}(\mathbf{r})|^{2} d^{3} \mathbf{r}}{\max \left[\varepsilon(\mathbf{r})|\mathbf{E}(\mathbf{r})|^{\mathbf{2}}\right]}
$$

where $\varepsilon(\mathbf{r})$ is the dielectric constant and $\mathbf{E}(\mathbf{r})$ is the electric field.

The effective mode volume depends on the particular resonant mode and it gives an idea of the volume the electromagnetic energy occupies in the microcavity.

Finally, other important parameter of a microcavity is the Free Spectral Range (FSR). FSR is the distance in the frequency or wavelength space without any mode. For an empty standing-wave resonator of length $L$ as illustrated in figure 1.1, it can be calculated by using equation (1.4). 


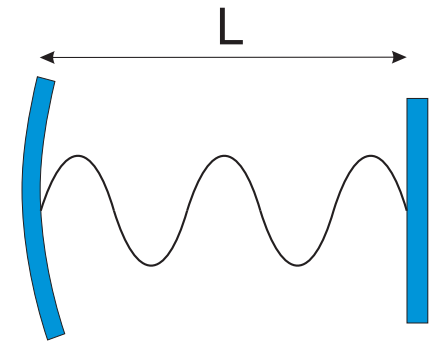

(a)

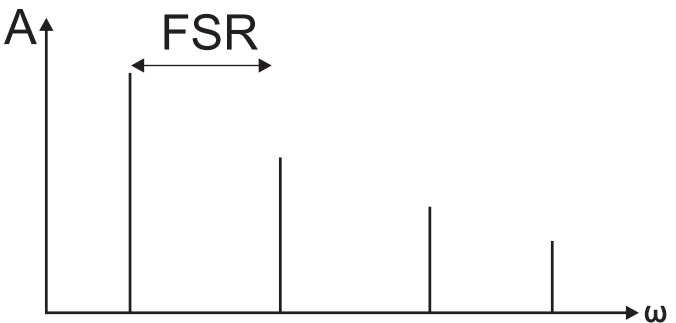

(b)

Figure 1.1: (a) A laser cavity consisting of a plane mirror and a concave mirror. The cavity will have several longitudinal modes which are integral values of wavelength. (b) Cavity modes (vertical lines) in the frequency space. The distance between consecutive modes is the Free Spectral Range (FSR).

$$
F S R=\frac{c}{2 L}
$$

where $c$ is the speed of light in vacuum.

The FSR of a resonator is important for instance whenever a luminescent material, having a wide spectral emission, is introduced inside it, because the emitted energy will be divided between all the modes whose frequency is included in the emission range. If the FSR is large enough so that only one mode coincides with the emission spectrum, laser emission may be greatly enhanced.

\subsubsection{Types of microcavities}

The microcavities can be divided into three basic families [2]: FabryPérot, whispering gallery mode and photonic crystal. They will be described below.

\subsubsection{Fabry-Pérot type cavities}

A Fabry-Pérot cavity (after Charles Fabry and Alfred Pérot) is composed of two parallel mirrors (plane or concave), which are brought into close proximity so that only a few wavelengths of light can fit in between them. Perhaps one of the most common application of a Fabry-Pérot cavity is a semiconductor laser, like that shown in figure 1.2. 


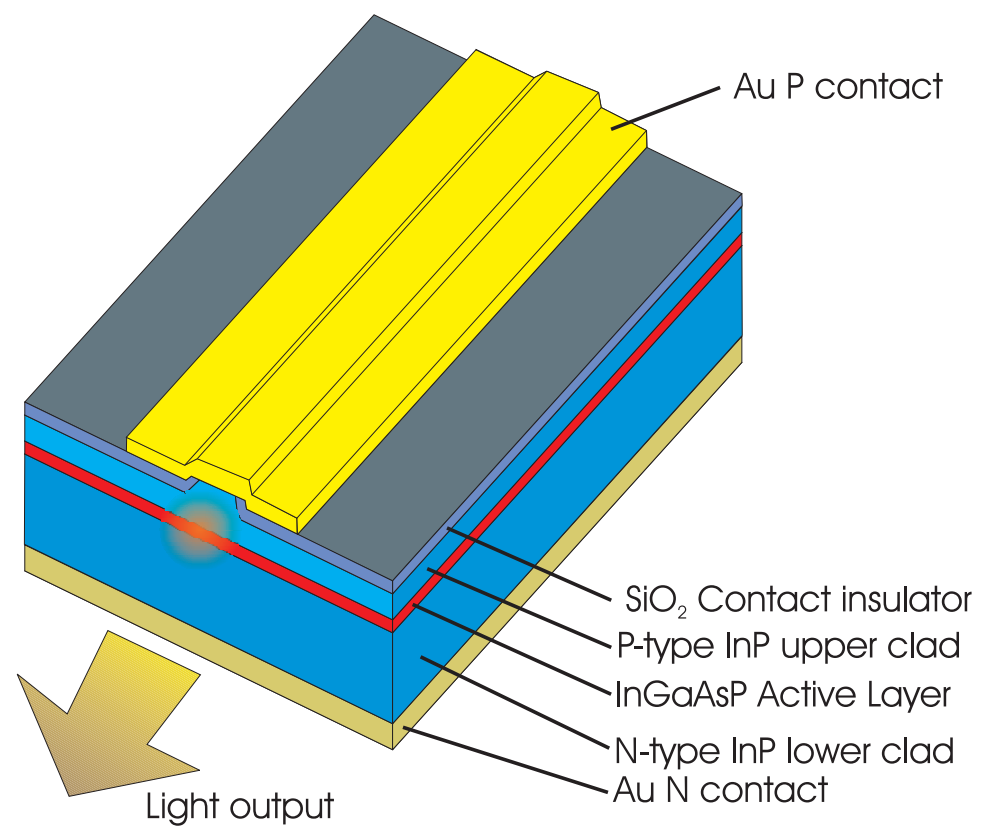

Figure 1.2: Fabry-Pérot edge emitting semiconductor laser. The cleaved edges act as mirrors by high index contrast.

The semiconductor laser was simultaneously invented at three different labs: IBM [5], General Electric [6, 7] and MIT Lincoln Laboratory [8]. Further, the semiconductor laser was improved by introducing the Distributed Feedback laser (DFB), which consists of replacing the cleaved facet mirrors by a corrugated layered grating. Other type of the FabryPérot semiconductor laser is the Vertical Cavity Surface Emitting Laser (VCSEL).

\subsubsection{Photonic crystal cavities}

The periodic arrangement of ions in a lattice gives rise to the energy band structure in semiconductors. Energy bands control the motion of charged carriers through the crystal. Similarly, in a photonic crystal (PC), the periodic arrangement of the refractive index controls how photons are able to move through the crystal. Photonic crystals are dielectric materials in which the refractive index is periodic in one, two or three dimensions of space $(1 \mathrm{D}, 2 \mathrm{D}$ or $3 \mathrm{D})$ as depicted in figure 1.3. 


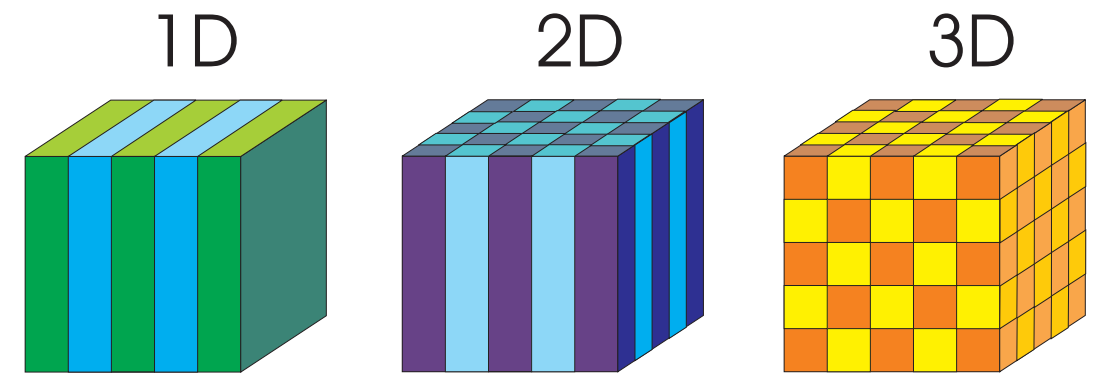

Figure 1.3: An illustrative example of (from left to right) 1D, 2D and 3D photonic crystal. Different colors represent materials with different dielectric properties.

By periodically varying the refractive index, photons react to the refractive index contrast in an analogous manner as electrons do when confronted with a periodic potential of ions. Each one results in a range of allowed energies and a band structure characterized by an energy gap or photonic band gap. Depending on its wavelength, the light is allowed to propagate in what is called modes. Groups of allowed propagation modes form bands. Those wavelengths in which photons can not propagate are called photonic band gaps.

A photonic band gap may allow to suppress spontaneous emission and to achieve localization of light, as first proposed by E. Yablonovitch [9] and S. John [10]. A point defect may act as a microcavity (see figure 1.4) with confinement of the electromagnetic field in all directions. Microcavities based on photonic crystals $[11,12]$ can provide extremely small modal volumes [13] and very high $Q$ values [14-18]. To date, the highest $Q$ value measured for a photonic crystal cavity is $2.5 \times 10^{6}[19]\left(V_{\text {eff }}=1.4(\lambda / n)^{3}\right)$ where $\lambda$ is the resonant wavelength and $n$ is the refractive index of the slab. Photonic crystals can be made of silicon [20], silica [21, 22] or gallium arsenide (GaAs) [23]. One dimensional (1D) photonic crystals are very similar to Fabry-Pérot structures and they have applications in thin film optics where they are used in high reflection mirrors. 2D PC have had so far less applications. One commercial application of 2D PC concerns improving efficiency of light emitting diodes (LED) [24]. 3D PC are still far from commercialization. 


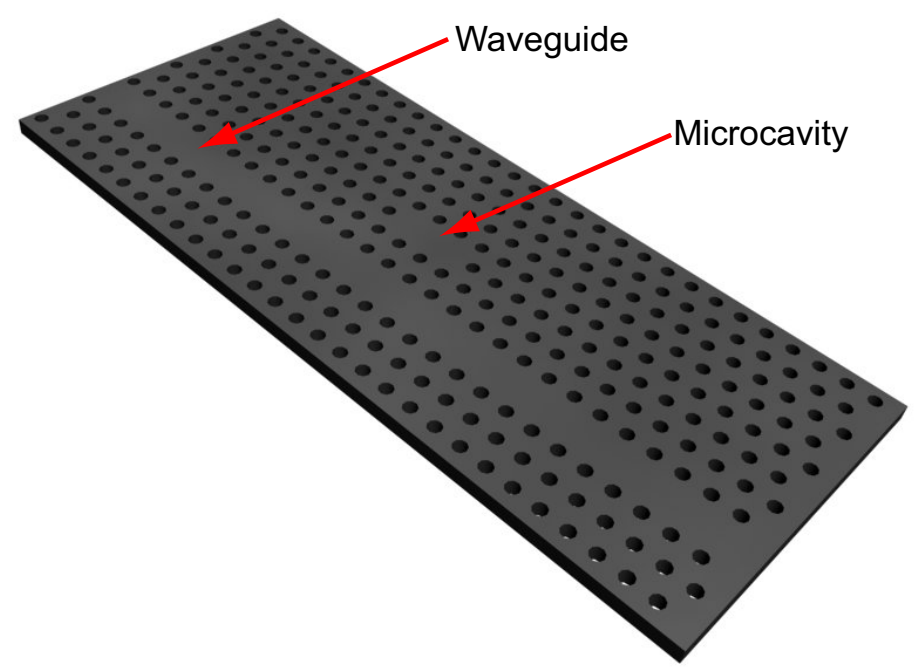

Figure 1.4: Schematic of a $2 \mathrm{D}$ photonic crystal slab made of silicon. Light is confined vertically by the high refractive index contrast between silicon and air, and horizontally by the band gap created by the periodically arranged holes. Holes are created by etching the silicon slab. By etching the holes in the silicon slab in a particular way a microcavity and a waveguide can be created as indicated by the arrows [25].

\subsubsection{Whispering gallery modes optical cavities}

Whispering gallery resonators are optical resonators where the electromagnetic radiation is confined by total internal reflection effect in high refractive index materials with circular geometry. Roughly speaking, it is as if light performed a round trip through the perimeter of the resonator with many total internal reflections at the boundary between the resonator and the surrounding medium. This resembles the whispering gallery acoustics modes first noticed by Lord Rayleigh in Saint Pauls cathedral in London. Whispering gallery cavities may be divided into 2D and 3D. 2D structures have shapes like that of a disk [26, 27], a toroid [28] (figure 1.5(a)) and a gear [29]. 3D microcavities are usually highly spherical (figure 1.5(b)). 


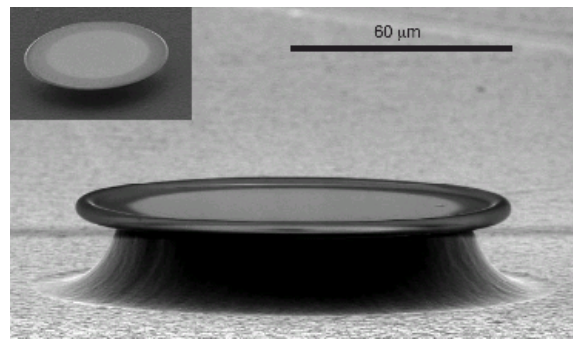

(a)

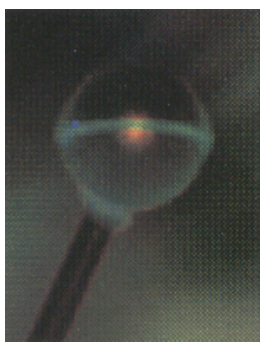

(b)

Figure 1.5: Different types of whispering gallery mode cavities (a) Ultra-high-Q toroid microcavity [28]. (b) Erbium doped silica sphere approximately $70 \mu m$ in diameter with visible green luminescence from Erbium upconversion. In the image the fiber optic stem that supports the sphere is visible [30].

The most common whispering gallery resonators are made of silica or quartz. Silica microspheres [31] were the first to be studied [32-36]. Surface quality is of great importance for maximizing the $Q$ value in spherical whispering gallery mode cavities. The highest reported $Q$ value to date is $7.2 \times 10^{9}$ at a wavelength of $850 \mathrm{~nm}$ in a silica microsphere [37] $\left(V_{\text {eff }}=3000 \mu \mathrm{m}^{3}\right.$, diameter $\left.=680 \mu \mathrm{m}\right)$, where the $Q$ value measured is close to the theoretical one. High $Q$ silica microspheres are usually fabricated by heating the tip of a telecommunication optical fibre with a $\mathrm{CO}_{2}$ laser. As silica is highly absorptive at $10.6 \mu \mathrm{m}$ thus it can be readily melted. Surface tension will force the melted tip to form a near spherical shape. Drops of liquid with an almost perfect sphere shape have been also investigated [38-40].

\subsection{Our approach}

The main contribution of this thesis consists of a new proposal of photonic microcavity, namely a whispering gallery mode microcavity consisting of silicon colloids. They are highly spherical silicon particles with a diameter from 0.5 to $3.5 \mu \mathrm{m}$ approximately, and they have a very smooth surface. This makes them work as optical microcavities. Moreover, the fact that silicon has a very high refractive index $(n=3.5)$ allows a very efficient confinement in spite of their small size, which is of the same order of magnitude as the wavelength of light in the near infrared range. Silicon colloids posses notable advantages comparing to other spherical 
resonators based on different dielectrics like silica or poly-styrene. Perhaps one of the most interesting properties is the fact that they allow building up electronic circuitry, so that and optoelectronic device can be developed in a single silicon colloid, for instance a p-n junction. The goals of this thesis are, therefore, the study of different aspects of silicon colloids. Such goals include:

- The synthesis of silicon colloids by Chemical Vapor Deposition (chapter 2).

- The study of their structural properties: size, crystallinity, surface roughness, etc (chapter 3).

- Optical characterization (chapter 4). This is perhaps the most important goal and it includes measurements of colloids one by one for proving that they work, in fact, as photonic microcavities. In addition, we study the optical properties of photonic sponges consisting of random networks of silicon colloids.

During the period of the thesis, a research that is not directly related to the topic of Silicon Colloids was also performed. Such a research activity is about colloidal crystal wires and it yielded notable results that were published in Advanced Materials [41]. Appendix B includes all the details of that research. 


\section{Bibliography}

[1] L. Pavesi and D. J. Lockwood (Eds.). Silicon Photonics. Springer, Berlin, Germany, 2004.

[2] Kerry J. Vahala. "Optical microcavities". Nature, 424(6950):839846, 2003.

[3] Shigeki Takahashi, Katsuyoshi Suzuki, Makoto Okano, Masahiro Imada, Takeshi Nakamori, Yuji Ota, Kenji Ishizaki, and Susumu Noda. "Direct creation of three-dimensional photonic crystals by a top-down approach". Nature Materials, 8(9):721-725, 2009.

[4] Kanna Aoki, Hideki T. Miyazaki, Hideki Hirayama, Kyoji Inoshita, Toshihiko Baba, Kazuaki Sakoda, Norio Shinya, and Yoshinobu Aoyagi. "Microassembly of semiconductor three-dimensional photonic crystals". Nature Materials, 2(2):117-121, 2003.

[5] Marshall I. Nathan, William P. Dumke, Gerald Burns, Jr. Frederick H. Dill, and Gordon Lasher. "Stimulated emission of radiation from GaAs p-n junctions". Applied Physics Letters, 1(3):62-64, 1962.

[6] Jr. Nick Holonyak and S. F. Bevacqua. "Coherent (visible) light emission from $\mathrm{Ga}\left(\mathrm{As}_{1-x} \mathrm{P}_{x}\right)$ junctions". Applied Physics Letters, 1(4):82-83, 1962.

[7] R. N. Hall, G. E. Fenner, J. D. Kingsley, T. J. Soltys, and R. O. Carlson. "Coherent Light Emission From GaAs Junctions". Physical Review Letters, 9(9):366, 1962.

[8] T. M. Quist, R. H. Rediker, R. J. Keyes, W. E. Krag, B. Lax, A. L. McWhorter, and H. J. Zeigler. "Semiconductor maser of GaAs". Applied Physics Letters, 1(4):91-92, 1962.

[9] Eli Yablonovitch. "Inhibited Spontaneous Emission in Solid-State Physics and Electronics". Physical Review Letters, 58(20):2059, 1987.

[10] Sajeev John. "Strong localization of photons in certain disordered dielectric superlattices". Physical Review Letters, 58(23):2486, 1987.

[11] C. Reese. "High-Q photonic crystal microcavities fabricated in a thin GaAs membrane". J. Vac. Sci. Technol. B, 19:2749-2752, 2001. 
Introduction

[12] T. D. Happ. "Enhanced light emission of $\operatorname{In}_{x} \mathrm{Ga}_{1-x}$ As quantum dots in a two- dimensional photonic-crystal defect microcavity". Phys. Rev. B, 66:041303-041303, 2002.

[13] O. Painter, R. K. Lee, A. Scherer, A. Yariv, J. D. O’Brien, P. D. Dapkus, and I. Kim. "Two-Dimensional Photonic Band-Gap Defect Mode Laser". Science, 284(5421):1819-1821, 1999.

[14] J. Vuckovic, M. Loncar, H. Mabuchi, and A. Scherer. "Design of photonic crystal microcavities for cavity QED". Phys. Rev. E, 6501:016608-016608, 2002.

[15] K. Srinivasan, P. Barclay, O. Painter, J. Chen, C. Cho, and C. Gmachl. "Experimental demonstration of a high quality factor photonic crystal microcavity". Appl. Phys. Lett., 2002.

[16] K. Srinivasan and O. Painter. "Momentum space design of high-Q photonic crystal optical cavities". Opt. Exp., 10:670-684, 2002.

[17] Takasumi Tanabe, Masaya Notomi, Eiichi Kuramochi, Akihiko Shinya, and Hideaki Taniyama. "Trapping and delaying photons for one nanosecond in an ultrasmall high-Q photonic-crystal nanocavity". Nature Photonics, 1(1):49-52, 2007.

[18] Bong-Shik Song, Susumu Noda, Takashi Asano, and Yoshihiro Akahane. "Ultra-high-Q photonic double-heterostructure nanocavity". Nature Materials, 4(3):207-210, 2005.

[19] Yasushi Takahashi, Hiroyuki Hagino, Yoshinori Tanaka, Bong-Shik Song, Takashi Asano, and Susumu Noda. "High-Q nanocavity with a 2-ns photon lifetime". Opt. Express, 15(25):17206-17213, 2007.

[20] Alvaro Blanco, Emmanuel Chomski, Serguei Grabtchak, Marta Ibisate, Sajeev John, Stephen W. Leonard, Cefe Lopez, Francisco Meseguer, Hernan Miguez, Jessica P. Mondia, Geoffrey A. Ozin, Ovidiu Toader, and Henry M. van Driel. "Large-scale synthesis of a silicon photonic crystal with a complete three-dimensional bandgap near 1.5 micrometres". Nature, 405(6785):437-440, 2000.

[21] H. Miguez, C. Lopez, F. Meseguer, A. Blanco, L. Vazquez, R. Mayoral, M. Ocana, V. Fornes, and A. Mifsud. "Photonic crystal properties of packed submicrometric $\mathrm{SiO}_{2}$ spheres". Applied Physics Letters, 71(9):1148-1150, 1997. 
[22] H. Miguez, F. Meseguer, C. Lopez, A. Mifsud, J. S. Moya, and L. Vazquez. "Evidence of FCC Crystallization of $\mathrm{SiO}_{2}$ Nanospheres". Langmuir, 13(23):6009-6011, 1997.

[23] Kenji Ishizaki and Susumu Noda. "Manipulation of photons at the surface of three-dimensional photonic crystals". Nature, 460(7253):367-370, 2009.

[24] Jonathan J. Wierer, Michael R. Krames, John E. Epler, Nathan F. Gardner, Joel R. Wendt, Mihail M. Sigalas, Steven R. J. Brueck, Dong Li, and Michael Shagam. "III-nitride LEDs with photonic crystal structures". In Light-Emitting Diodes: Research, Manufacturing, and Applications IX, volume 5739, pages 102-107, San Jose, CA, USA, 2005. SPIE.

[25] Takashi Asano, Masamitsu Mochizuki, Susumu Noda, Makoto Okano, and Masahiro Imada. "A Channel Drop Filter Using a Single Defect in a 2-D Photonic Crystal Slab-Defect Engineering With Respect to Polarization Mode and Ratio of Emissions From Upper and Lower Sides". J. Lightwave Technology, 21(5):1370, 2003.

[26] N. C. Frateschi and A. F. J. Levi. "Resonant modes and laser spectrum of microdisk lasers". Applied Physics Letters, 66(22):29322934, 1995.

[27] T. Baba, P. Fujita, A. Sakai, M. Kihara, and R. Watanabe. "Lasing characteristics of GaInAsP-InP strained quantum-well microdisk injection lasers with diameter of 2-10 $\mu \mathrm{m}$ ". Photonics Technology Letters, IEEE, 9(7):878-880, 1997.

[28] D. K. Armani, T. J. Kippenberg, S. M. Spillane, and K. J. Vahala. "Ultra-high-Q toroid microcavity on a chip". Nature, 421:925-928, 2003.

[29] Masayuki Fujita and Toshihiko Baba. "Microgear laser". Applied Physics Letters, 80(12):2051-2053, 2002.

[30] M. Cai, O. Painter, K. J. Vahala, and P. C. Sercel. "Fiber-coupled microsphere laser". Optics Letters, 25(19):1430-1432, 2000.

[31] V. B. Braginsky, M. L. Gorodetsky, and V. S. Ilchenko. "Qualityfactor and nonlinear properties of optical whispering-gallery modes". Phys. Lett. A, 137:393-397, 1989. 
[32] V. LefevreSeguin and S. Haroche. "Towards cavity-QED experiments with silica microspheres". Mat. Sci. Eng. B, 48:53-58, 1997.

[33] M. L. Gorodetsky, A. A. Savchenkov, and V. S. Ilchenko. "Ultimate Q of optical microsphere resonators". Opt. Lett., 21:453-455, 1996.

[34] D. W. Vernooy, V. S. Ilchenko, H. Mabuchi, E. W. Streed, and H. J. Kimble. "High-Q measurements of fused-silica microspheres in the near infrared". Opt. Lett., 23(4):247-249, 1998.

[35] J. C. Knight. "Mapping whispering-gallery modes in microspheres with a near-field probe". Opt. Lett., 20:1515-1517, 1995.

[36] D. W. Vernooy, A. Furusawa, N. P. Georgiades, V. S. Ilchenko, and H. J. Kimble. "Cavity QED with high-Q whispering gallery modes". Phys. Rev. A, 57:R2293-R2296, 1998.

[37] D. W. Vernooy, V. S. Ilchenko, H. Mabuchi, E. W. Streed, and H. J. Kimble. "High-Q measurements of fused-silica microspheres in the near infrared". Opt. Lett., 23(4):247-249, 1998.

[38] H.-B. Lin, J. D. Eversole, and A. J. Campillo. "Continuouswave stimulated Raman scattering in microdroplets". Opt. Lett., 17(11):828-830, 1992.

[39] "Microcavity enhanced Raman gain". Optics Communications, 133(1-6):287 - 292, 1997.

[40] Rudolf Penndorf. "Mie scattering coefficient for water droplets in air". Journal of the Atmospheric Sciences, 13(2):219-220, 1956.

[41] Michael Tymczenko, Lluis F. Marsal, Trifon Trifonov, Isabelle Rodriguez, Fernando Ramiro-Manzano, Josep Pallares, Angel Rodriguez, Ramon Alcubilla, and Francisco Meseguer. "Colloidal Crystal Wires". Advanced Materials, 20(12):2315-2318, 2008. 
${ }_{\text {Chapter }} 2$

\section{Synthesis of silicon colloids}

\subsection{Introduction}

Chemical Vapor Deposition (CVD) is an important technique used to produce high-purity, high-performance solid materials. CVD can be used to deposit materials like silicon nitride, silicon dioxide, and metals - copper, aluminium, nickel, tungsten, titanium, molybdenum and tantalum. The CVD technique is used wildly in the semiconductor industry to grow thin films.

In a CVD process, the substrate or the item to be coated is exposed to one or more precursor gases, which react and/or decompose on the substrate surface to produce the desired deposit. For depositing silicon, the precursor gases can be silane, disilane or dichlorosilane. For depositing metals, precursor gases such as Copper Formate, Triethyl Aluminum or tungsten hexafluoride $\left(W F_{6}\right)$ can be used. In a typical CVD process a precursor gas is decomposed by means of a heat source. In the simplest version of CVD, the heat source is a tubular oven. This is called hot-wall thermal CVD. In more advanced systems the decomposition energy is provided by plasma - Plasma Enhanced Chemical Vapor Deposition (PECVD) or by ultraviolet light (UV). PECVD allows deposition at lower temperatures, which is often critical in the manufacture of semiconductors. In PECVD, the plasma is generally created by Radio Frequency (RF), Alternating Current (AC) or direct current (DC) discharge between two electrodes.

We have used hot wall thermal CVD with disilane as the precursor gas for silicon colloids fabrication. In this chapter the fabrication parameters and the procedure will be described. 


\subsection{CVD setup and fabrication procedure}

The CVD setup used to synthesize silicon colloids is illustrated in figure 2.1. It consists of a glass artwork, a vacuum pump, a liquid nitrogen trap, a reactor and a disilane cylinder. The glass artwork connects all the previously mentioned parts. Air is removed from the system by means of a vacuum pump. The trap is used to increase the final vacuum and to remove unreacted gases from the setup. The reactor is the place where disilane decomposition and silicon colloids nucleation and growing occurs.

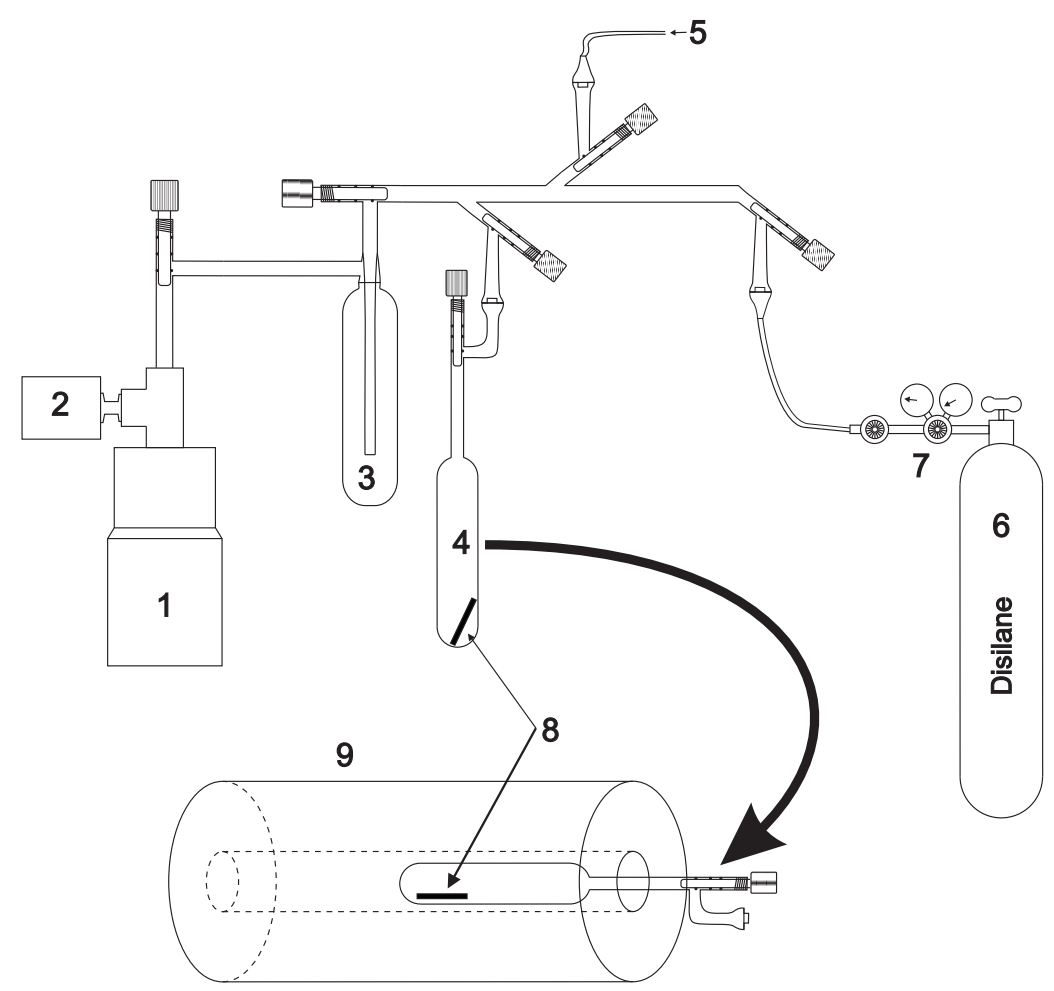

Figure 2.1: CVD setup used to fabricate silicon colloids. The main components of the setup are: 1) Membrane pump with Turbo Molecular Pump 2) Vacuum gauge 3) Liquid Nitrogen Trap 4) Reactor 5) Nitrogen gas inlet 6) Disilane cylinder 7) Pressure valve 8) Substrate. 9) Tubular oven.

The fabrication procedure of silicon colloids consists of three main steps:

1. introducing the disilane into the reactor, 
2. dissociating the disilane gas by introducing the reactor into a tubular oven, and

3. extracting the remaining gases and the substrate containing the silicon colloids from the reactor.

The procedure starts with inserting a clean substrate into the reactor - a quartz, glass or silicon sheet can be used for this purpose. The substrate facilitates later on the removal of the silicon colloids as they will deposit on to it. The reactor with the substrate inside is attached to the glass artwork and all the CVD setup is put under vacuum by means of the turbo molecular pump. Once a good vacuum condition is reached, typically a pressure of $5 \cdot 10^{-6}$ mbar, the reactor is cooled down with liquid nitrogen and a certain amount of disilane is released into the system. We have chosen to keep constant this amount to a value of about $30 \mathrm{mg}$ in all of our experiments. Taking into account the volume of the reactor is $45 \mathrm{ml}$, the pressure of the gas at room temperature is around $26 \mathrm{kPa}$.

All the released disilane gas will condensate inside the reactor because disilane becomes liquid at temperatures below $-14.5{ }^{\circ} \mathrm{C}$. After that, the reactor is closed, removed from liquid nitrogen, detached from the glass artwork and inserted into a preheated tubular oven as depicted in figure 2.2(a) and 2.2(b). The heat from the oven induces dissociation of the disilane and nucleation and growing of silicon colloids inside the gas. During this process the colloids will deposit everywhere onto the walls of the reactor but mainly on the substrate by gravity (some colloids will attach on the underside of the substrate as well). In parallel, an epitaxial layer of silicon with thickness below $1 \mu \mathrm{m}$ will grow both onto the substrate and on the inside walls of the reactor.

Depending on the time the reactor remains in the oven and the used decomposition temperature, disilane will dissociate completely or partially and different amounts of silicon colloids with distinct average diameters will be synthesized. We have used decomposition times up to 60 minutes and temperatures from $375{ }^{\circ} \mathrm{C}$ to $800{ }^{\circ} \mathrm{C}$.

The disilane decomposition process stops with removing the reactor from the oven and letting it reach room temperature. At this stage residual gases like disilane and hydrogen remain inside the reactor together with the already grown silicon colloids. Such residual gases should be eliminated carefully before opening the reactor to air because disilane ignites spontaneously in air. For this purpose, the reactor is reattached to 


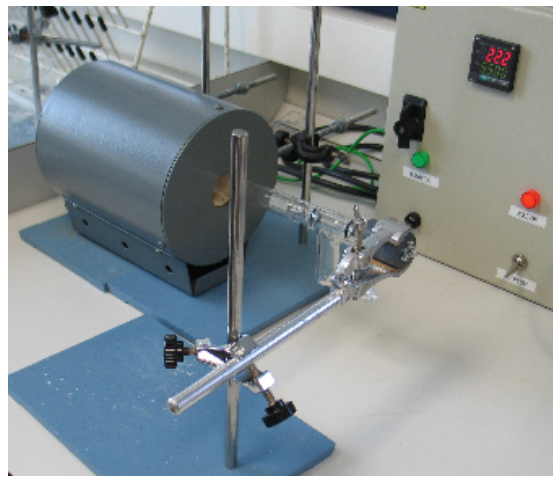

(a)

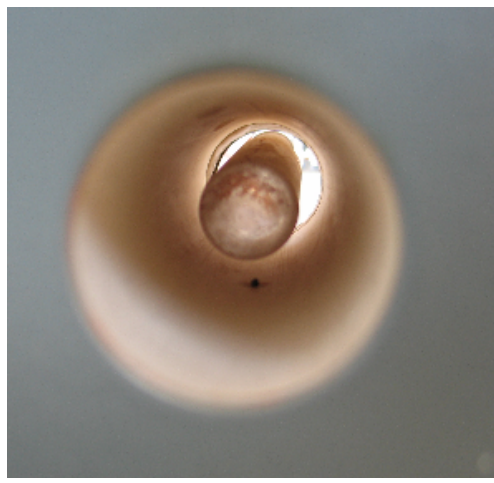

(b)

Figure 2.2: Tubular oven used to fabricate silicon colloids. (a) Reactor inserted into the oven. (b) Inside view of the tubular oven. The reactor with the substrate is allocated centrally. The dark spot in the center of the image is the thermocouple providing temperature measurements.

the glass artwork, and all the CVD setup, except the reactor that is still closed, is put under vacuum again. Then, the trap (nr. 3 in figure 2.1) is cooled down with liquid nitrogen and the reactor is opened. Making so forces the remaining disilane gas to condensate inside the trap. Finally, disilane is deactivated by flushing with nitrogen gas while the setup is being opened to ambient atmosphere. After this, the reactor can be safely removed from the glass artwork and the substrate with the obtained silicon colloids can be extracted from the reactor.

Scanning Electron Microscopy images (SEM, Jeol JSM 6400/JSM 5410) of the obtained silicon colloids are showed in figure 2.3. They can be found isolated (figure 2.3(a)) or in clusters of several or many units (figure 2.3(b)) depending on the disilane decomposition temperature and time. We observed that temperatures below $400{ }^{\circ} \mathrm{C}$ produced isolated colloids while higher temperatures gave rise to aggregations of many colloids. We call such aggregations Photonic Sponges (PS) due to their particular optical scattering properties as we will see in chapter 4 . The images of figure 2.3 show that the obtained colloids are polydisperse with sizes around 2 micrometers. This will be analyzed more in detail in chapter 3. The colloids are highly spherical, that is why we also call them silicon microspheres, and they have a very smooth surface although some of them show nicks and defects presumably due to the fact that they were in contact with other colloids during the synthesis process. The structural properties, i.e. spherical condition, surface roughness, etc. are very 
important as far as optical properties are concerned and they will be described more in deep in chapter 3.

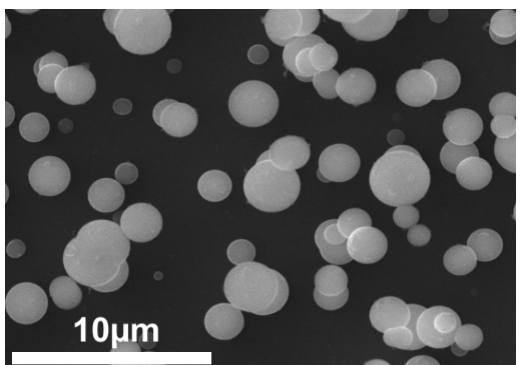

(a)

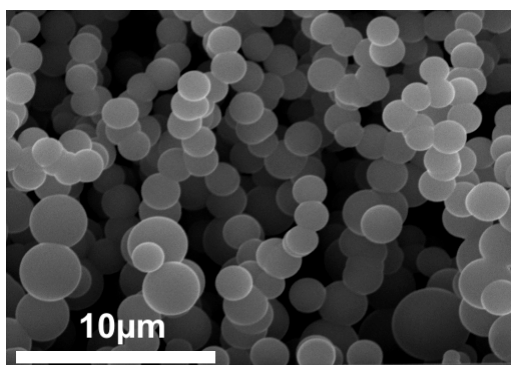

(b)

Figure 2.3: Scanning electron microscopy images of silicon colloids obtained by the hot wall thermal CVD process. A variety of silicon colloids can be obtained by changing the disilane decomposition time and temperature. Silicon colloids are polydisperse in size and can be found in clusters or separately. (a) Colloids obtained at $385{ }^{\circ} \mathrm{C}$ during 10 minutes. (b) Colloids obtained at $500{ }^{\circ} \mathrm{C}$ during 1 hour.

\subsubsection{How do silicon colloids form?}

The chemistry involved in the disilane or silane decomposition process to obtain silicon is very complicated and much research has been devoted to this topic [1-5]. So far, such research activities have been focused on obtaining high quality thin layers of silicon, and the formation of particles in the gas phase has been something to avoid. In spite of the big efforts realized, it seems many questions regarding the decomposition mechanisms still remain unknown. In this thesis, we have not focused on the formation mechanisms of silicon colloids, but rather on their optical properties. The formation mechanisms constitute a very complex problem involving chemistry in gas and solid phases, and nucleation and diffusion of particles at the same time, etc. Particularly, the thermodynamic parameters we use are quite different from that ones utilized by researchers that try to grow epitaxial silicon. We believe, this constitutes the topic of another thesis itself and it is currently an object of research. Nevertheless, some hints regarding the chemistry of disilane are given below. In particular, it is worth mentioning that some parallels can be made to the growing process of silica colloids in liquid [6-8].

$$
\mathrm{Si}_{2} \mathrm{H}_{6} \rightarrow 3 \mathrm{H}_{2}+2 \mathrm{Si}
$$


This chemical reaction occurs in the gas phase and onto any substrate in contact with the gas at the same time. In the first case, it produces $S i_{x} H_{y}$ clusters that finally become silicon colloids after hydrogen desorption. In the second case it gives rise to an epitaxial silicon layer. These two processes are illustrated schematically in figure 2.4 .

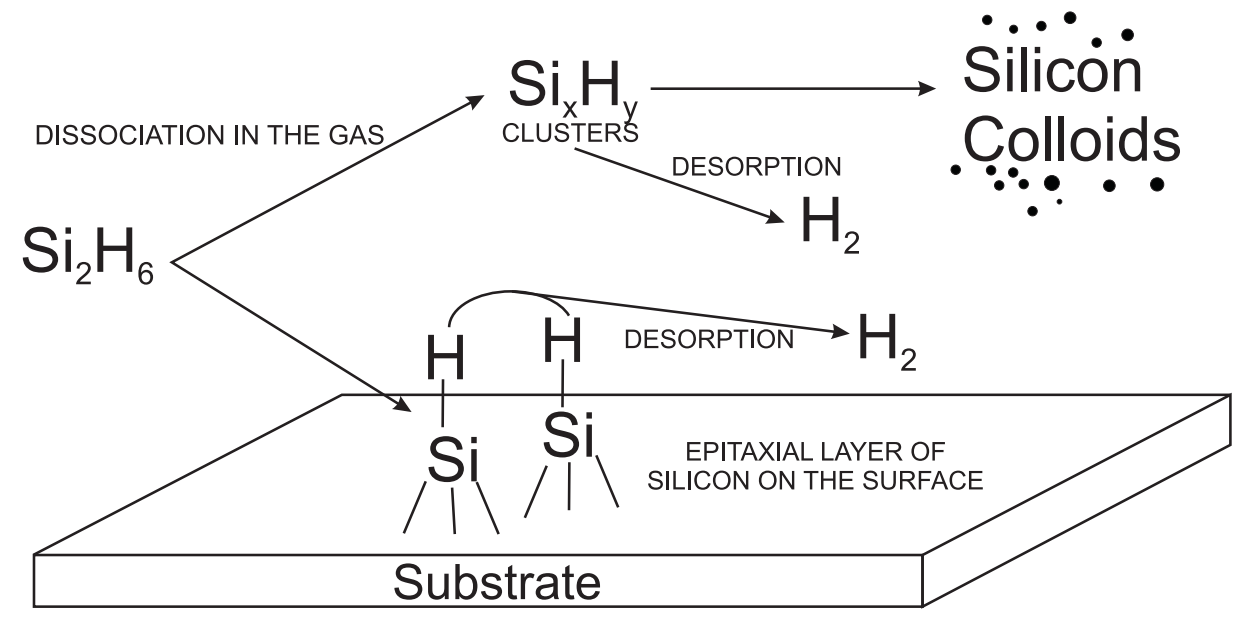

Figure 2.4: Simplified schematics of disilane dissociation. In parallel to the creation of silicon colloids an epitaxial layer of silicon will cover the substrate.

\subsection{Conclusions}

In this chapter the CVD fabrication procedure for obtaining silicon colloids was described. By changing the CVD parameters like time and temperature, silicon colloids of different configurations can be obtained: single silicon spheres, clusters or an aggregation of many colloids. We have introduced the term Photonic Sponge as a name for the aggregations of many silicon colloids. The mechanism of formation of silicon colloids still remains unknown, and it is currently an object of research. 


\section{Bibliography}

[1] Joseph M. Jasinski and Stephen M. Gates. "Silicon chemical vapor deposition one step at a time: fundamental studies of silicon hydride chemistry". Accounts of Chemical Research, 24(1):9-15, 1991.

[2] Y. Suda, D. Lubben, T. Motooka, and J. E. Greene. "Adsorption and thermal dissociation of disilane $\left(\mathrm{Si}_{2} \mathrm{H}_{6}\right)$ on $\mathrm{Si}(100) 2 \times 1$ ". Journal of Vacuum Science Technology A: Vacuum, Surfaces, and Films, 8(1):61-67, 1990.

[3] D. Lubben, R. Tsu, T. R. Bramblett, and J. E. Greene. "Mechanisms and kinetics of $\mathrm{Si}$ atomic-layer epitaxy on $\mathrm{Si}(001) 2$ x 1 from $\mathrm{Si}_{2} \mathrm{H}_{6}$ ". Journal of Vacuum Science Technology A: Vacuum, Surfaces, and Films, 9(6):3003-3011, 1991.

[4] Y. Suda, D. Lubben, T. Motooka, and J. E. Greene. "Thermal and photostimulated reactions on $\mathrm{Si}_{2} \mathrm{H}_{6}$-adsorbed $\mathrm{Si}(100) 2$ x 1 surfaces: Mechanisms of Si film growth by atomic-layer epitaxy". Journal of Vacuum Science Technology B: Vacuum, Surfaces, and Films, $7(5): 1171-1175,1989$.

[5] John J. Boland. "Role of hydrogen desorption in the chemical-vapor deposition of Si(100) epitaxial films using disilane". Phys. Rev. B, 44(3):1383-1386, Jul 1991.

[6] Werner Stöber, Arthur Fink, and Ernst Bohn. "Controlled growth of monodisperse silica spheres in the micron size range". Journal of Colloid and Interface Science, 26(1):62-69, 1968.

[7] C. Jeffrey Brinker and George W. Scherer. Sol-Gel Science: The Physics and Chemistry of Sol-Gel Processing. Academic Press, 1990.

[8] Horacio E. Bergna. The Colloid Chemistry of Silica. American Chemical Society Publication, 1994. 
Chapter 3

\section{Structural properties of silicon colloids}

\subsection{Introduction}

Structural properties of silicon colloids such as size, crystallinity, surface roughness, etc. are very important because they have a direct impact on their optical properties. For instance, a smooth surface is the key factor for the colloids to be able to act as optical microcavities. A silicon colloid with a rough surface would destroy the resonances, specially those having a high quality factor. Other structural properties like the sphere size influence strongly the wavelength values at which optical resonances (Mie modes) occur. The type of silicon, amorphous or polycrystalline, also influences the optical resonances: while the polycrystalline phase does not absorb light in the near infrared, the amorphous phase may absorb some light at this wavelength region, what would reduce the quality factor of the resonant modes.

In this chapter the structural properties of silicon colloids are described and analyzed. These properties are sphere diameter distribution, crystallization, hydrogen content and surface morphology. They have been studied by different techniques which are described briefly in each section: Scanning Electron Microscopy (SEM), Raman spectroscopy, High Resolution Transmission Electron Microscopy (HRTEM), X-Ray powder Diffraction (XRD), Fourier Transform Infra Red spectroscopy (FTIR), Atomic Force Microscopy (AFM) and Mass Spectrometry. 


\subsection{Sphere diameter distribution of silicon colloids}

Silicon colloids are polydisperse in size as we observed in the SEM images of section 2.2 (see figure 2.3). Generally speaking, the colloids obtained by using the synthesis parameters ranges detailed in the previous chapter, i.e. disilane decomposition times up to 60 minutes and disilane decomposition temperatures from $400{ }^{\circ} \mathrm{C}$ to $800{ }^{\circ} \mathrm{C}$, posses a diameter of approximately 1.2 micrometers and a size dispersion width of approximately 1 micrometer. We have studied the sphere diameter distribution for the following disilane decomposition temperatures: $400{ }^{\circ} \mathrm{C}, 500{ }^{\circ} \mathrm{C}, 550{ }^{\circ} \mathrm{C}$, $600{ }^{\circ} \mathrm{C}, 700{ }^{\circ} \mathrm{C}$ and $800{ }^{\circ} \mathrm{C}$. The decomposition time was in all these cases 60 minutes because at this time the particles growing process has terminated completely, and all the particles have been deposited onto the the substrate and onto the walls of the reactor. This fact was verified by checking the light scattered by a laser beam as it travels through the reactor where the disilane decomposition is occurring (shown in figure 3.5). One can see such laser path while silicon colloids are still floating in the gas due to the scattering of light by the particles, but once no colloids remain in the gas, the laser path can not be seen anymore. The sphere diameter distributions are shown in figure 3.1.

These distributions were obtained by the following procedure: first, the substrate containing a layer of deposited silicon colloids is cleaved. As the thickness of this layer is not uniform, we always cleave the substrate through the line where the silicon colloids layer is the thickest. This way, the cross section of the layer can be observed easily. Secondly, several high magnification SEM images, typically from 30 to 50, at different positions of the silicon colloids layer cross section are recorded. Finally a MATLab algorithm (source code downloaded from MATlab central and adopted for multiple images) using circular Hough transform [1, 2] is used to find the diameter of the spheres in the SEM images as showed in figure 3.2. Taking into account the error associated to the scanning electronic microscope and how the MATLab algorithm works, the error of the obtained diameter values is estimated to $\pm 40 \mathrm{~nm}$. Average sphere size diameter measurement were repeated three times for different samples with the same deposition temperature and time, and a good reproducibility of the results was attained. 


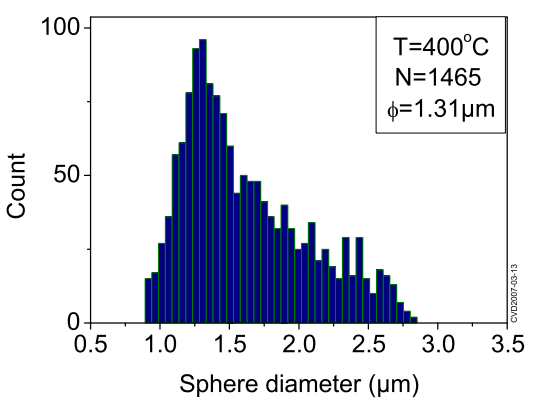

(a)

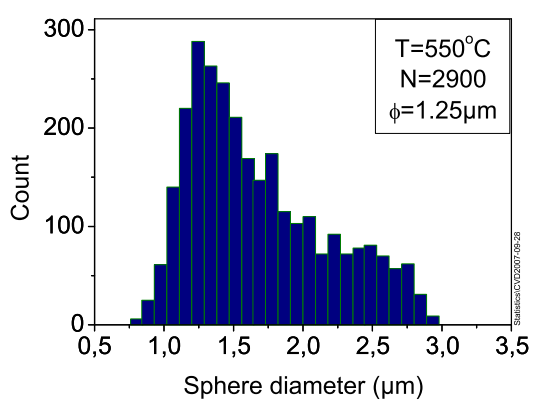

(c)

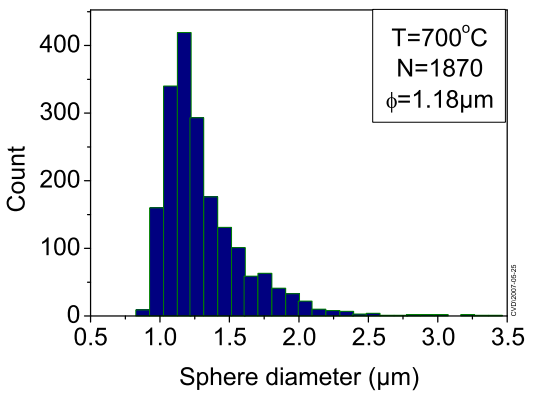

(e)

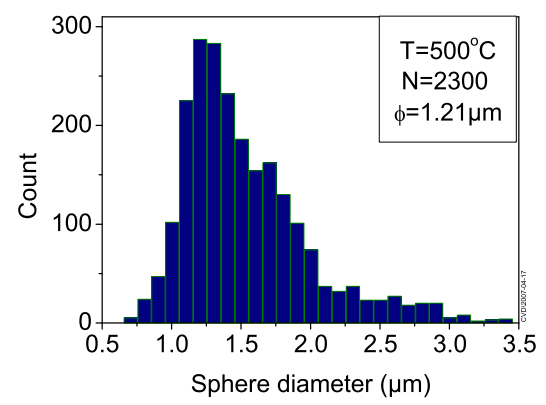

(b)

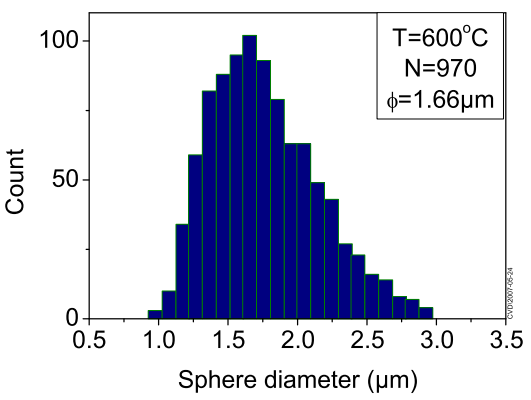

(d)

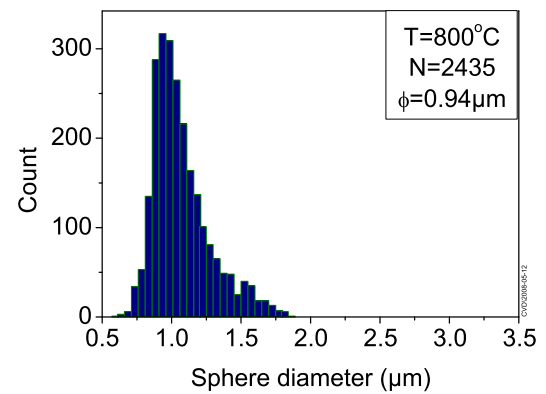

(f)

Figure 3.1: Histograms illustrating the sphere size distribution for silicon colloids synthesized by using a decomposition time of 60 minutes and a temperature of (a) $400{ }^{\circ} \mathrm{C}$, (b) $500{ }^{\circ} \mathrm{C}$, (c) $550{ }^{\circ} \mathrm{C}$, (d) $600{ }^{\circ} \mathrm{C}$, (e) $700{ }^{\circ} \mathrm{C}$ and (f) $800{ }^{\circ} \mathrm{C}$. The insets indicate the decomposition temperature $\mathrm{T}$, the number of spheres $\mathrm{N}$, used for obtaining the histogram and the mode, $\phi$, for the data set. 


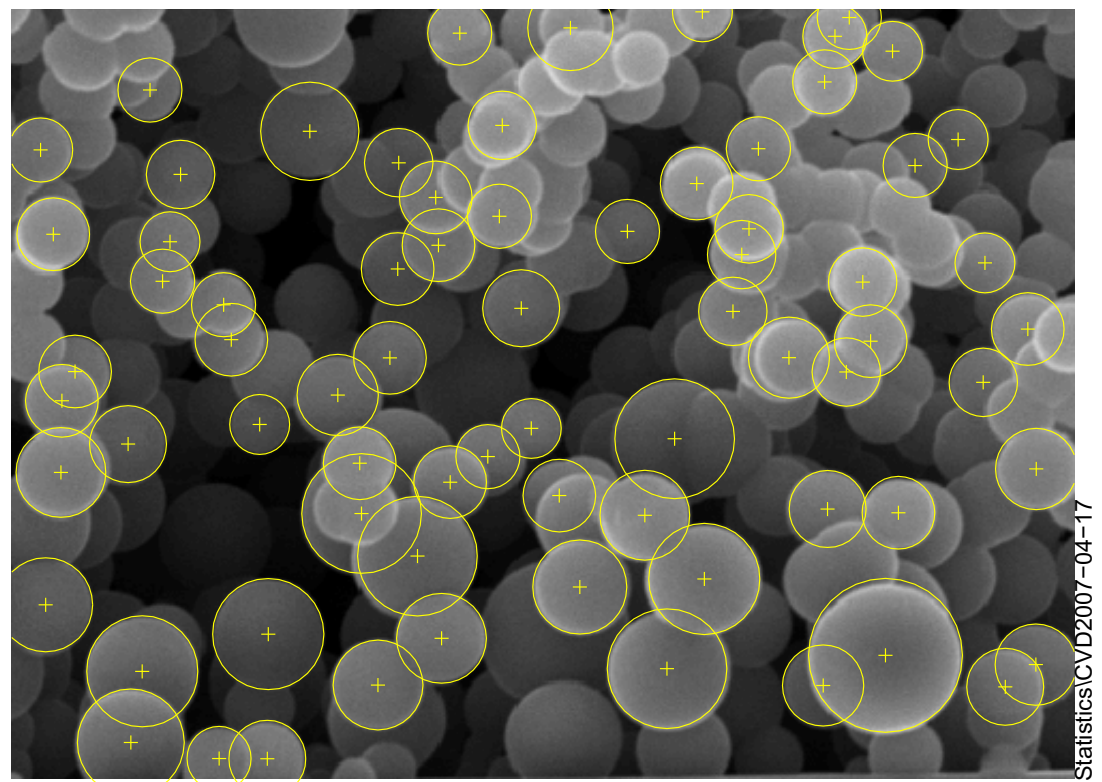

Figure 3.2: A SEM image of silicon colloids processed with a MATLab script using Hough transform to find the position and diameter of the single spheres. Found spheres are encircled in yellow.

Several points regarding the sphere diameter distributions of figure 3.1 should be emphasized:

1. No matter the decomposition temperature, all the distributions are biased to the right, i.e. to higher sphere diameter values.

2. There is a decomposition temperature between $550{ }^{\circ} \mathrm{C}$ and $700{ }^{\circ} \mathrm{C}$ where the sphere diameter mode achieves the highest value. This fact can be better seen by plotting the mode value of the distributions against temperature (figure 3.3).

3. The distribution width decreases substantially as the decomposition temperature approaches $800{ }^{\circ} \mathrm{C}$.

We have not found a definite explanation of these facts yet. This would require a precise knowledge of the silicon colloids synthesis process. Nevertheless, we would like to point out some aspects that may contribute to the explanation of the observed facts:

1. After disilane reaches the decomposition temperature, nucleation and growing of colloids occur at the same time. This results in 


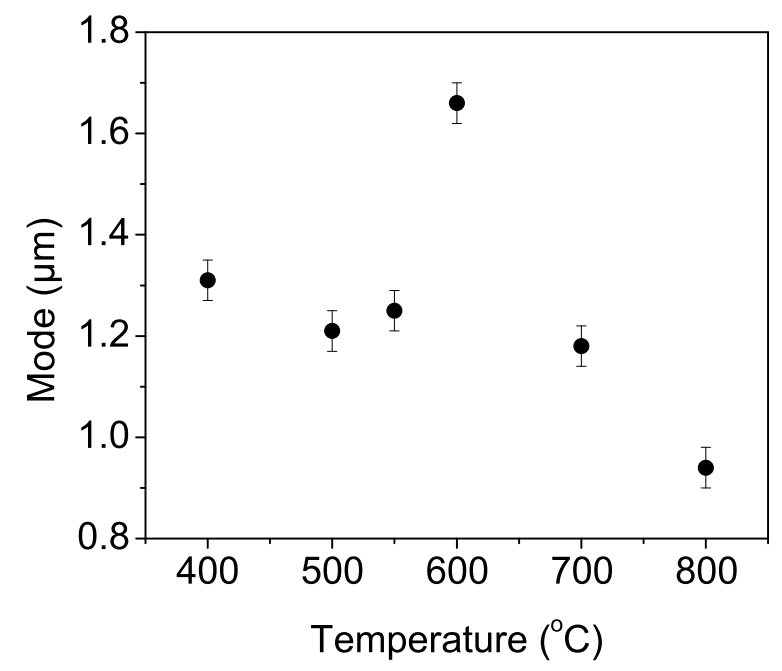

Figure 3.3: Mode as a function of deposition temperature. The largest mode is obtained around $600{ }^{\circ} \mathrm{C}$.

colloids that nucleated at different times and contributes to broaden the size distribution. In order to get mono-disperse colloids it would be desirable to separate the nucleation from the growing process.

2. There is a high density of particles at the early stages of the decomposition process. This favours that individual particles adhere to each other and give rise to bigger clusters that finally become silicon colloids. This could be an explanation of the observed biased distributions towards bigger sizes.

3. Disilane concentration varies as the decomposition process evolves. At the beginning we have $100 \%$ disilane and it decreases as decomposition takes place. However we have verified that the asymmetric dispersion of sizes is already present at the early decomposition stages. In figure 3.4(a) a comparison the sphere size distribution is showed for decomposition at a temperature of $500{ }^{\circ} \mathrm{C}$ for 4 minutes and 60 minutes. Therefore, this should not be the main reason of size poly-dispersion. 


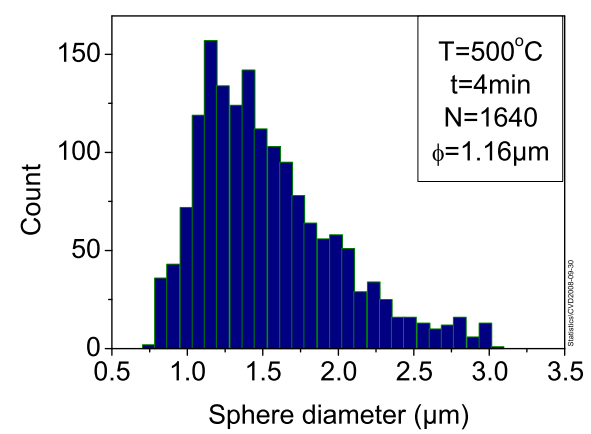

(a)

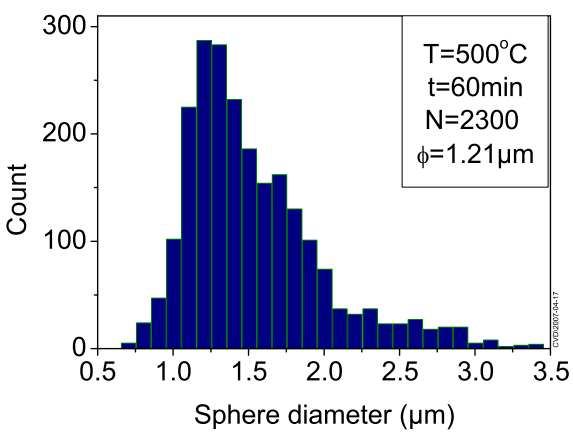

(b)

Figure 3.4: Histogram comparison between deposition at $500^{\circ} \mathrm{C}$ (a) 4 minutes and (b) 60 minutes. The insets indicate the decomposition temperature $T$, deposition time in minutes $t$, the number of spheres $N$, used for obtaining the histogram and the mode, $\phi$ for the data set.

4. The temperature of the reactor is not uniform. This is due to the temperature gradient of the tubular oven (from the hottest point in the center the temperature drops rapidly, as much as $30{ }^{\circ} \mathrm{C}$ per centimeter towards the muzzle) and to the fact that a part of the reactor is out of the oven, i.e. at room temperature. Therefore, a convection current is created by the temperature difference between the hot and the cold part of the reactor. Such convection current will cause that silicon colloids move in a pattern illustrated with arrows in figure 3.5. We actually observed such convection current by monitoring the light path of the red laser as shown in figure 3.5. In the figure a photograph of a part of the reactor where the laser path is visible is presented. 


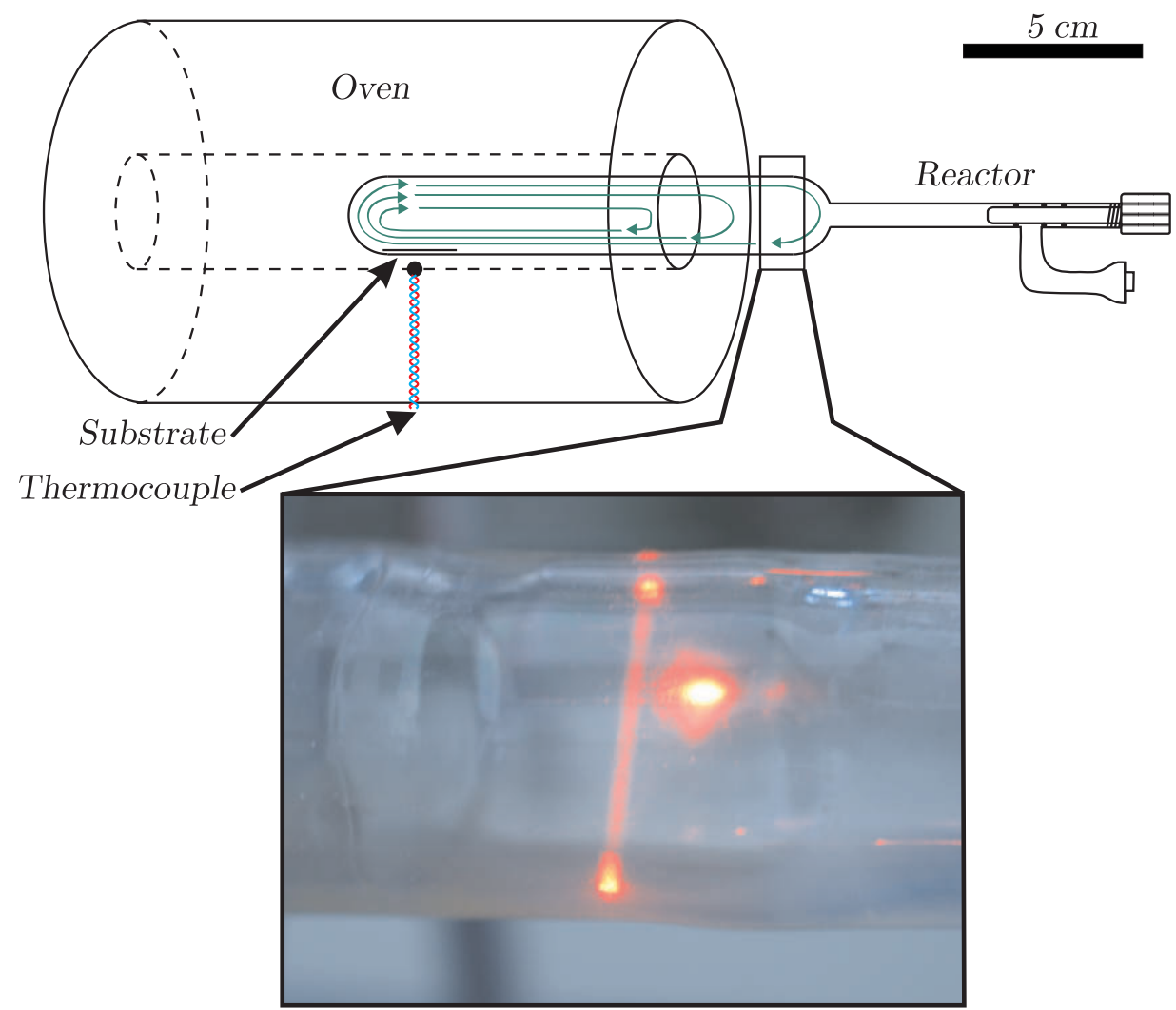

Figure 3.5: Diagram of the CVD setup with the reactor inside the oven. The convection current of the colloids is indicated by green arrows. The convection current is caused by the temperature difference between the hot and cold part of the reactor. The tubular oven has a temperature gradient, being hottest in the center and gradually decreasing in temperature towards the opening. In the figure a photograph of part of the reactor where a laser path is visible during the colloids formation is presented.

5. The convection current will cause that the silicon colloids layer is not uniform throughout the substrate. The highest thickness value for the silicon colloids layer is obtained around the middle of the substrate, when the substrate is located in the center of the oven. Along the temperature gradient of the reactor and oven, the silicon colloids layer thickness will decrease towards the edges of the substrate, forming a smooth natural wedge on both ends as shown in figure 3.6(c). Along the width of the substrate the silicon colloids layer is almost uniform except from the edge where a bulge is formed, as depicted in figure 3.6(b). 


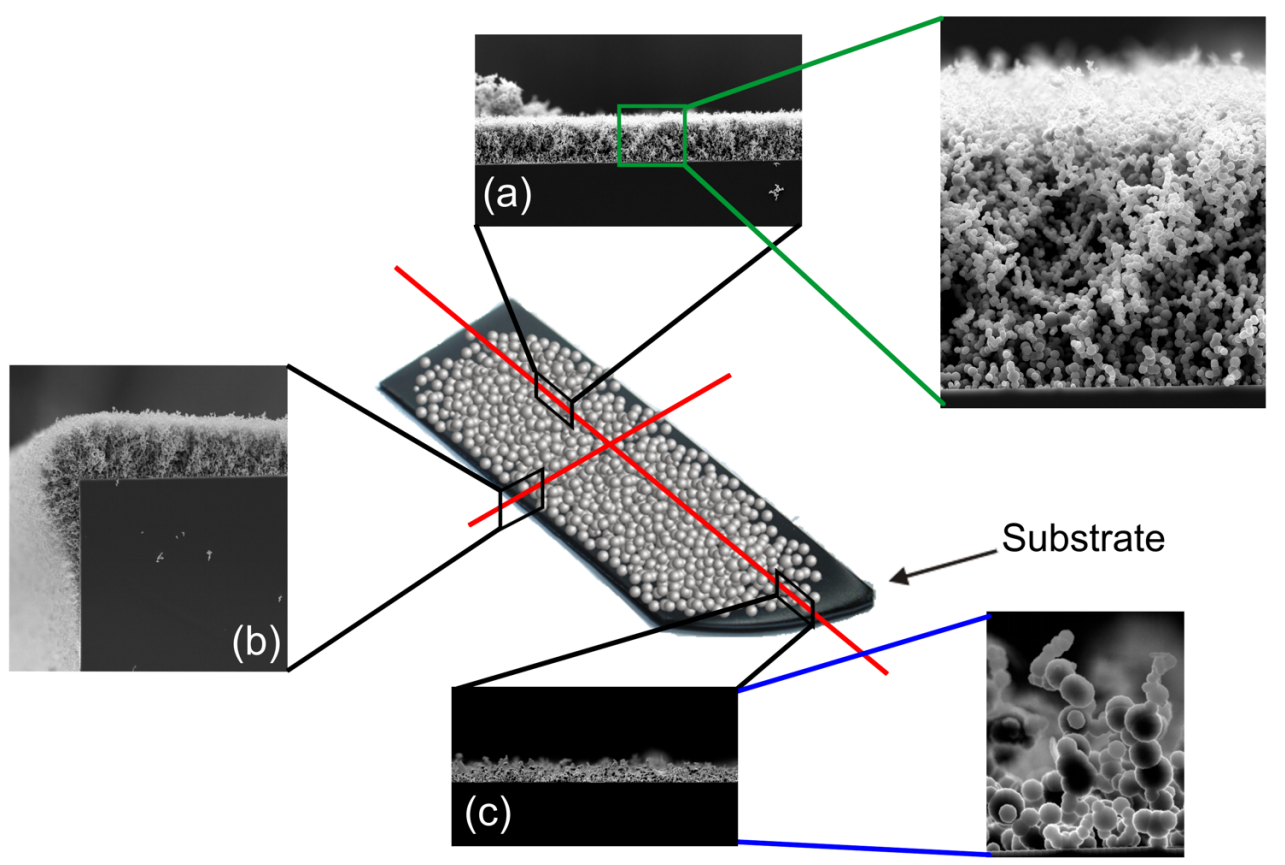

Figure 3.6: Cut through profile of a silicon colloids layer. Along the length of the substrate the highest thickness value is obtained around the middle (where substrate is located in the center of the oven) as indicated by (a). From point (a) to point (c) the thickness decreases gradually. This decrease in thickness will result in a natural wedge on both ends of the substrate. Along the width of the substrate the silicon layer is almost uniform except from the edge where a bulge if formed as indicated by (b).

\subsection{Crystallinity and hydrogen content of silicon colloids}

So far, properties like sphere diameter and thickness of silicon colloids layer have been studied. Another important structural characteristic is that of the type of silicon the colloids are made of. This type can be either amorphous or polycrystalline, depending mainly on the decomposition temperature. At the same time, because the synthesis process is based on the decomposition of a gas (disilane, $\mathrm{Si}_{2} \mathrm{H}_{6}$ ) that contains hydrogen, the obtained silicon will also include hydrogen atoms. This is specially true in the case of amorphous silicon, i.e. this type should be called hydrogenated amorphous silicon.

Generally speaking, amorphous silicon colloids are obtained by using 
decomposition temperatures below $650{ }^{\circ} \mathrm{C}$, while higher temperatures give rise to polycrystalline silicon colloids. Also, amorphous silicon colloids could be converted into polycrystalline ones by applying an annealing treatment at temperatures higher than $650{ }^{\circ} \mathrm{C}$ in vacuum. In any case, no difference in surface roughness and sphericity was observed for the two types of silicon as we will see in section 3.4.

Different techniques were used in order to characterize the crystallinity of silicon colloids: X-Ray powder Diffraction (XRD), Raman scattering and High Resolution Transmission Electron Microscopy (HRTEM). For this purpose three distinct samples, synthesized by using different decomposition parameters were studied. In all of the three cases the decomposition time was 1 hour:

- Sample S1: grown at $400{ }^{\circ} \mathrm{C}$.

- Sample S2: grown at $400{ }^{\circ} \mathrm{C}+$ annealed at $650{ }^{\circ} \mathrm{C}$ during 1 hour in vacuum.

- Sample S3: grown at $800{ }^{\circ} \mathrm{C}$.

No differences in crystallinity were observed in polycrystalline samples obtained via 1 step process, i.e. using a disilane decomposition temperature higher than $650{ }^{\circ} \mathrm{C}$, and via 2 step process, i.e. using a disilane decomposition temperature lower than $650{ }^{\circ} \mathrm{C}$ and performing an annealing treatment at higher temperatures after that, as long as the annealing temperature in the second case coincides with the disilane decomposition temperature in the first case.

The hydrogen content of silicon colloids was studied by using the techniques of mid-infrared spectroscopy and mass spectrometry. In what follows the results obtained from each technique will be described in detail.

\subsubsection{X-Ray powder Diffraction (XRD)}

$\mathrm{XRD}$ is the most usual and easy technique to elucidate the crystalline nature of one material. The peaks of a XRD diagram, their position and their width, indicate the type of material, and the size of the crystal domains the material is made of. Single crystals produce very sharp peaks, that become broader with decreasing the size of the crystals. In a XRD diagram of one material, the breadth of the resulting peaks can be 
related to the mean crystallite size by Scherrer equation [3](The Scherrer equation is limited to nano-scale particles no more than $100 \mathrm{~nm}$ large):

$$
L=\frac{K \lambda}{\beta \cos \theta}
$$

Where:

$\beta$ is the width of the peak at half maximum intensity in radians

$K$ is a constant that varies with the method of taking the breadth $(\mathrm{K}=1)$

$\lambda$ is the wavelength of incident $\mathrm{x}$-rays $(\lambda=1.54187 \AA)$

$\theta$ is the center angle of the peak (Bragg angle)

$L$ is the crystallite length

XRD measurements were realized on layers of silicon colloids deposited on fused silica as the substrate. The substrate does not contribute to the signal, because it is amorphous. The measurements were performed on a XRD system consisting of Philips PW1830 generator, PW1710 diffraction control and PW1820 goniometer, and also on PANalytical X'Pert PRO diffractometer. The X-ray beam is around $4 \mathrm{~mm}$ in diameter. Therefore, it is a macroscopic experiment that records and averages the signal of many colloids at the same time.

The resulting XRD diagrams for samples S1, S2 and S3 are showed in figure 3.7 (a), (b), and (c) 3.7(b) and 3.7(c) respectively. The XRD diagram for a single silicon crystal (silicon wafer powder) is also provided as the reference sample (figure $3.7(\mathrm{~d})$ ).

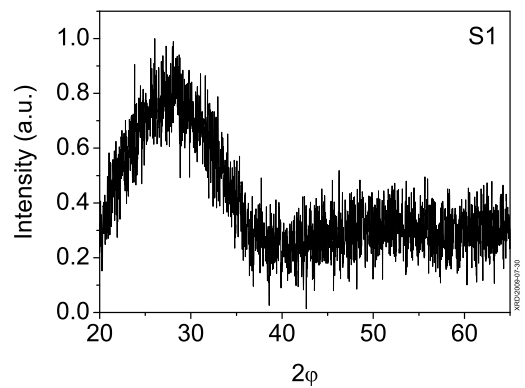

(a)

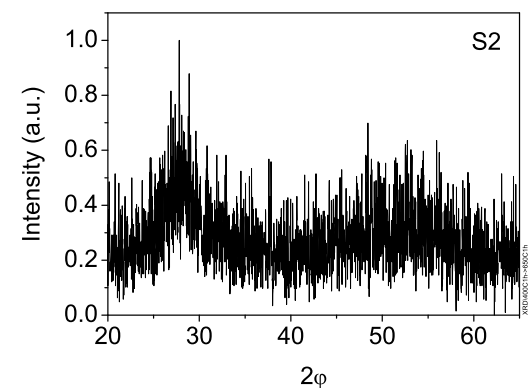

(b)

Figure 3.7: XRD diagrams for silicon colloids grown at (a) $400{ }^{\circ} \mathrm{C}$ (sample S1), (b) $400{ }^{\circ} \mathrm{C}$ and annealed at $650{ }^{\circ} \mathrm{C}$ during 1 hour (sample S2). Figure continue on next page... 


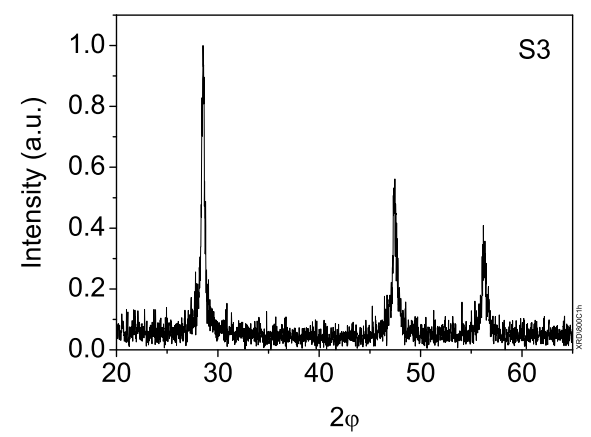

(c)

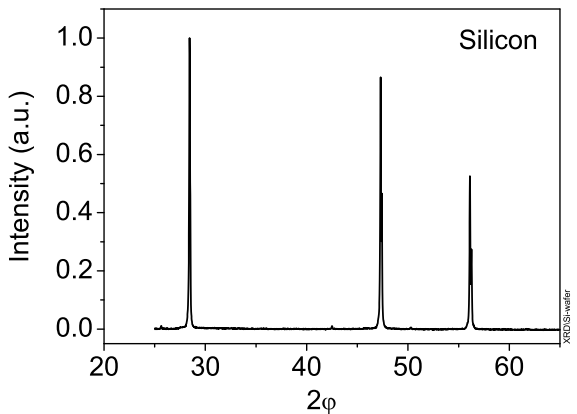

(d)

Continued Figure 3.7: (c) $800{ }^{\circ} \mathrm{C}$ (sample S3), (d) XRD diagram for single crystal silicon powder.

The first observation which can be done is that all of the peaks of the XRD diagram from sample S3 coincide in position with the peaks produced by the single crystal silicon powder (the reference sample), proving that those colloids are in fact made of crystalline silicon. However, their width is slightly larger than the width of the peaks of the silicon powder, due to the limited size of the crystallites composing the colloids. In order to calculate the average crystallite size a Gaussian curve is fit to one of the peaks, namely the strongest one which is placed around $28^{\circ}$. Then, the values obtained for the width of the peak at half maximum intensity (FWHM) and center angle of the peak are used in Scherrer equation. This gives a crystallite length value of $29 \mathrm{~nm}$ (see table 3.1).

\begin{tabular}{|c|c|c|}
\hline Sample & $\beta$ (radians) & Crystallite length \\
\hline S1 & 0.2464 & amorphous \\
S2 & 0.0982 & $2 \mathrm{~nm}$ \\
S3 & 0.0055 & $29 \mathrm{~nm}$ \\
\hline
\end{tabular}

Table 3.1: Crystallite length values calculated using Scherrer Equation.

Samples S1 and S2 do not show so much clear peaks in their XRD diagrams and only the strongest peak positioned around $28^{\circ}$ can be distinguished. In these cases, the application of the same procedure as before gave a much smaller crystallite size: $0.7 \mathrm{~nm}$ for S1, which is considered as amorphous and $2 \mathrm{~nm}$ for $\mathrm{S} 2$ (see table 3.1). The reason why sample S1 gives a much clear peak than sample S2, in spite of the fact 
that $\mathrm{S} 1$ is more amorphous than $\mathrm{S} 2$, is that $\mathrm{S} 1$ was measured by using a apparatus (PANalytical, X'Pert PRO Diffractometer) which provides much better signal to noise ratio than the apparatus used for S2 and S3 (Philips XRD system mentioned above).

\subsubsection{Raman spectroscopy}

While XRD is a macroscopic experiment that allows us to know the average crystalline nature of many colloids, it is also interesting to measure the crystallinity of single colloids. This can be achieved by techniques like micro Raman spectroscopy that will be shown below and HRTEM that will be shown in the next subsection.

The set up for doing micro Raman spectroscopy consists basically of a triple spectrometer (Jobin-Yvon T64000) for filtering undesirable light and recording the Raman signal, a laser for exciting the sample and a microscope by which excitation light can be focused on a single sphere. The excitation source was an Argon (Ar) laser. The line of the laser with wavelength $514.13 \mathrm{~nm}$ was used as this wavelength is absorbed by silicon. The expected Raman stokes shift of silicon (single crystal) is $519 \mathrm{~cm}^{-1}$ [4]. Several authors have studied how the effect of crystallite size influences the Raman shift of semiconductors [5-7].

Generally speaking, the Raman peak of a microcrystalline semiconductor broadens and shifts towards lower energy comparing with that of its single crystal counterpart, due to the relaxation of the momentum conservation rules [5]. Calculations allow correlating the size of the crystallite with the broadening and shift of the Raman peak. Figure 3.8 (a), (b) and (c) show the Raman spectra (Stokes shift) (green lines) for samples S1, S2 and S3, as well as the Raman Stokes shift for single crystal silicon as the reference spectrum (black line). The calculations of Noboru Ohtani and Kazuhiko Kawamura [5] provide a correlation curve between the peak shift $\Delta \omega$ (relative to the peak of single crystal silicon) and the crystallites size. Using that correlation curve, the following values (table 3.2) for the crystallite sizes are obtained:

\begin{tabular}{|c|c|c|}
\hline Sample & Peak shift $\Delta \omega$ & Crystallite diameter \\
\hline S1 & $41 \mathrm{~cm}^{-1}$ & N/A \\
S2 & $2.91 \mathrm{~cm}^{-1}$ & $6 \mathrm{~nm}$ \\
S3 & $1.79 \mathrm{~cm}^{-1}$ & $8 \mathrm{~nm}$ \\
\hline
\end{tabular}

Table 3.2: Peak shift of samples S1, S2 and S3, relative to the peak position of single crystal silicon measured in their Raman spectra, and calculated crystallite diameter. 
The resulting spectrum for sample S1 is a very broad peak, with a very large relative shift. In this case, the relative peak shift is out of the limit of the calculations and it is not possible to estimate the crystallite size, which should be below $3 \mathrm{~nm}$ [5]. This is in good agreement with the previous measurements performed by x-ray diffraction techniques, which indicate that sample S1 is amorphous [8, 9]. The Raman Stokes Shift peaks corresponding to samples S2 and S3 are much less broaden than that of sample S1 and their position is much closer to that of single crystal silicon, thus indicating their crystalline nature. The crystallite size of sample S2 is similar to the value obtained by x-ray measurements, however sample S3 gives a crystal size much smaller (one order of magnitude) than the value provided by x-ray measurements. We have attributed this discrepancy to the large incertitude of the correlation curve at these values.

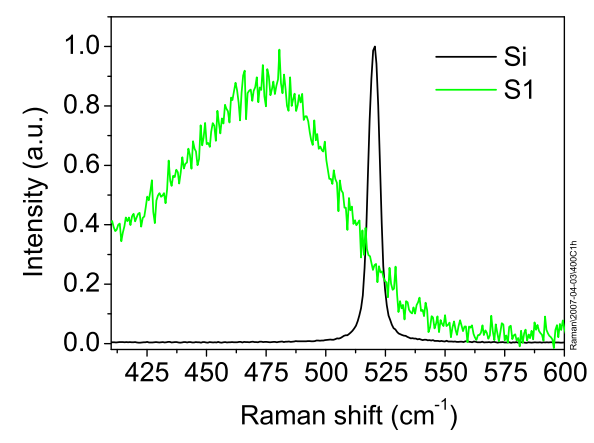

(a)

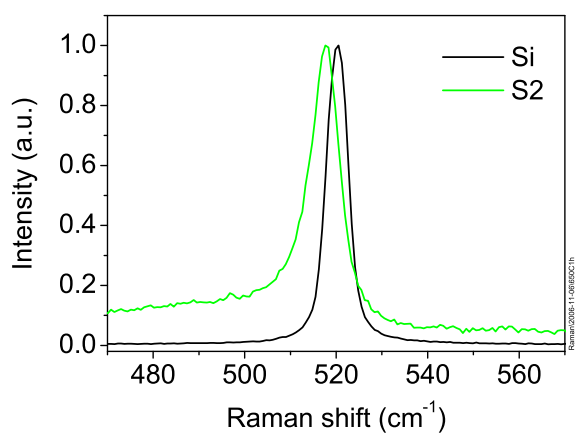

(b)

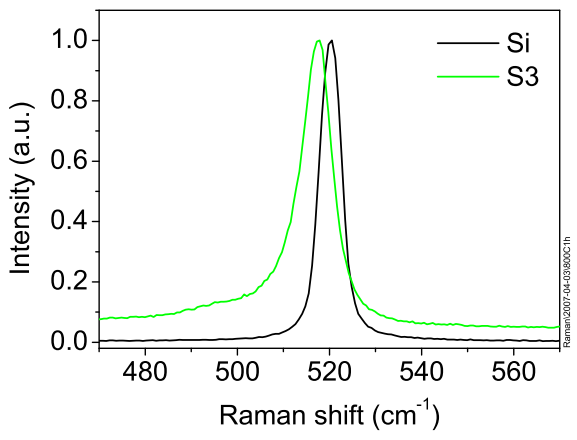

(c)

Figure 3.8: Normalized Raman spectra (in green) for a silicon colloid (a) deposited at $400{ }^{\circ} \mathrm{C}$ for 1 hour (sample S1), (b) deposited at $400 \mathrm{C}$ and annealed at $650{ }^{\circ} \mathrm{C}$ for 1 hour (sample S2) and (c) deposited at $800{ }^{\circ} \mathrm{C}$ for 1 hour (Sample S3). The Raman spectrum of single crystal silicon is also showed in each spectrum (in black) as the reference signal. 


\subsubsection{High Resolution Transmission Electron Mi- croscopy (HRTEM)}

Another technique that can be used to measure the crystallization state of a solid is HRTEM (FEI company, model Tecnai $G^{2}$ ), where the actual packing of atoms can be observed.

Samples for HRTEM were prepared in several steps. The spheres were first embedded in a high temperature epoxy (EPO-TEK 353ND). The cured epoxy with spheres were then polished by hand down to a slice thickness of $300 \mu \mathrm{m}$. The next step was polishing down to a thickness of $30 \mu \mathrm{m}$ on a dimple grinding machine (Fischione model 200 Dimpling Grinder) with diamond paste. The final polishing to obtain an edge with thickness of $30 \mathrm{~nm}$ or less was done by an ion milling machine (Fischione model 1010 Ion Mill) using liquid nitrogen cooling in order to prevent the epoxy from melting.

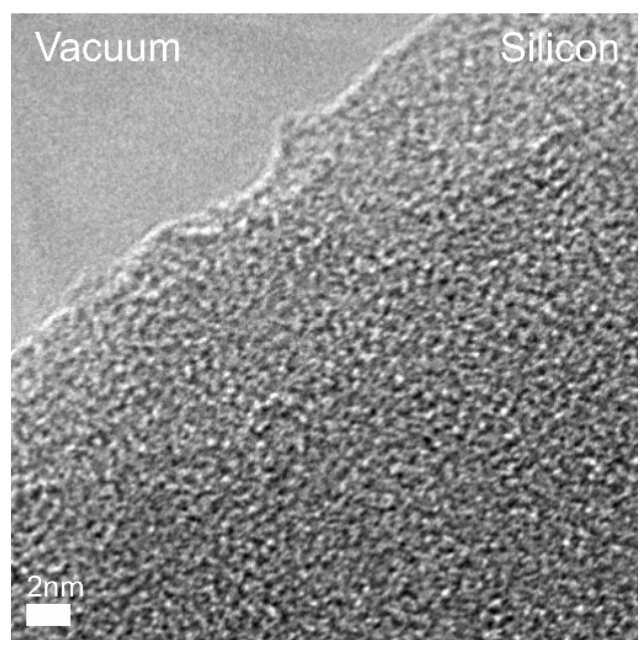

(a)

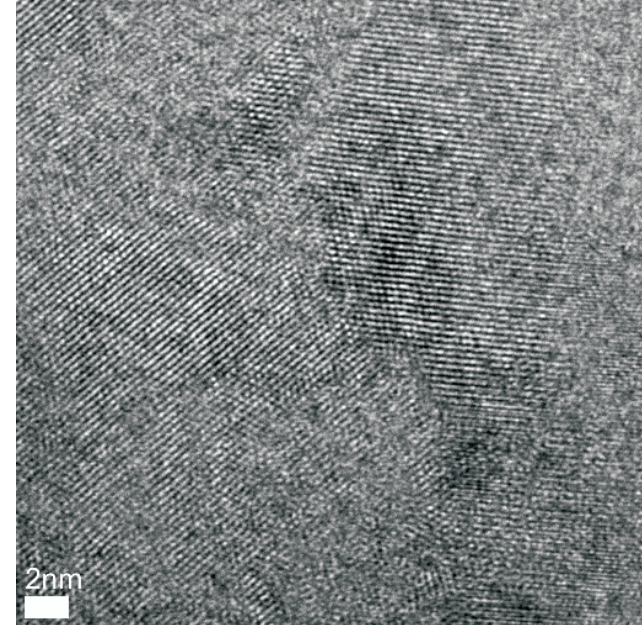

(b)

Figure 3.9: HRTEM images of silicon colloids (a) Sample S1: Silicon colloid deposited at $400{ }^{\circ} \mathrm{C}$ during 1 hour - amorphous silicon, (b) Sample S3: silicon colloid deposited at $800{ }^{\circ} \mathrm{C}$ during 1 hour - polycrystalline silicon. The domains can be clearly seen, having an irregular shape. The size of the crystallites varies from $2 \mathrm{~nm}$ to $60 \mathrm{~nm}$. (c) Electron diffraction pattern of sample S3 illustrating its polycrystalline nature. Figure continue on the next page... 


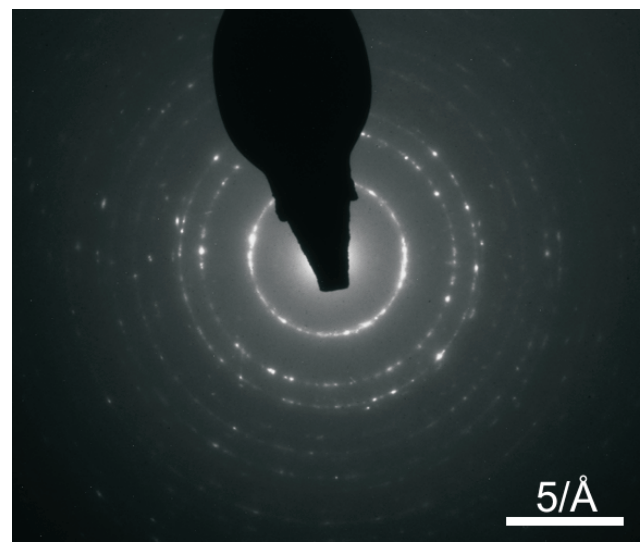

(c)

Continued Figure 3.9: (c) Electron diffraction pattern of sample S3 illustrating its polycrystalline nature.

Two types of samples were studied with HRTEM, S1 and S3. Images in figure 3.9 from HRTEM illustrate that while the spheres corresponding to sample $\mathrm{S} 1$, i. e., deposited at $400{ }^{\circ} \mathrm{C}$ do not show any atomic arrangement, thus confirming their amorphous nature, the spheres of sample S3, i.e., grown at $800{ }^{\circ} \mathrm{C}$ show atomic crystal domains with sizes from $2 \mathrm{~nm}$ to $60 \mathrm{~nm}$. Therefore they are made of poly-crystalline silicon. As the domains are in fact irregular three dimensional grains, the size of the crystallites depends from which side they are observed. The figure 3.9(c) is an electron diffraction pattern of sample S3. The concentric rings confirm the polycrystalline nature of sample S3.

\subsubsection{Hydrogen content of silicon colloids}

As mentioned before, silicon colloids contain some amount of hydrogen atoms due to the synthesis process, specially those colloids grown by using disilane decomposition temperatures below $650{ }^{\circ} \mathrm{C}$. This fact, as well as the removal of hydrogen by an annealing treatment, is studied qualitatively in this section by two techniques: Fourier Transform InfraRed Spectroscopy (FTIR) and Mass Spectrometry.

\subsubsection{Fourier Transform InfraRed Spectroscopy}

A sample of silicon colloids grown by using a disilane decomposition temperature and time of $380{ }^{\circ} \mathrm{C}$ and half an hour respectively was used to 
study the content of hydrogen. The colloids were deposited on a single crystal silicon substrate because silicon is transparent in a wide near, and mid infrared region, and therefore it allows performing infrared transmittance measurements at those wavelengths where molecular bonds are optically active. A Nicolet 710 FTIR spectrometer was used for measuring optical transmittance for wavenumbers from 4000 to $400 \mathrm{~cm}^{-1}$. The light beam averaged the signal of a sample area of $7 \mathrm{~mm}$ in diameter. The silicon colloids sample was measured just after having been synthesized and after an annealing treatment at a temperature of $800{ }^{\circ} \mathrm{C}$ during 1 hour in vacuum. In both cases, the measurements were performed by focusing the spectrometer light spot in the same place. Figure 3.10(a) shows the absorbance spectra for the sample before annealing (in black) and after annealing (in green). Two features of the spectra should be emphasized. They are indicated by two squares in the figure and they have been amplified for a better visualization in figures 3.10(b) and 3.10(c) respectively. Also, the spectra have been baseline corrected in these two figures. The existence of hydrogen in the colloids before the annealing process (black line) is clear when looking at these figures because they show absorbance peaks at $2090 \mathrm{~cm}^{-1}$ and at $630 \mathrm{~cm}^{-1}$ [10-13], which are attributed to stretching of the bonds of $\mathrm{SiH}_{2}$, and bending, rocking and wagging of the bonds of $\mathrm{SiH}, \mathrm{SiH}_{2}$ or $\mathrm{SiH}_{3}$ respectively (see figure 3.11). However, these absorbance peaks disappear after the annealing treatment (green line) and only those peaks associated to $\mathrm{Si}-\mathrm{Si}$ vibration (placed at $611 \mathrm{~cm}^{-1}[14]$ ) and to the native silica (placed at $620 \mathrm{~cm}^{-1}[15]$ ), which grows naturally on silicon after the exposure of the sample to the open air, are still present. Figure 3.10(c) shows also (in red) the optical transmittance of a single crystal silicon wafer (shifted a bit downwards for an easier visualization). This spectrum is very similar to that of the annealed silicon colloids and confirms the removal of the hydrogen by the annealing treatment. 


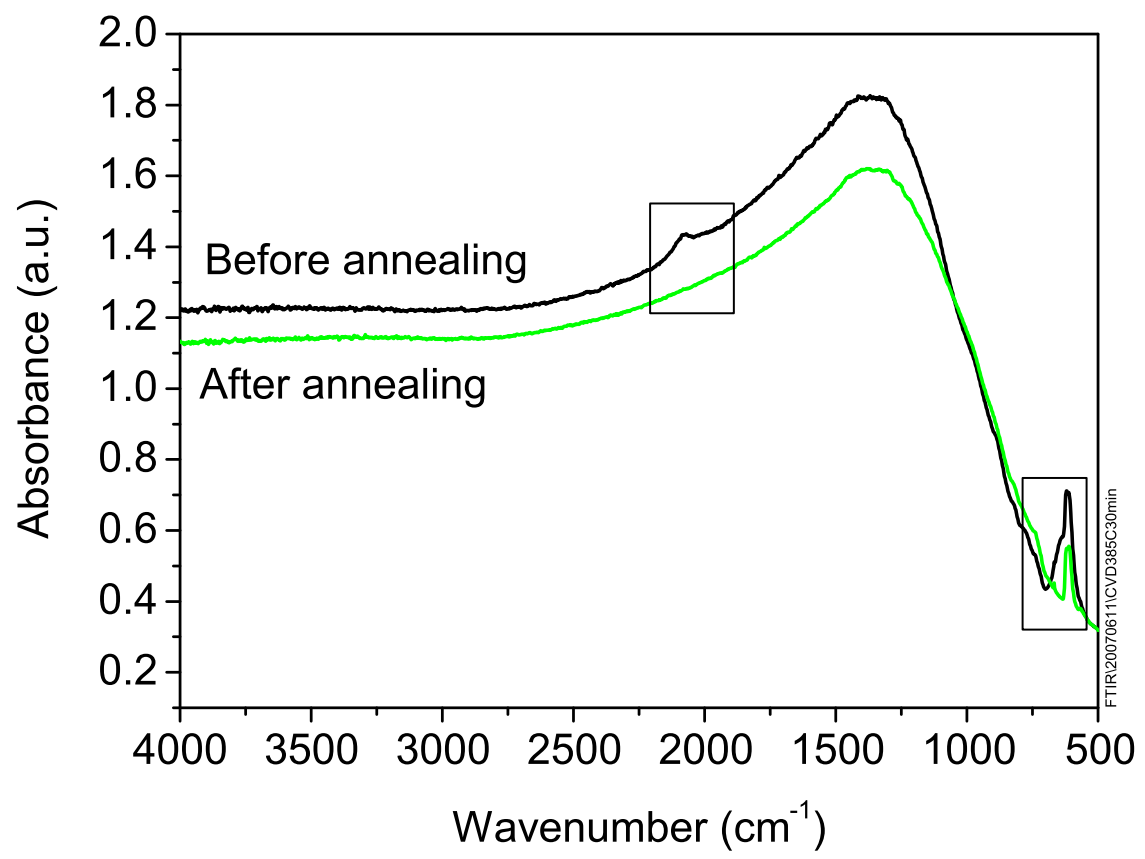

(a)

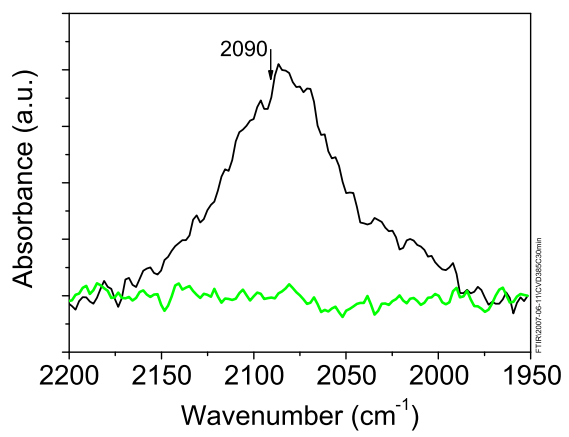

(b)

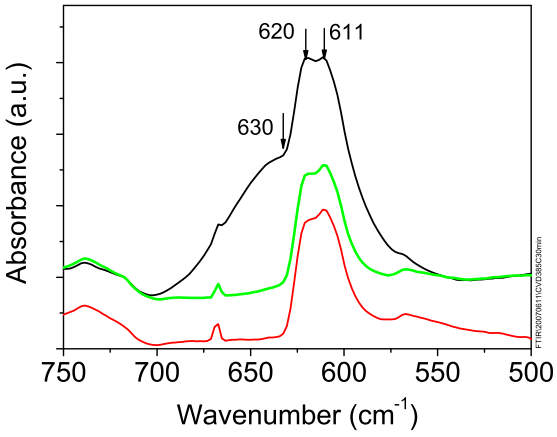

(c)

Figure 3.10: (a) FTIR spectra demonstrating the effect of an annealing treatment at $800{ }^{\circ} \mathrm{C}$ during 1 hour (in green) on a silicon colloids sample grown at $385{ }^{\circ} \mathrm{C}$ for 30 minutes ( in black ). Two regions of the spectra indicated by squares have been amplified and baseline corrected in (b) and (c). They show absorbance peaks associated to $\mathrm{Si}-\mathrm{H}$ vibrations occurring at $2090 \mathrm{~cm}^{-1}$ (b) and $630 \mathrm{~cm}^{-1}$ (c). The red curve in (c) corresponds to a reference sample consisting of a silicon wafer which shows features at $620 \mathrm{~cm}^{-1}$ and at $611 \mathrm{~cm}^{-1}$, associated to Si-Si vibrations and the native silica respectively. This curve has been vertically displaced in order to ease the comparison. 


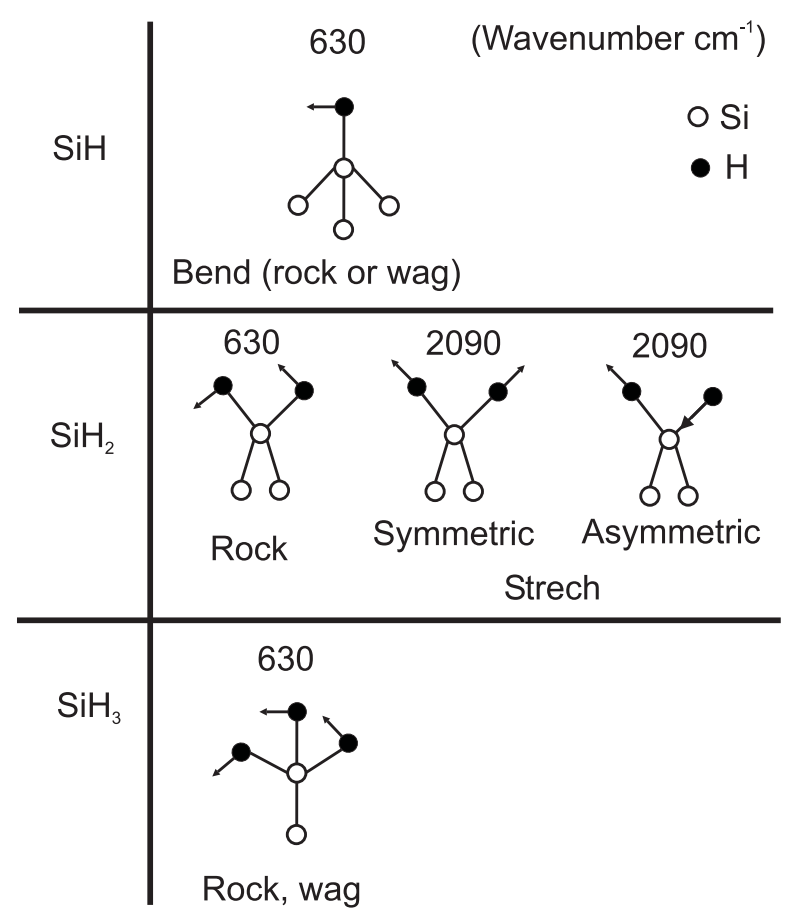

Figure 3.11: Set of $\mathrm{Si}-\mathrm{H}$ vibrational modes for $\mathrm{SiH}, \mathrm{SiH}_{2}$ or $\mathrm{SiH}_{3}$ groups, with frequencies indicated (R. A. Street et al. 1991).

\subsubsection{Mass Spectrometry}

Mass spectrometry allows studying at which temperature hydrogen starts desorbing from amorphous silicon colloids. For this purpose, $50 \mathrm{mg}$ of colloids, that had been synthesized at a disilane decomposition temperature of $550{ }^{\circ} \mathrm{C}$ during 1 hour, were heated progressively from room temperature to $900{ }^{\circ} \mathrm{C}$. During the heating process the desorbed gases were analyzed by a mass spectrometer (Blazers Instruments, Omnistar). Helium with a purity of $99.999 \%$ was used as the bearer gas. Detection of water, atomic oxygen and $\mathrm{OH}$ is related to the presence of water in the bearer gas - Helium.

Figure 3.12 shows the ion current signal provided by the mass spectrometer associated to different detected atom masses as shown in table 3.3 .

While in general all of the curves show a behaviour consisting of a decrease of the ion current as the temperature increases, the ion current associated to $\mathrm{H}_{2}$ increases progressively from $650{ }^{\circ} \mathrm{C}$ on, indicating the 
hydrogen desorption and therefore crystallization starts at this temperature. This result is consistent with the literature data [16, 17], and it is in good agreement with the results obtained by FTIR.

\begin{tabular}{|c|c|}
\hline Atom mass & Description \\
\hline 1 & Atomic hydrogen \\
2 & Hydrogen gas \\
16 & Atomic oxygen \\
17 & OH radical \\
18 & Water $\mathrm{H}_{2} \mathrm{O}$ \\
\hline
\end{tabular}

Table 3.3: Atom masses detected by mass spectroscopy and their associated elements and compounds.

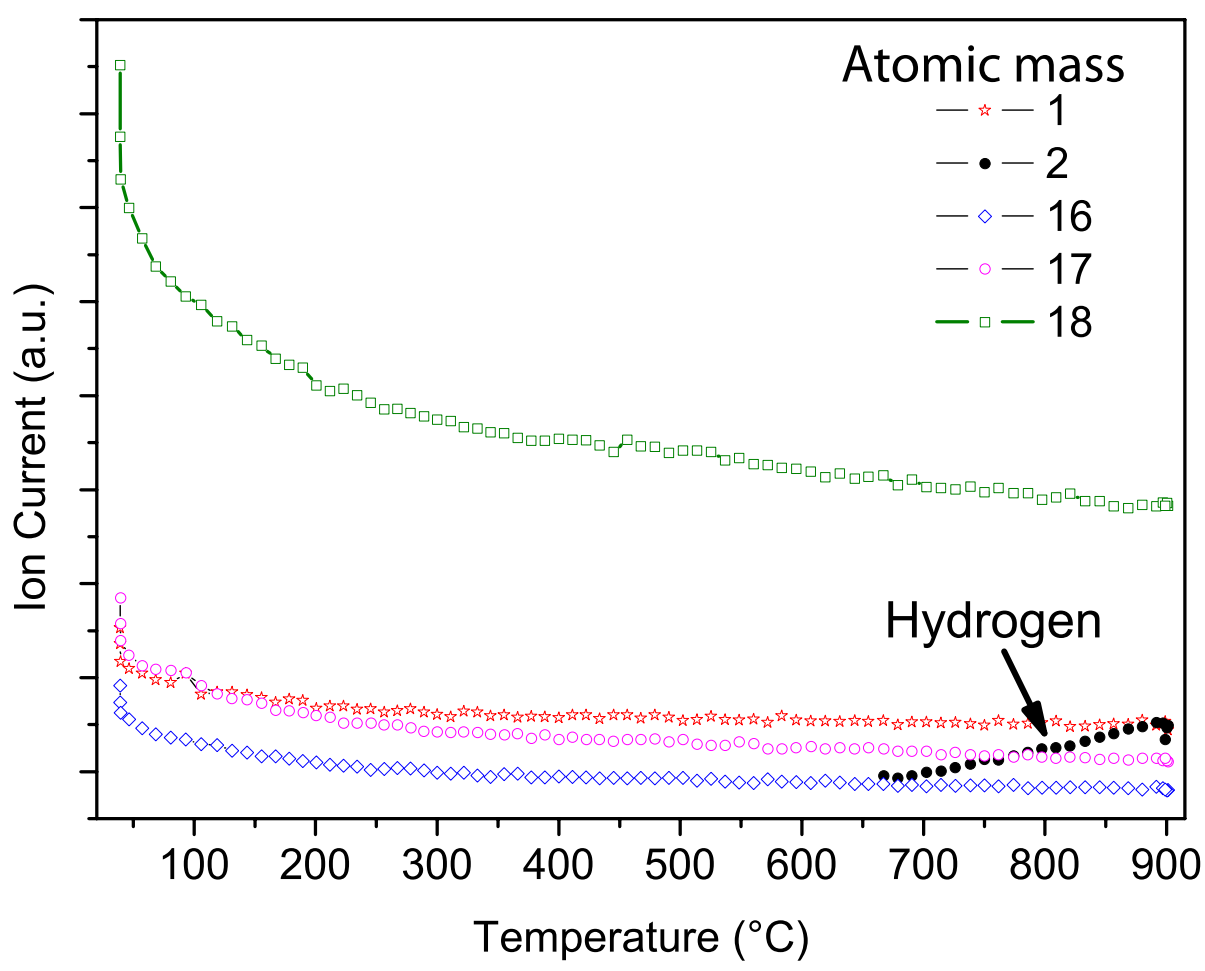

Figure 3.12: Mass spectroscopy of silicon colloids grown at $550{ }^{\circ} \mathrm{C}$ for 1 hour. The spectroscopy is performed while the colloids are heated from room temperature to $900{ }^{\circ} \mathrm{C}$. Emission of hydrogen gas starts when the temperature reaches $660{ }^{\circ} \mathrm{C}$.

In conclusion, the mass spectroscopy confirmed the hydrogenated nature of the amorphous silicon colloids and indicated the temperature of 
$650{ }^{\circ} \mathrm{C}$ as the one at which hydrogen starts desorbing from them.

\subsection{Surface morphology}

The surface of silicon colloids appears perfectly smooth when they are observed by Scanning Electron Microscopy (SEM) as depicted in figure 3.13 although they show some defects, for instance the one indicated by an arrow, which are due to the fact that they were in contact to each other during the synthesis process, as we mentioned in section 2.2. The microscope used here has a resolution of $30 \mathrm{~nm}$. In order to better verify the surface smoothness of the microspheres, Atomic Force Microscope AFM (Veeco Metrology Group, MultiMode Scanning Probe Microscope with The NanoScope 3D Controller) measurements were performed on to the colloids surface. This technique is very appropriate for surface roughness measurements and it provides a high resolution, of the order of $1 \AA$.

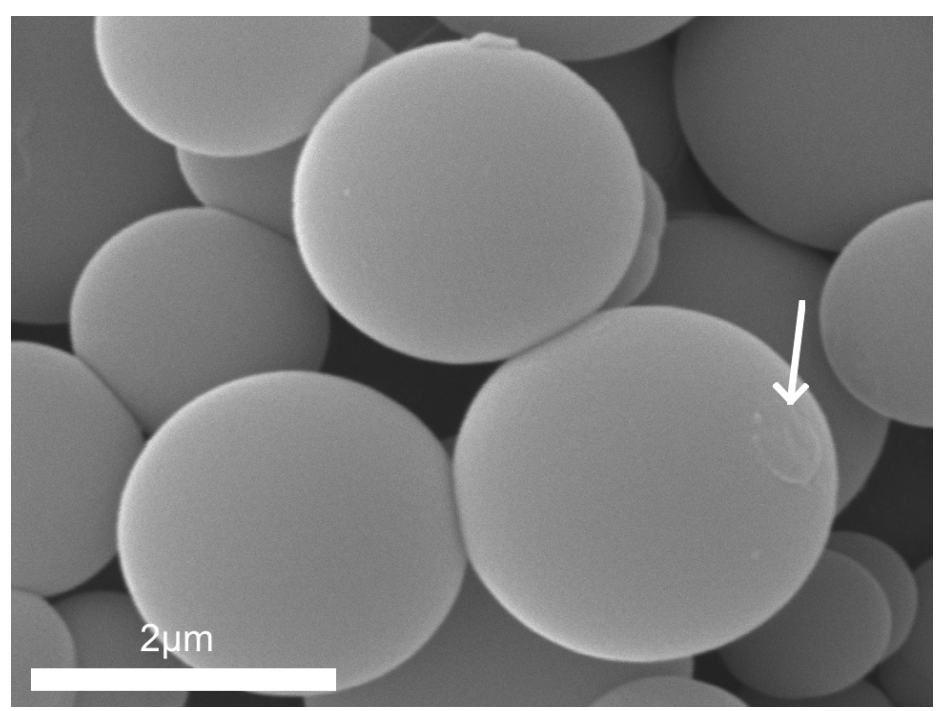

Figure 3.13: Silicon colloids analyzed by SEM. The surface of the colloids appears very smooth and no features can be distinguished except some defects associated to the contact points between them (see for instance the one indicated by an arrow).

Figure 3.14(a) shows a three dimensional image obtained by AFM in tapping mode of the surface of a silicon colloid that was synthesized at $550{ }^{\circ} \mathrm{C}$ for 1 hour and annealed in vacuum at $800{ }^{\circ} \mathrm{C}$ during 1 hour, i.e. 
it is a polycrystalline silicon colloid. The surface appears very smooth (the diagonal ridges are an artefact from the AFM scanning procedure). It has a mean roughness of $0.3 \mathrm{~nm}$. This roughness $R_{a}$ was calculated by flattening the image (figure 3.14(b)) and averaging the $\mathrm{Z}$ values as follows:

$$
R_{a}=\frac{1}{n} \sum_{j=1}^{n}\left|Z_{j}\right|
$$

Flattening is a procedure where the curvature is removed by a second grade polynomial. Several spheres synthesized by using different decomposition parameters, made of amorphous and poly-crystalline silicon were analyzed by AFM. In general roughness values from $0.2 \mathrm{~nm}$ to $0.6 \mathrm{~nm}$ for regions of $80000 \mathrm{~nm}^{2}$ were obtained. In optics a surface is considered flat when the roughness of the surface is below $\lambda / 20$, therefore the optical quality of the spheres can be considered excellent when used for wavelengths above $20 \mathrm{~nm}$.

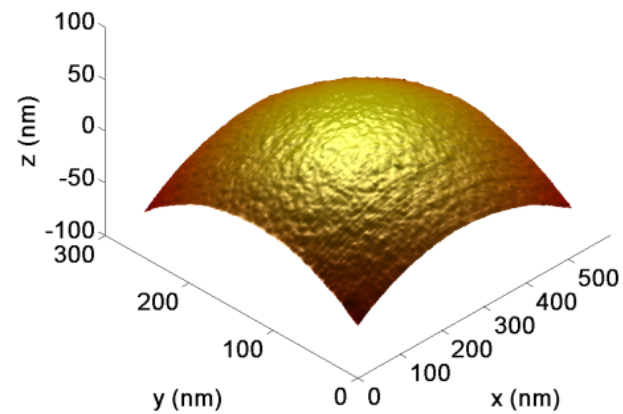

(a)

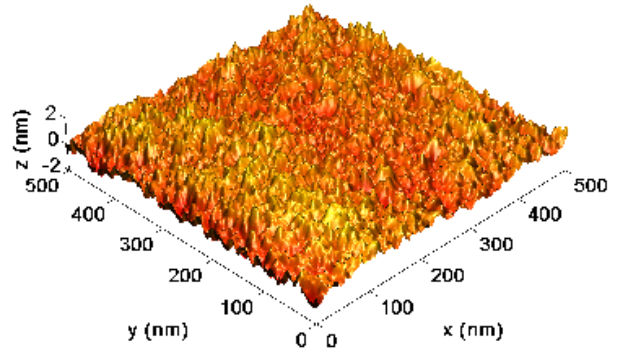

(b)

Figure 3.14: (a) Colloid surface analyzed by AFM. The silicon colloid was manufactured at $550{ }^{\circ} \mathrm{C}$ for 1 hour and recrystallized in vacuum at $800{ }^{\circ} \mathrm{C}$ during 1 hour. A $500 \mathrm{~nm}$ by $500 \mathrm{~nm}$ region is scanned. The diagonal ridges are an artefact from the AFM scanning procedure. (b) The image of (a) processed by flattening. Note that $\mathrm{Z}$ axis is not to scale with $\mathrm{X}$ and $\mathrm{Y}$ axis. The $\mathrm{Z}$ axis is exaggerated 25 times in order to reveal more features of the surface.

\subsection{Conclusions}

In this chapter we have analyzed and described the structural properties of silicon colloids. These properties are: silicon colloid sphere diameter distribution, crystallinity, hydrogen content, and surface morphology. 
The main conclusions of the chapter are summarized in the following points:

- Silicon colloids, grown by using disilane decomposition temperatures from $400{ }^{\circ} \mathrm{C}$ to $800{ }^{\circ} \mathrm{C}$, are poly-disperse in size. They have a diameter from 0.5 to $3.5 \mu \mathrm{m}$.

- The size distribution of the colloids is not symmetric but biased towards larger sphere diameters. The mode diameter of the distributions is from 0.94 to $1.66 \mu \mathrm{m}$, and the temperature at which the mode is largest is around $600{ }^{\circ} \mathrm{C}$.

- Silicon colloids can be amorphous or polycrystalline depending mainly on the disilane decomposition temperature utilized in the synthesis process. While temperatures below $650{ }^{\circ} \mathrm{C}$ yield amorphous silicon colloids that contain hydrogen, higher temperatures produce polycrystalline ones. Also, the hydrogen of the amorphous silicon colloids can be removed and they become polycrystalline ones by an annealing process in vacuum to a temperature higher than $650{ }^{\circ} \mathrm{C}$. In any case, there is no difference in crystallinity between polycrystalline colloids obtained directly in the synthesis process and those obtained by an annealing process from amorphous ones as long as the disilane decomposition temperature and the annealing temperature are the same.

- The crystallite size of polycrystalline colloids depends on the disilane decomposition temperature utilized during their synthesis process and on the annealing temperature in case of the polycrystalline colloids that come from annealing amorphous ones. The crystallite size increases from $2 \mathrm{~nm}$ to $30 \mathrm{~nm}$ as the temperature increases from $650{ }^{\circ} \mathrm{C}$ to $800{ }^{\circ} \mathrm{C}$.

- The surface roughness of silicon colloids is from 0.2 to $0.6 \mathrm{~nm}$, what makes them optically flat for wavelengths larger than $20 \mathrm{~nm}$. No surface roughness difference was observed between amorphous silicon colloids and polycrystalline ones. 


\section{Bibliography}

[1] Carolyn Kimme, Dana Ballard, and Jack Sklansky. "Finding circles by an array of accumulators". Communications of the ACM, 18(2):120-122, 1975.

[2] D. H. Ballard. "Generalizing the hough transform to detect arbitrary shapes". Pattern Recognition, 13(2):111-122, 1981.

[3] A. L. Patterson. "The Scherrer Formula for X-Ray Particle Size Determination". Phys. Rev., 56(10):978-982, Nov 1939.

[4] Paul A. Temple and C. E. Hathaway. "Multiphonon Raman Spectrum of Silicon". Phys. Rev. B, 7(8):3685-3697, Apr 1973.

[5] Noboru Ohtani and Kazuhiko Kawamura. "Theoretical investigation of Raman scattering from microcrystallites". Solid State Communications, 75(9):711 - 715, 1990.

[6] I. H. Campbell and P. M. Fauchet. "The effects of microcrystal size and shape on the one phonon Raman spectra of crystalline semiconductors". Solid State Communications, 58(10):739 - 741, 1986.

[7] H. Richter, Z. P. Wang, and L. Ley. "The one phonon Raman spectrum in microcrystalline silicon". Solid State Communications, 39(5):625 - 629, 1981.

[8] Z Iqbal and S Veprek. "Raman scattering from hydrogenated microcrystalline and amorphous silicon". Journal of Physics C: Solid State Physics, 15(2):377, 1982.

[9] Mile Ivanda, Ozren Gamulin, and Wolfgang Kiefer. "Mechanism of Raman scattering in amorphous silicon". Journal of Molecular Structure, 480-481:651 - 655, 1999.

[10] R. A. Street. Hydrogenated amorphous silicon. Cambridge University Press, The Edinburgh Building, Cambridge CB2 2RU, UK, 1991.

[11] E. C. Freeman and William Paul. "Infrared vibrational spectra of RF-sputtered hydrogenated amorphous silicon". Phys. Rev. B, 18(8):4288-4300, Oct 1978. 
[12] Y. H. Ogata, T. Tsuboi, T. Sakka, and S. Naito. "Oxidation of Porous Silicon in Dry and Wet Environments under Mild Temperature Conditions". Journal of Porous Materials, 7(1):63-66, 2000.

[13] M. H. Brodsky, Manuel Cardona, and J. J. Cuomo. "Infrared and Raman spectra of the silicon-hydrogen bonds in amorphous silicon prepared by glow discharge and sputtering". Phys. Rev. B, 16(8):3556-3571, Oct 1977.

[14] William C. O'Mara, Robert B. Herring, and Lee P. Hunt. Handbook of semiconductor silicon technology. Noyes publication, Park Ridge, New Jersey, US, 1990.

[15] Beata Sweryda-Krawiec, Thierry Cassagneau, and Janos H. Fendler. "Surface Modification of Silicon Nanocrystallites by Alcohols". The Journal of Physical Chemistry B, 103(44):9524-9529, 1999.

[16] P. K. Lim, W. K. Tam, L. F. Yeung, and Lam F. M. "Effect of hydrogen on dangling bond in a-Si thin film". Journal of Physics: Conference Series, 61(1):708-712, 2007.

[17] P. K. Lim and W. K. Tam. "Local Vibrational Modes And The Optical Absorption Tail Of Amorphous Silicon". International Journal of Modern Physics B, 20(25/27):4261 - 4266, 2006. 


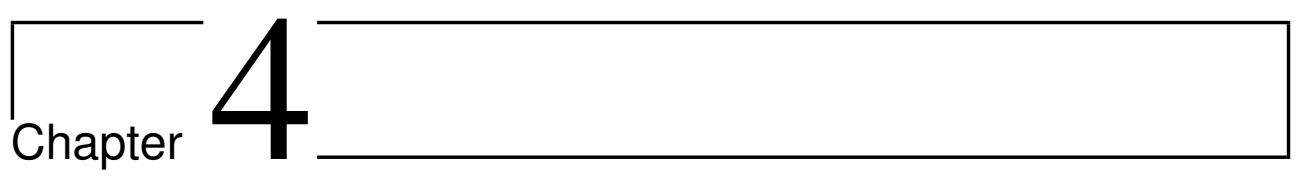

\section{Optical properties of silicon colloids}

\subsection{Introduction}

In this chapter we will see how the structural characteristics of silicon colloids that were studied in chapter 3 , influence their optical properties. Particularly, it should be emphasized the fact that the colloidal geometry approaches quite a lot to that of a perfect sphere with a very smooth surface. This is the key factor for achieving a very good fitting of the experimental optical transmittance to that given by Mie theory in a wide spectral range, by using the diameter of the sphere as the only fitting parameter. Also, we will see how an apparently not so important structural property like the sphere size distribution of a photonic sponge influences notably its optical transmittance. Optical properties were measured by using different setups. Each setup is explained in its corresponding section before the obtained results. For measuring the properties of single colloids and photonic sponges, it would have been ideal to have more apparatus like lasers, detectors and spectrometers covering a wide spectral range, from near to mid-far infrared. That was not the case and only some parts of their optical properties were able to be studied. Nevertheless, this is a good starting point for a new material, that reveals at the same time a huge amount of studies and experiments that can be realized from now on. The chapter has been organized as follows: The first section consists of a theoretical introduction to the light scattering and optical trapping by particles phenomena. After that, the results of optical properties of silicon colloids, measured one by one, are detailed. Then the results corresponding to photonic sponges are presented. Here 
two aspects have been analyzed: the influence of the sphere size distribution and the influence of the colloidal filling fraction. Finally we give the conclusions of the chapter.

\subsection{Theory about light scattering by par- ticles}

\subsubsection{Introduction}

The simplest case when analyzing the scattering of light concerns a single spherical particle. Mie theory (also called Lorenz-Mie-Debye theory) describes the scattering of electromagnetic radiation by spherical particles when their size is of the same order of magnitude as the radiation wavelength. On the other side, Rayleigh scattering theory assumes the incident light wavelength is much larger than the particle size. Mie theory explains for example the opaque white appearance of milk and other disordered substances. Milk is a suspension of many small fat droplets with a diameter of $4 \mu \mathrm{m}$ across, each with a strong tendency to scatter light: when a light wave hits a fat droplet its direction of travel will be changed in an arbitrary way. A light wave passing through a glass of milk will be scattered thousands of times by thousands of fat droplets. Rayleigh scattering can explain the blue color of the sky, because in this regime light with higher frequency (blue light) is scattered by the molecules composing the air more efficiently than light with low frequency (red light).

If light impinges on a particle of an arbitrary shape (as shown in figure 4.1) it will be scattered along all directions of space. Both scattered and absorbed light will attenuate the incident beam. In this thesis we have assumed zero absorption and elastic scattering, i.e. the scattered light has the same frequency or wavelength that the incident light. This is so, because our measurements are performed in the near infrared range where silicon is transparent, and the light beams we used have not enough energy to provoke non-linear phenomena. 


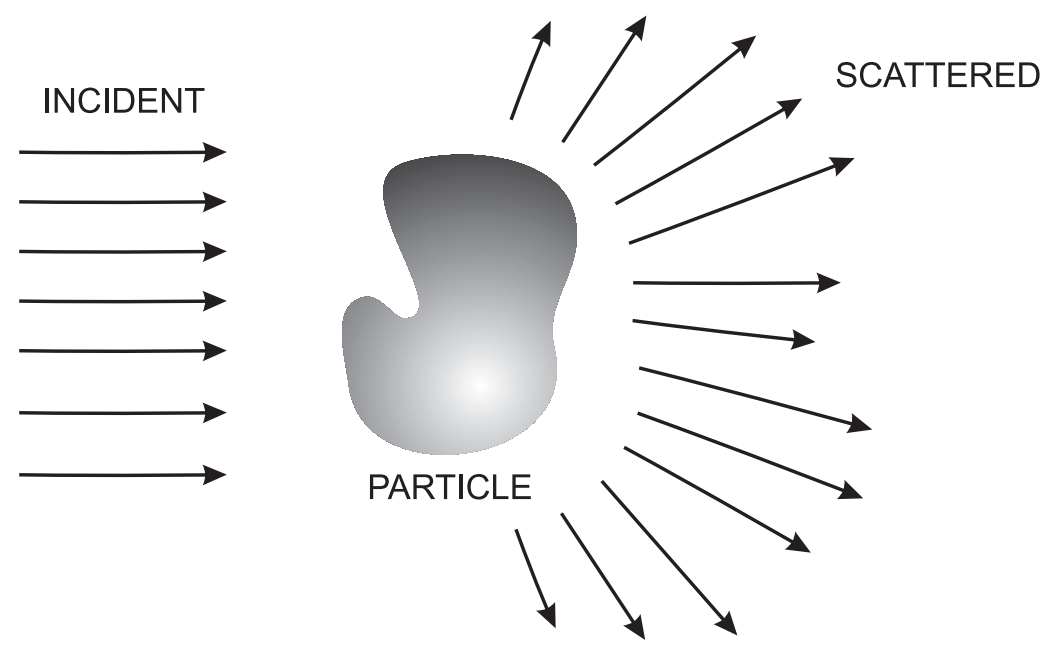

Figure 4.1: Scattering of light by a particle. A incident wave from left impinges on a particle. The particle can both absorb and scatter light in all directions.

In the next sections of this brief theoretical introduction to the problem of optical scattering by particles we will concentrate mainly on that regime where the wavelength of light is of the same order of magnitude as the size of the particle, i.e. in the Mie scattering regime. As we will see, in spite of the complexity of the problem, an analytical solution can be found when the particle consists of a sphere.

\subsubsection{The light trapping phenomenon}

One of the most astonishing optical property of a spherical object whose refractive index is higher than the refractive index of the surrounding environment is the fact that it can trap light. This is a resonance phenomenon that occurs at some particular wavelengths, producing what it is called resonance modes. But, what does "an object traps light" mean?. It means that, for instance a short pulse of light of say 10 picoseconds of duration, will be captured by the spherical object and remain inside it for some time, for example 1 nanosecond. It does not mean that the captured light will be released suddenly after that time but it means that light will be lost, exponentially decaying during this time. In this context, there is a parameter, the quality factor $Q$, that indicates the energy stored in a cavity. It is defined as the energy stored in the cavity divided by the energy loss per cycle [1]. $Q$ depends of course on the resonant mode. The same cavity can support modes of very different $Q$ 's. The 
higher the $Q$ the more energy is stored in the cavity. This parameter can also be calculated from the spectral signal produced by the cavity in the frequency domain as $\frac{\lambda_{0}}{\Delta \lambda}$, where $\lambda_{0}$ is the resonant cavity wavelength and $\Delta \lambda$ is the linewidth (Full Width at Half Maximum, FWHM) of the cavity mode. Therefore thinner peaks in the spectra are associated to higher Q's.

The basic phenomenon underlying the trapping effect in spherical cavities is the total internal reflection of light. Because of this phenomenon, light is captured by the object tangentially and it is reflected in its inside wall several times, (see figure 4.2), "drawing" a particular pattern of electric field intensity distribution which depends on the particular resonance mode. Because of their resemblance to the resonant modes of acoustic waves in circular cavities, these modes are also called Whispering Gallery Modes or WGM after being first noticed by Lord Rayleigh [2].

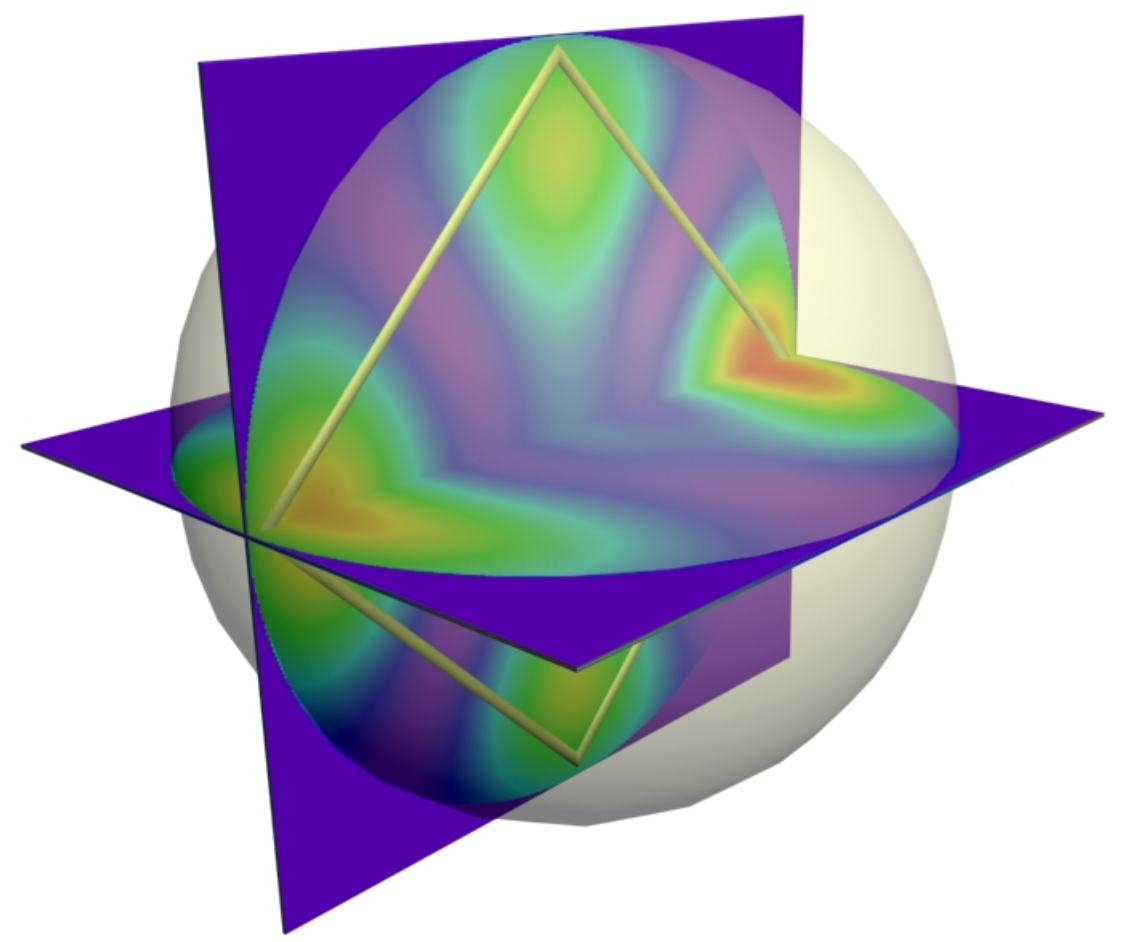

Figure 4.2: Electric field intensity distribution of the resonant mode $\mathrm{TE}_{2,1}$ inside a silicon sphere $(n=3.5)$. The yellow bar indicate the resonance plane. 


\subsubsection{The Mie theory}

There is a theory, developed by Mie [3] in 1908, that gives an analytical solution of the light resonance phenomena in a sphere. This theory can be found in several books, like that of Bohren and Huffman [4], Stratton [5], Hulst [6], Born and Wolf [7], etc. Here, we will summarize the most important points of the theory. Basically it consists of solving the Maxwell equations for an electromagnetic field that interacts with a sphere. For this purpose, the fields are developed in infinite series of functions with spherical symmetry. Figure 4.3 illustrates the spherical coordinates used for this development.

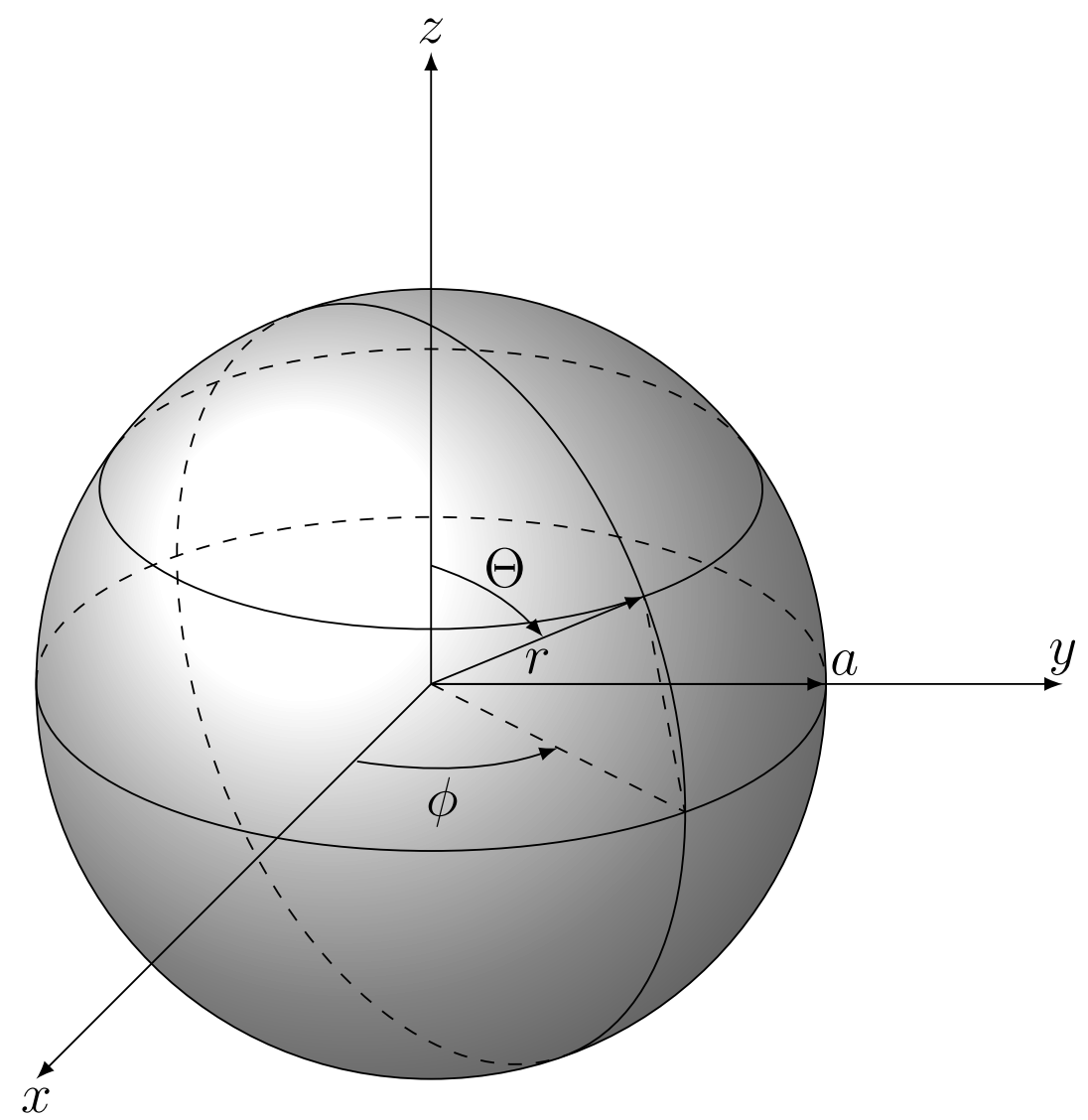

Figure 4.3: Spherical coordinate system. A point in the spherical coordinates is described by three coordinates $(r, \theta, \phi)$, where $r$ represents the radial distance of a point from a fixed origin, $\theta$ represents the zenith angle from the positive z-axis and $\phi$ represents the azimuth angle from the positive $\mathrm{x}$-axis. The sphere of this illustration has a radius $a$. 
This way, the scattered electric $\mathbf{E}_{s}$ and magnetic $\mathbf{H}_{s}$ fields can be written, after several manipulations, as follows (for more details see [4]):

$$
\begin{array}{r}
\mathbf{E}_{s}=\sum_{n=1}^{\infty} E_{n}\left(i a_{n} \mathbf{N}_{e 1 n}^{(3)}-b_{n} \mathbf{M}_{o 1 n}^{(3)}\right), \\
\mathbf{H}_{s}=\frac{k}{\omega \mu} \sum_{n=1}^{\infty} E_{n}\left(a_{n} \mathbf{M}_{e 1 n}^{(3)}+i b_{n} \mathbf{N}_{o 1 n}^{(3)}\right) .
\end{array}
$$

where

$$
E_{n}=i^{n} E_{0} \frac{(2 n+1)}{n(n+1)} .
$$

The $k$ is the propagation constant $\left(k=\frac{2 \pi}{\lambda}\right), \omega$ the angular frequency and $\mu$ is the permeability of the medium, $E_{0}$ is the incident electric field amplitude and $i$ is the imaginary unit. The superscript "(3)" of $\mathbf{N}$ and $\mathbf{M}$ indicates the type of Bessel function $z_{n}(\rho)$, in this case it is $h_{n}^{(1)}$ (see equation (4.7) for definition).

$\mathbf{M}_{o 1 n}, \mathbf{M}_{e 1 n}, \mathbf{N}_{o 1 n}$ and $\mathbf{N}_{e 1 n}$ are defined as follows:

$$
\begin{aligned}
\mathbf{M}_{o 1 n} & =\cos \phi \pi_{n}(\cos \theta) z_{n}(\rho) \hat{\mathbf{e}}_{\theta}-\sin \phi \tau_{n}(\cos \theta) z_{n}(\rho) \hat{\mathbf{e}}_{\phi}, \\
\mathbf{M}_{e 1 n} & =-\sin \phi \pi_{n}(\cos \theta) z_{n}(\rho) \hat{\mathbf{e}}_{\theta}-\cos \phi \tau_{n}(\cos \theta) z_{n}(\rho) \hat{\mathbf{e}}_{\phi}, \\
\mathbf{N}_{o 1 n} & =\sin \phi n(n+1) \sin \theta \pi_{n}(\cos \theta) \frac{z_{n}(\rho)}{\rho} \hat{\mathbf{e}}_{r} \\
& +\sin \phi \tau_{n}(\cos \theta) \frac{\left[\rho z_{n}(\rho)\right]^{\prime}}{\rho} \hat{\mathbf{e}}_{\theta}+\cos \phi \pi_{n}(\cos \theta) \frac{\left[\rho z_{n}(\rho)\right]^{\prime}}{\rho} \hat{\mathbf{e}}_{\phi}, \\
\mathbf{N}_{e 1 n} & =\cos \phi n(n+1)(\sin \theta) \pi_{n}(\cos \theta) \frac{z_{n}(\rho)}{\rho} \hat{\mathbf{e}}_{r} \\
& +\cos \phi \tau_{n}(\cos \theta) \frac{\left[\rho z_{n}(\rho)\right]^{\prime}}{\rho} \hat{\mathbf{e}}_{\theta}-\sin \phi \pi_{n}(\cos \theta) \frac{\left[\rho z_{n}(\rho)\right]^{\prime}}{\rho} \hat{\mathbf{e}}_{\phi} .
\end{aligned}
$$

where $\pi_{n}$ and $\tau_{n}$ are defined as

$$
\pi_{n}=\frac{P_{n}^{1}}{\sin \theta}, \quad \tau_{n}=\frac{d P_{n}^{1}}{d \theta} .
$$


where $P_{n}^{1}$ is the Legendre functions of the first kind, and $\rho=k r$ is a dimensionless variable. The coefficients $a_{n}$ and $b_{n}$ of equation (4.1) can be calculated from boundary conditions of the fields between the sphere and the surrounding medium. They are:

$$
\begin{gathered}
a_{n}=\frac{\mu m^{2} j_{n}(m x)\left[x j_{n}(x)\right]^{\prime}-\mu_{1} j_{n}(x)\left[m x j_{n}(m x)\right]^{\prime}}{\mu m^{2} j_{n}(m x)\left[x h_{n}^{(1)}(x)\right]^{\prime}-\mu_{1} h_{n}^{(1)}(x)\left[m x j_{n}(m x)\right]^{\prime}} \\
b_{n}=\frac{\mu_{1} j_{n}(m x)\left[x j_{n}(x)\right]^{\prime}-\mu j_{n}(x)\left[m x j_{n}(m x)\right]^{\prime}}{\mu_{1} j_{n}(m x)\left[x h_{n}^{(1)}(x)\right]^{\prime}-\mu h_{n}^{(1)}(x)\left[m x j_{n}(m x)\right]^{\prime}}
\end{gathered}
$$

where $m$ is relative refractive index defined as

$$
m=\frac{n_{1}}{n}
$$

where $n_{1}$ and $n$ are the refractive indices of the sphere and the surrounding medium respectively. $\mu_{1}$ is the permeability of the sphere. $h_{n}^{(1)}$ is the spherical Bessel function of the third kind (also called Hankel function) and is defined as:

$$
h_{n}^{(1)}(\rho)=j_{n}(\rho)+i y_{n}(\rho)
$$

where $j_{n}$ or $y_{n}$ is the spherical Bessel function. Two first orders are:

$$
\begin{gathered}
j_{0}(\rho)=\frac{\sin \rho}{\rho} \quad j_{1}(\rho)=\frac{\sin \rho}{\rho^{2}}-\frac{\cos \rho}{\rho} \\
y_{0}(\rho)=-\frac{\cos \rho}{\rho} \quad y_{1}(\rho)=-\frac{\cos \rho}{\rho^{2}}-\frac{\sin \rho}{\rho}
\end{gathered}
$$

Finally the $x$ is called the size parameter and it is defined as:

$$
x=k a=\frac{2 \pi a}{\lambda}
$$

Each $\mathbf{N}$ and $\mathbf{M}$ correspond to an electromagnetic normal mode of the spherical particle. Therefore the scattered field is a superposition of normal modes, each weighted by the appropriate coefficient $a_{n}$ or $b_{n}$. For each $n$ there are two distinct types of modes: one for which there is no radial magnetic field component, called transverse magnetic modes (TM), and another for which there is no radial electric field component, called transverse electric modes (TE). By looking at equations (4.1) and 
(4.3) one can see that the fields associated to TM modes are weighted by coefficients $a_{n}$ and the fields of TE modes are weighted by coefficients $b_{n}$.

Whenever the size parameter $x$ (and therefore the wavelength) achieves a value for which the denominator of $a_{n}$ or $b_{n}$ becomes very small, the electromagnetic field is dominated basically by one mode and then there exists a resonance. These resonances are enumerated by either TM or TE, and two sub-indexes, $n$ and $m$. Therefore they are written as $\mathrm{TM}_{n, m}$ or $\mathrm{TE}_{n, m}$.

The first sub-index $n$ corresponds to $a_{n}$ or $b_{n}$ coefficients (where $\mathrm{n}=1,2,3, \ldots)$ whose value becomes much higher than the other coefficients. As there can be several values of the size parameter $x$ that maximizes one of the coefficients, the second sub-index $m$ correspond to each of this $x$ values starting from 1 for the lowest $x$ value, 2 for the second one, and so on.

It is interesting to see the distribution of the electric field intensity for different TE and TM modes. Figure 4.4 and figure 4.5 show these distributions in their corresponding resonating planes, as well as in the planes that are perpendicular to the resonating planes. As seen in the figures, the first sub-index of the mode indicates the number of electric field intensity maximums in the half sphere perimeter, and the second one indicates the number of maximums in the radial direction. Figures 4.4 and 4.5 show that in general TE modes are distributed closer to the sphere surface than TM modes. This is an important fact to take into account, for instance, for the placement of an active material that is expected to interact with the cavity mode.

The scattering cross section is a very important parameter when comparing theory to experiment. It can be calculated from energy conservation rules and using the above development of the fields. A very useful way of writing this parameter is as follows:

$$
\sigma_{s c a}=\frac{2 \pi}{k^{2}} \sum_{n=1}^{\infty}(2 n+1)\left(\left|a_{n}\right|^{2}+\left|b_{n}\right|^{2}\right)
$$




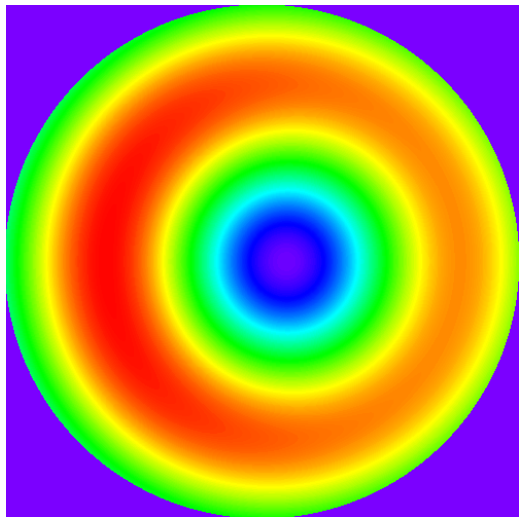

(a)

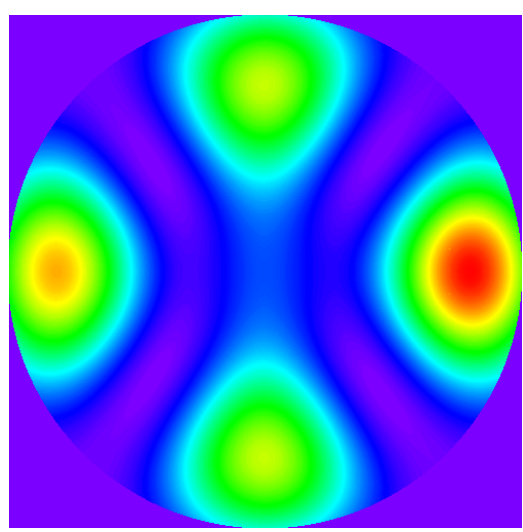

(c)

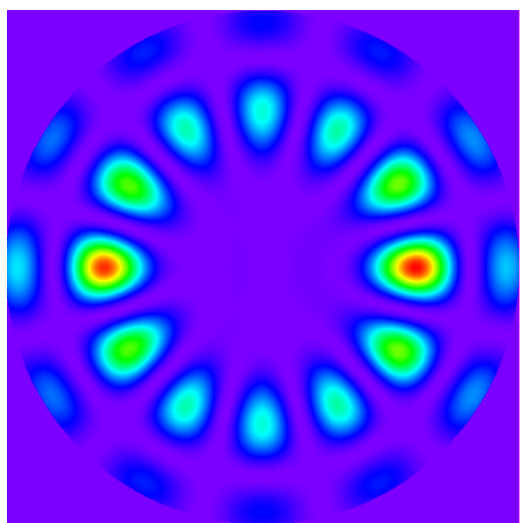

(e)

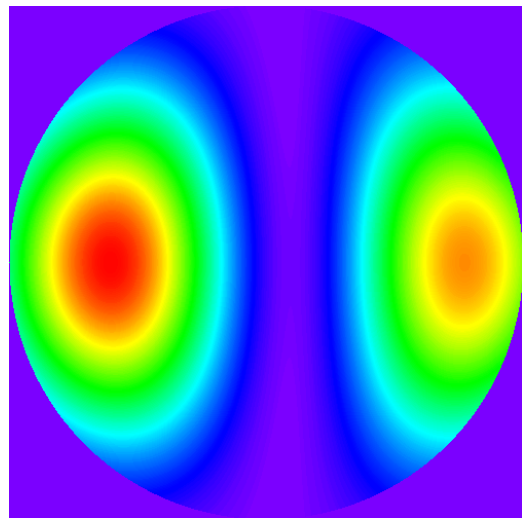

(b)

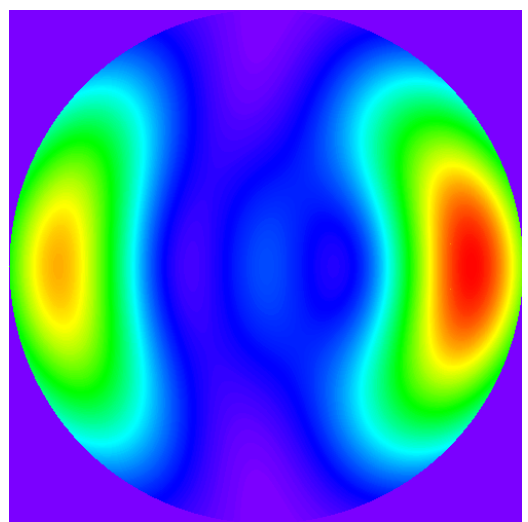

(d)

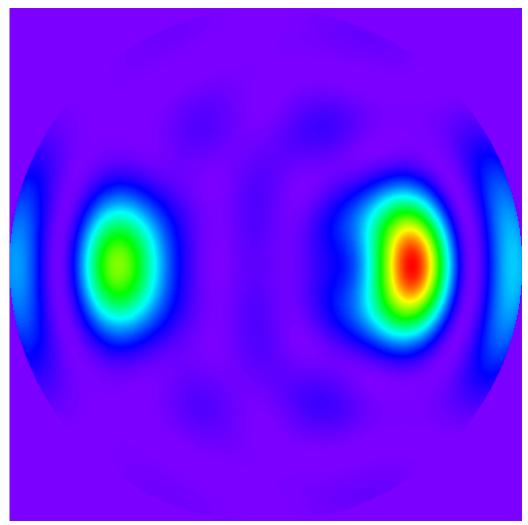

(f)

Figure 4.4: Electric field intensity distribution of TE resonant modes. The left images correspond to the resonant planes and the right images correspond to the planes perpendicular to the resonating planes. (a) and (b) $\mathrm{TE}_{1,1}$ mode, (c) and (d) $\mathrm{TE}_{2,1}$ mode and (e) and (f) $\mathrm{TE}_{6,2}$ mode. Zero intensity is indicated by blue color and the highest intensity is indicated by red color. 


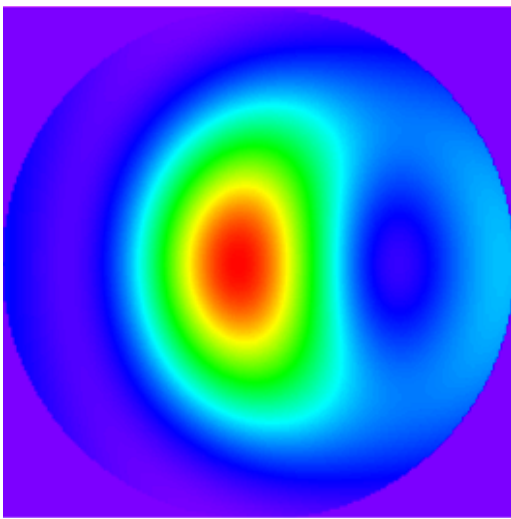

(a)

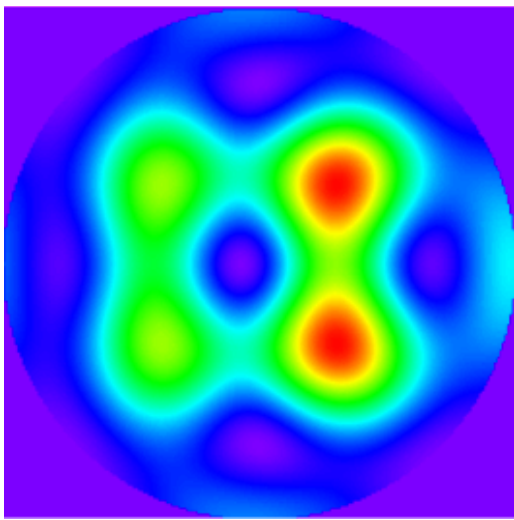

(c)

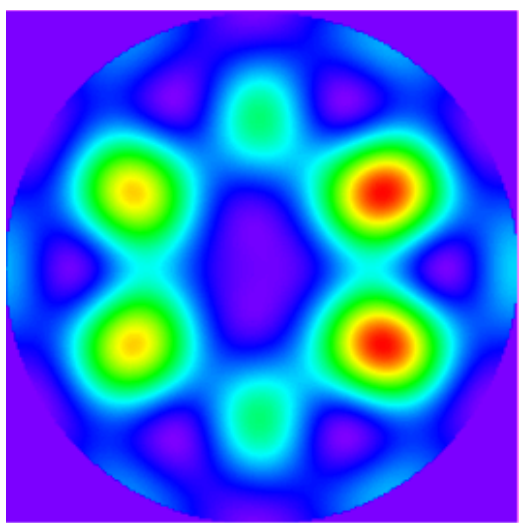

(e)

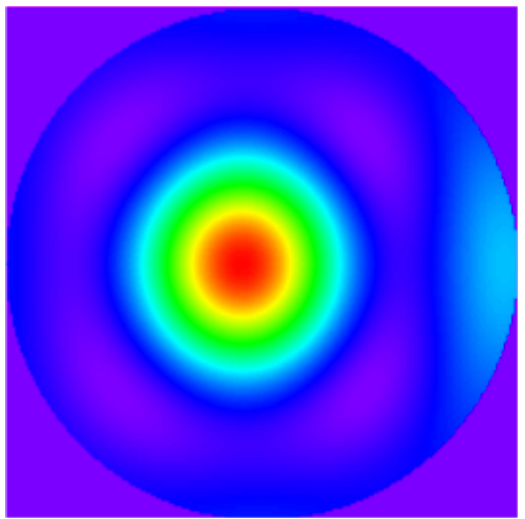

(b)

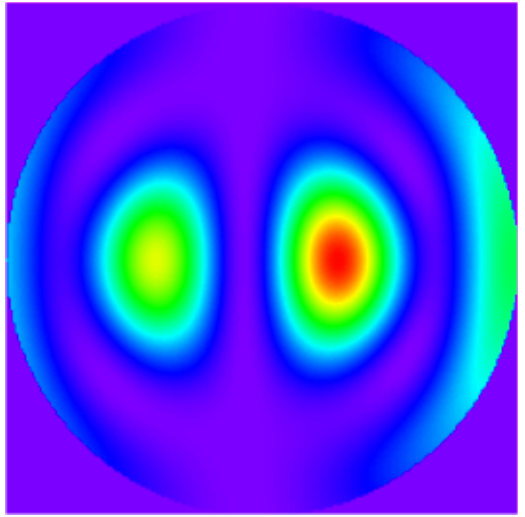

(d)

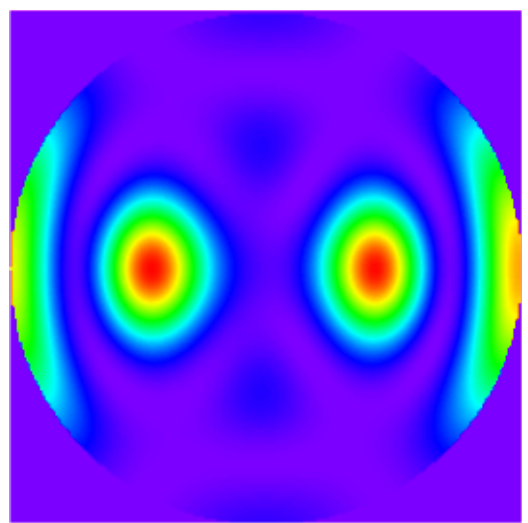

(f)

Figure 4.5: Electric field intensity distribution of TM resonant modes. The left images correspond to the resonant planes and the right images correspond to the planes perpendicular to the resonating planes. (a) and (b) $T M_{1,1}$ mode, (c) and (d) $\mathrm{TM}_{2,1}$ mode and (e) and (f) $\mathrm{TM}_{3,1}$ mode. Zero intensity is indicated by blue color and the highest intensity is indicated by red color. 
Nowadays there are subrutines available for performing calculations of the scattering cross section, etc. that have been used in this thesis. Particularly we have used the one of P.W. Barber and S.C. Hill [8] for calculating scattering efficiency $Q_{\text {sca }}$ which is related to scattering cross section $\sigma_{s c a}$ by the following relation:

$$
Q_{s c a}=\frac{\sigma_{s c a}}{\pi a^{2}}
$$

where $a$ is the radius of the sphere. $Q_{\text {sca }}$ tells us how large is the area of the particle as far as optical scattering is concerned, in comparison with the geometric area projected by the particle $\left(\pi a^{2}\right)$. We will see below that this optical area can be several times bigger than the geometric area.

The optical transmittance spectrum of a silicon colloid is determined by its scattering cross section $\sigma_{\text {sca }}$ as we will see in section 4.3.1.2. Figure 4.6 shows the calculation of the scattering cross section $\sigma_{\text {sca }}$ of a $2 \mu \mathrm{m}$ silicon sphere, as well as its scattering efficiency $Q_{s c a}$. Both are plotted against wavelength (up axis) and size parameter (bottom axis). Each peak corresponds to a resonating mode which is indicated by using the nomenclature explained above.

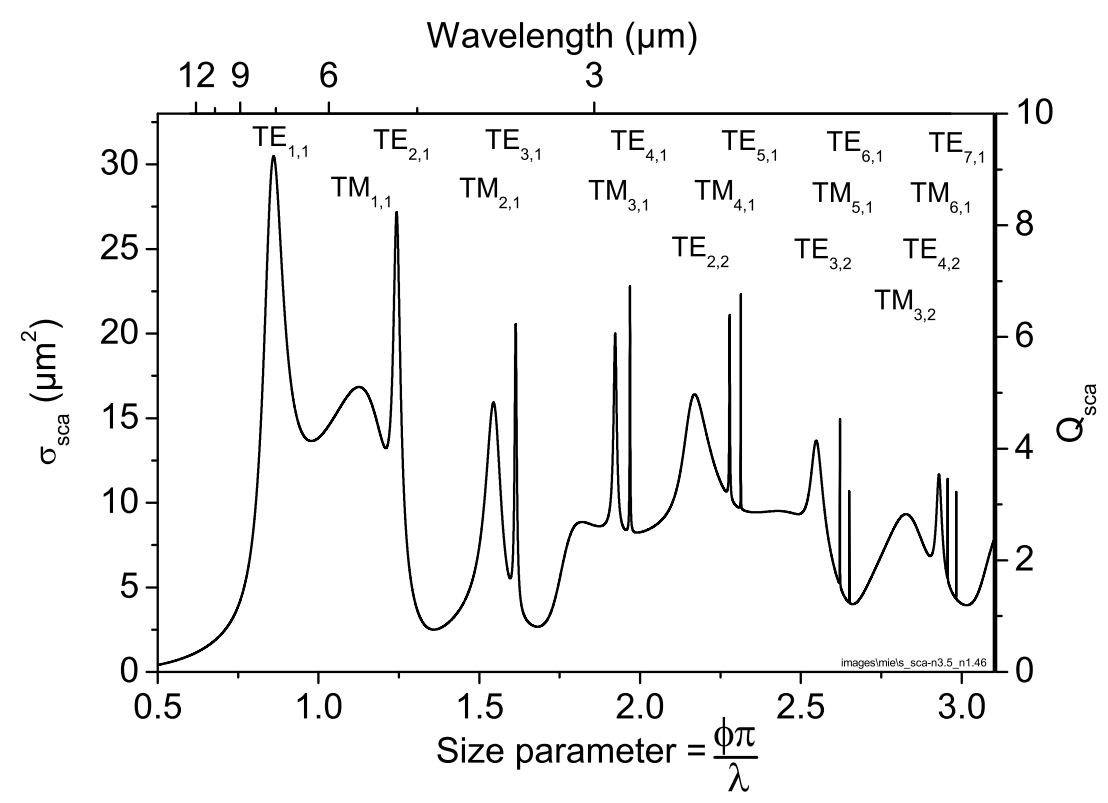

Figure 4.6: Calculated $\sigma_{s c a}$ and $Q_{s c a}$ for a sphere of $2 \mu \mathrm{m}$ in diameter made of silicon $(n=3.5)$. The resonant modes are indicated on their corresponding peaks. 


\subsubsection{The influence of the refractive index on the resonance phenomena}

As we mentioned in section 4.2.2, the underlying mechanism of the light trapping effect in a spherical cavity is the total internal reflection of light. This physical phenomenon depends strongly on the refractive index contrast between the sphere and the surrounding medium. While a low contrast produces losses of light, a high contrast makes this mechanism more effective, i.e. the losses of light in the wall of the sphere are much smaller. In order to better understand how this influences the resonating modes of a cavity, figure 4.7 shows a calculation by Mie theory of the scattering cross section for two spheres having a diameter of two micrometers. One of them is made of silicon (refractive index $n=3.50$, black line) and the other one is made of silica (blue line) $(n=1.46)$. While the silicon sphere shows clear resonances for low size parameters associated to low $m, n$ values (see the nomenclature of the resonances above), they can hardly be appreciated in the scattering cross section produced by the silica microsphere. Also, the resonances associated to the silicon microsphere are much sharper than those of the silica microsphere.

For instance when comparing the resonating mode $\mathrm{TE}_{8,2}$ for a silicon microsphere and mode $\mathrm{TE}_{4,2}$ for a microsphere made of silica (size parameter 4.38 and 4.56 respectively, see figure 4.8) the $Q$ value for the silicon microsphere is approximately 22000 while for the silica microsphere the $Q$ value is approximately 300 . This means that for the same size, the silicon particle will confine much more energy then the silica microsphere. 


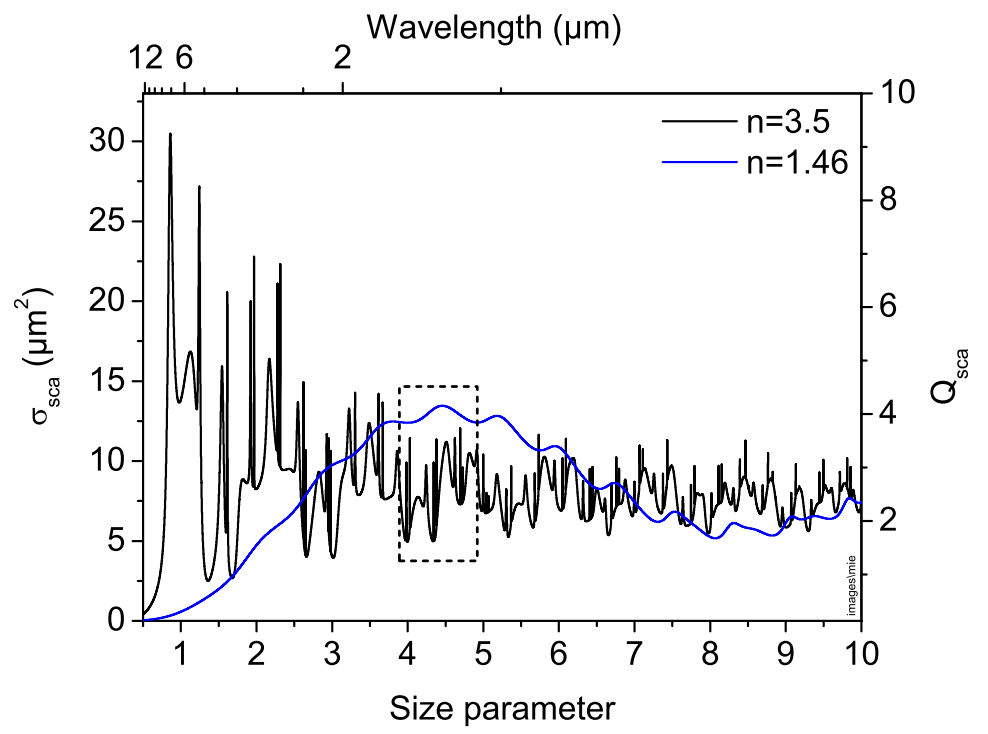

Figure 4.7: Comparing calculated scattering cross section $\sigma_{\text {sca }}$ for a sphere of $2 \mu \mathrm{m}$ in diameter made of silica $\mathrm{n}=1.46$ (blue) and silicon $\mathrm{n}=3.5$ (black). The dashed region is shown amplified in figure 4.8.

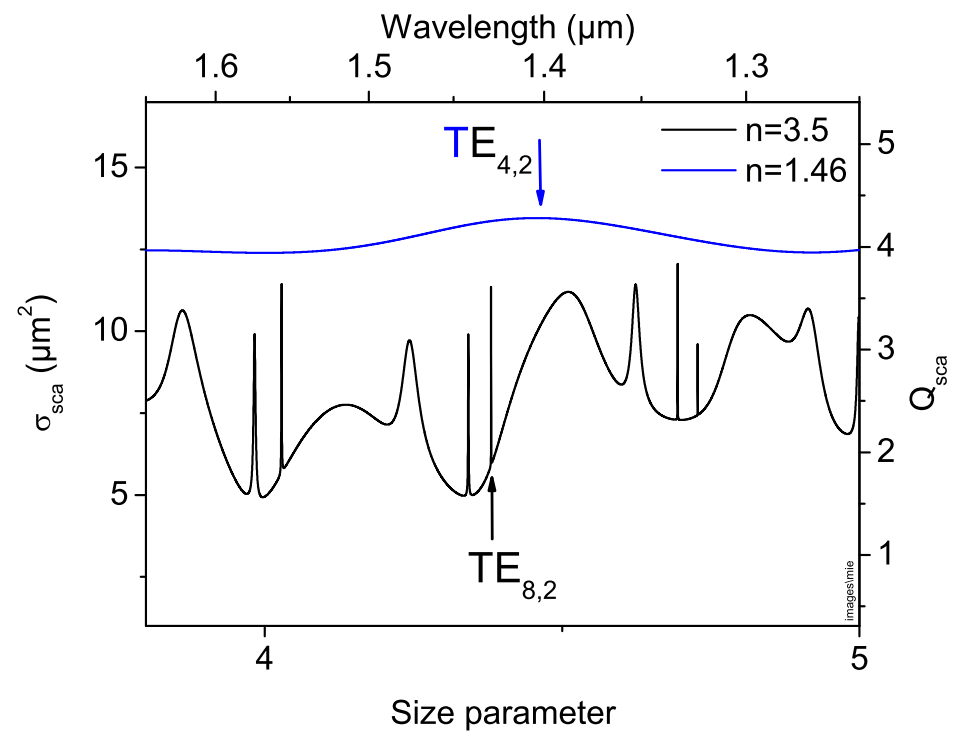

Figure 4.8: Scattering cross section $\sigma_{s c a}$ for a sphere of $2 \mu \mathrm{m}$ in diameter made of silica $\mathrm{n}=1.46$ (blue) and silicon $\mathrm{n}=3.5$ (black). The $\mathrm{TE}_{8,2}$ mode (indicated with an arrow) for the silicon microsphere has a $Q$ value that is approximately 22000 and the $\mathrm{TE}_{4,2}$ mode for a microsphere made of silica has a $Q$ value of approximately 300 . 


\subsection{Experimental results}

\subsubsection{Optical properties of single silicon colloids}

\subsubsection{Experimental setup}

The method of optical transmittance was chosen for characterizing the resonant modes of silicon colloids. For this purpose a Bruker IFS 66/S fourier transform spectrometer with a coupled microscope was used. This spectrometer allows measuring optical transmittance in the wavelength range from 0.5 to $4 \mu \mathrm{m}$. Figure 4.9 shows the scheme of the experimental setup. Many silicon colloids are put onto a microscope slide substrate, which can be visualized by the microscope. This substrate is transparent in all the wavelength range where the measurements take place and its low refractive index (1.45) should barely influence the optical properties of the silicon microspheres. We have used a Cassegrain 15X objective that projects the image of the colloids on a diaphragm whose minimum available aperture is $20 \mu \mathrm{m}$. This reduced aperture makes the detected light intensity be very low, but the size of the diaphragm is still much larger than the sphere diameter values, which are typically $0.5-3.5 \mu \mathrm{m}$. Because of these reasons, the obtained optical transmittance spectra are very noisy and with a very small dynamic range or contrast as we will see below. Moving the microscope platform where the substrate is supported allows choosing an area that contains a single silicon colloid. Then, the transmitted light through this area is measured. This signal is divided by the background that is recorded by measuring the transmitted signal through an adjacent area of the substrate that does not containing any silicon colloid. In order to reduce the noise of the signal and reasonably solve the features of the spectra we utilized long integration times, from 15 to 30 minutes. In all cases we used non polarized light. 


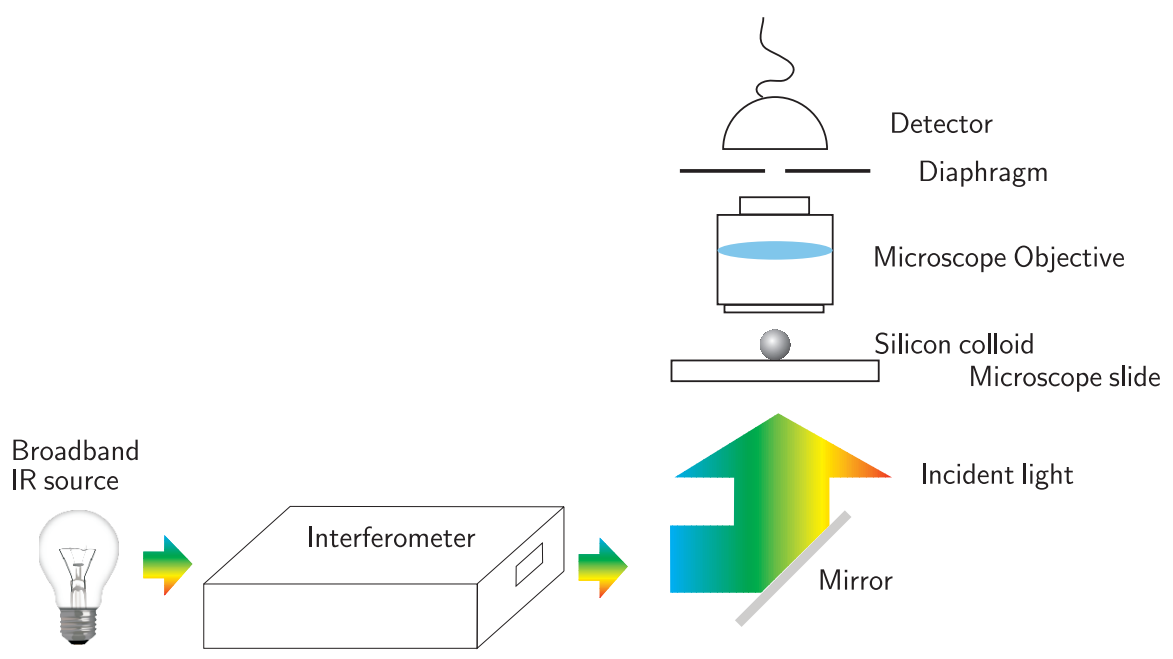

Figure 4.9: Simplified schematic of the Bruker Infrared Fourier Transform Spectrometer used for measuring the optical transmittance of single silicon colloids. The spectrometer consists of an infrared unpolarized broadband light source, an interferometer and a microscope. The light from the IR source is coupled to the interferometer. From the interferometer the light is passed via mirrors and through the substrate which contains silicon colloids. Further, the scattered light is projected onto the diaphragm and finally, detected by an infrared Mercury Cadmium Telluride (MCT) detector.

\subsubsection{The obtained spectra and their fit to Mie theory}

Optical transmittance of silicon colloids of different types: amorphous and polycrystalline ones, and having different diameters were measured (see black curves in figures 4.12 to 4.15 ). The spectra were fit to Mie theory by using the following equation:

$$
T=1-\frac{\sigma_{\text {sca }}}{A_{\text {pin }}}
$$

where $T$ is transmittance, $\sigma_{s c a}$ is the scattering cross section and $A_{p i n}$ is the area of the pinhole.

Figure 4.10 shows a scheme of the different areas taking part in equation (4.12). $A_{\text {pin }}$ is much larger than $\sigma_{s c a}$, and $\sigma_{\text {sca }}$ can be larger than the physical area projected by the sphere. 


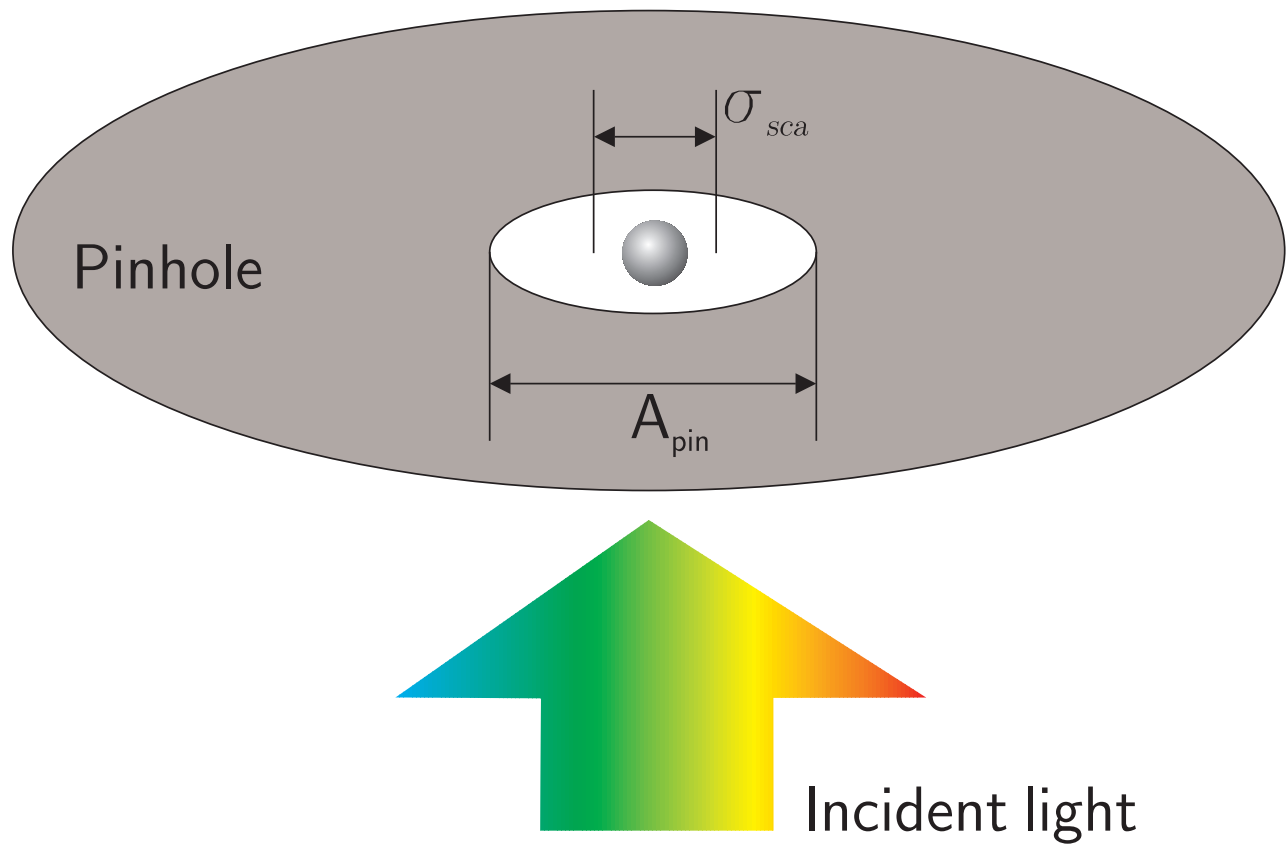

Figure 4.10: Relation between pinhole diameter $A_{\text {pin }}$ and scattering cross section $\sigma_{s c a} . \sigma_{s c a}$ can be larger than the physical area projected by the sphere.

The values of $\sigma_{\text {sca }}$ were calculated by using equation (4.10) and the refractive index values of silicon. Therefore, the only fitting parameter is the sphere diameter. The actual fitting process was done in MS Excel because it provides an interface where the variation produced in a curve by a fitting parameter can be readily visualized. A subrutine created by P.W Barber and S.C. Hill [8] is called from MS Excell for the calculation of $\sigma_{\text {sca }}$.

In case of polycrystalline silicon the refractive index values given by Palik for crystalline silicon, were used. Figure 4.11 shows the dispersive relation. Because only several discrete values are given, we interpolated the points in between whenever it was necessary. For spheres made of amorphous silicon, the refractive index values given by Palik did not fit so well. Instead, the best fitting was achieved by using a refractive index value of 3.24 in all the measured range. This is not surprising because the optical properties of amorphous silicon depend strongly on the particular method and parameters used to synthesize it. In both cases, poly-crystalline and amorphous, we assumed no imaginary part of the refractive index; i.e.: we assumed zero optical absorption. As we will 
see later, this assumption is not always supported by the experimental results.

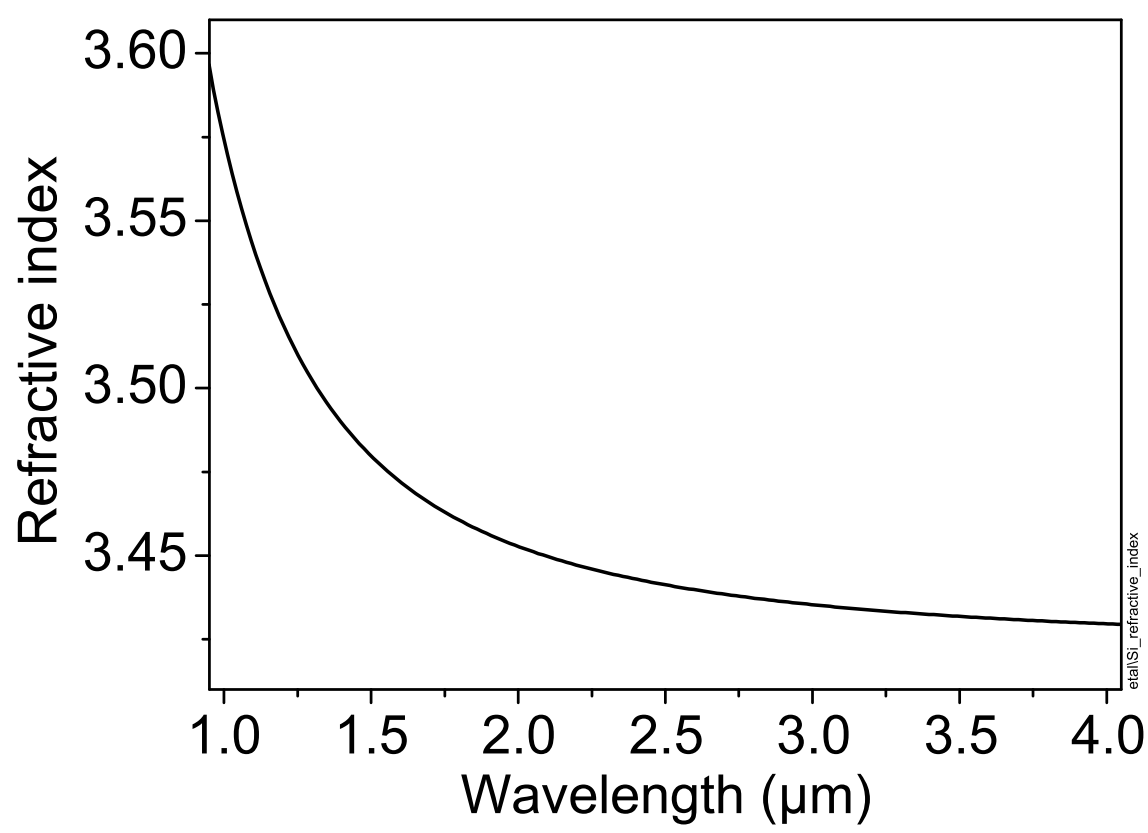

Figure 4.11: Refractive index for crystalline silicon versus wavelength according to values given by Palik [9].

The results of the experimental optical transmittance and the fitted curves are presented in figures 4.12 to 4.15. Experimental data are shown in black and the calculated fit in orange colour. Each deep corresponds to an optical Mie resonance, or Whispering Gallery Mode. Actually, a deep in transmittance corresponds to a peak in the $\sigma_{\text {sca }}$ curve (see figure 4.6). Similarly to figure 4.6, the resonances are now enumerated under their corresponding deep in figure 4.12. Although the measurements were performed in the range from 1 to 4 micrometers, the calculation is extended further in the infrared to show where the modes start appearing, i. e. to show the position of the first mode $\left(\mathrm{TE}_{1,1}\right)$. The fitted values of sphere diameter have been summarized in table 4.1. 


\begin{tabular}{ccc}
\hline Sphere diameter $(\mu m)$ & Silicon type & Figure number/On page \\
\hline 1.050 & Polycrystalline & $4.12 / 75$ \\
1.885 & Polycrystalline & $4.13 / 76$ \\
3.350 & Polycrystalline & $4.14 / 77$ \\
4.000 & Amorphous & $4.15 / 78$ \\
\hline
\end{tabular}

Table 4.1: Summary of fitted sphere diameter values. The type of silicon the spheres are made of, and the corresponding figure number and page where the figure appears are indicated in columns 2 and 3 respectively.

The modes position in size parameter is the same no matter the diameter of the sphere, except for some small variation due to the refractive index dispersion (see figure 4.11), however the diameter of the sphere determines the position of the modes in the wavelength space. For instance, the $\mathrm{TE}_{1,1}$ occurs at about a size parameter of 0.8 . This means a position in wavelength of around 4 micrometer for a sphere of 1.050 micrometers in diameter (figure 4.12) and a position of about 6.5 micrometers for a sphere of 1.885 micrometers (figure 4.13). 


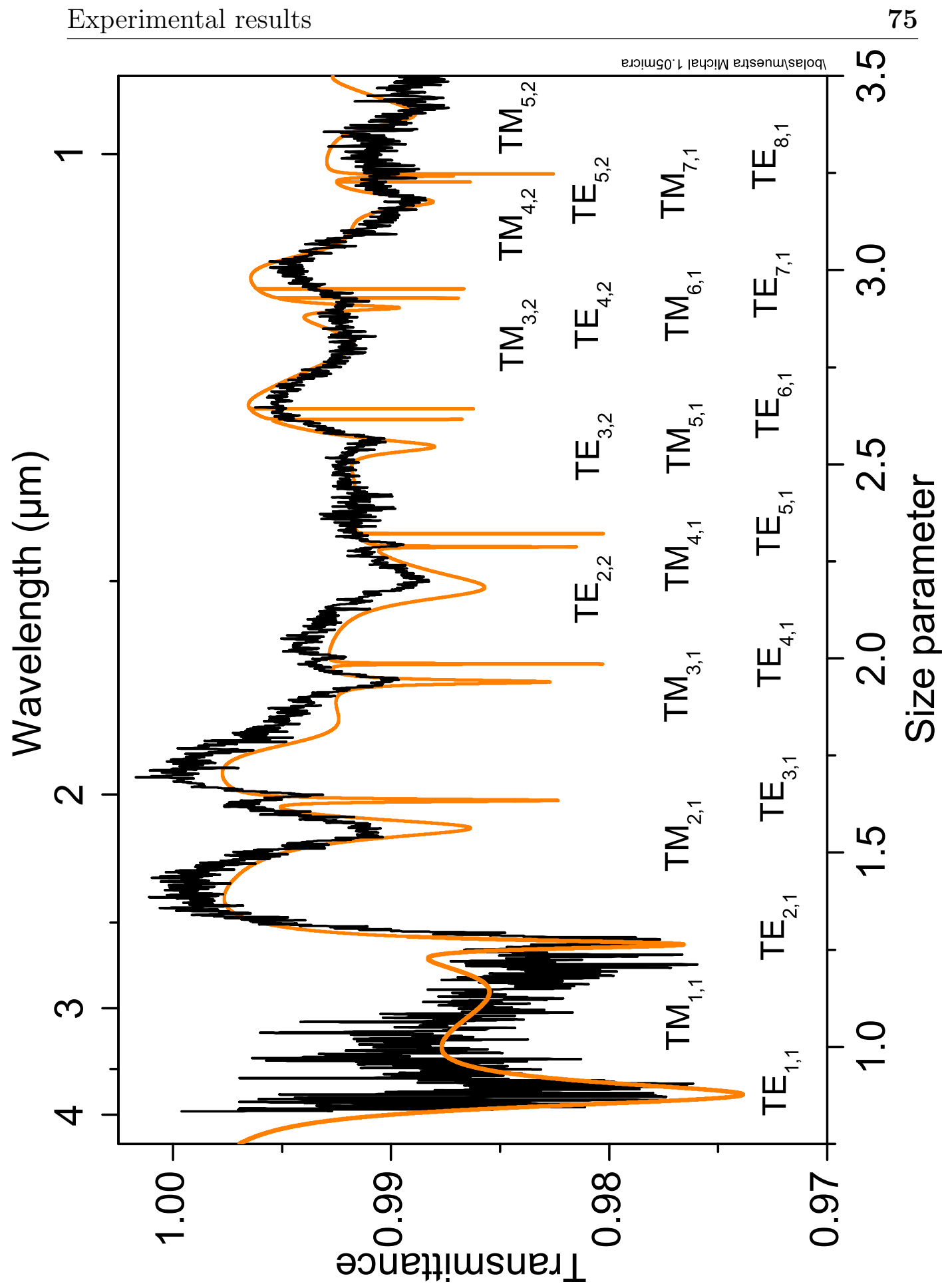

Figure 4.12: Experimental optical transmittance (black curve) and calculated fit (orange colour) for a polycrystalline single silicon colloid grown at $400{ }^{\circ} \mathrm{C}$ during 10 minutes and recrystallized at $800{ }^{\circ} \mathrm{C}$ during 1 hour. The resulting fitted diameter is $\phi=1.050 \mu \mathrm{m}$. Each deep corresponds to a Mie resonance in a similar way as the peaks of $\sigma_{s c a}$ in figure 4.6. The resonances are indicated here again by using the same nomenclature. 
Optical properties of silicon colloids

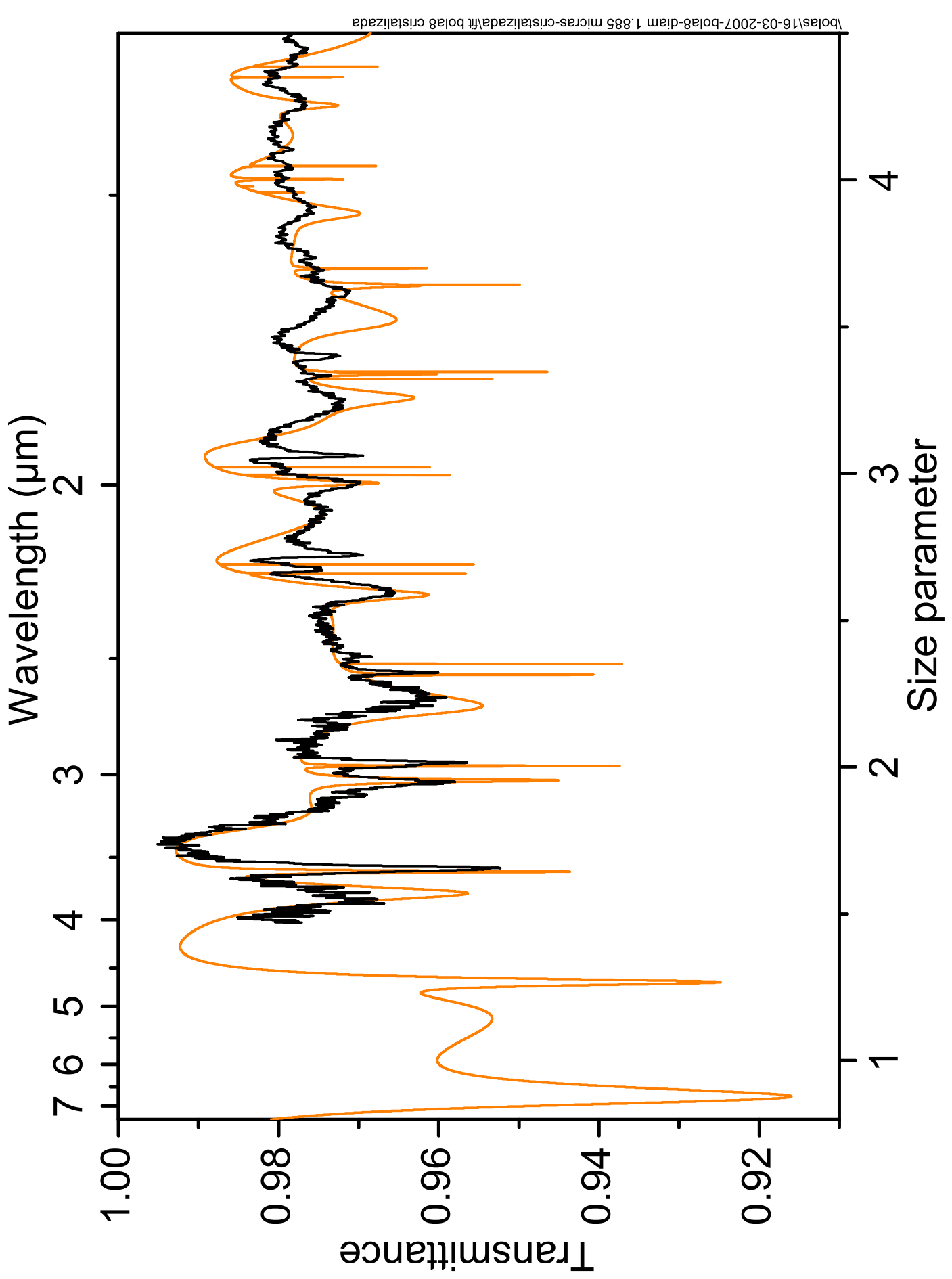

Figure 4.13: Experimental optical transmittance (black curve) and calculated fit (orange colour) for a polycrystalline single silicon colloid grown at $400{ }^{\circ} \mathrm{C}$ during 1 hour and recrystallized at $800{ }^{\circ} \mathrm{C}$ during 1 hour. The resulting fitted diameter is $\phi=1.885 \mu \mathrm{m}$. 


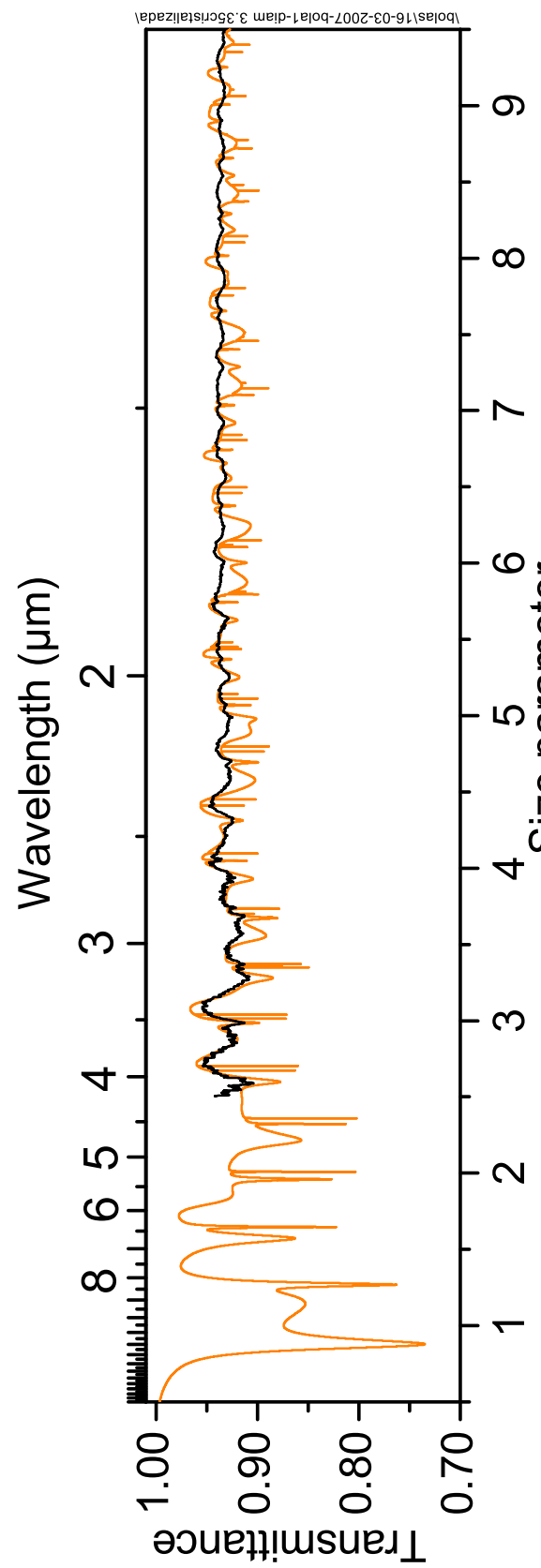

(a)

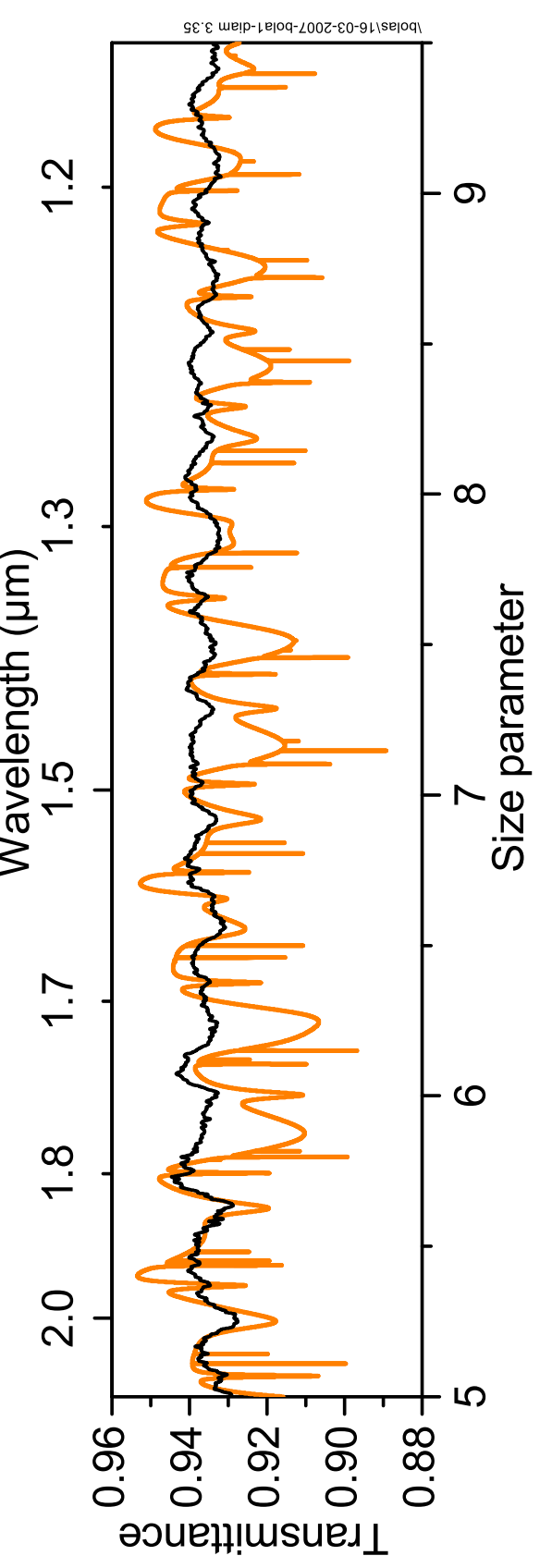

(b)

Figure 4.14: (a) and (b) experimental optical transmittance (black curve) and calculated fit (orange colour) for a polycrystalline single silicon colloid grown at $400{ }^{\circ} \mathrm{C}$ during 30 minutes and recrystallized at $800{ }^{\circ} \mathrm{C}$ during 1 hour. The resulting fitted diameter is $\phi=3.350 \mu \mathrm{m}$. 


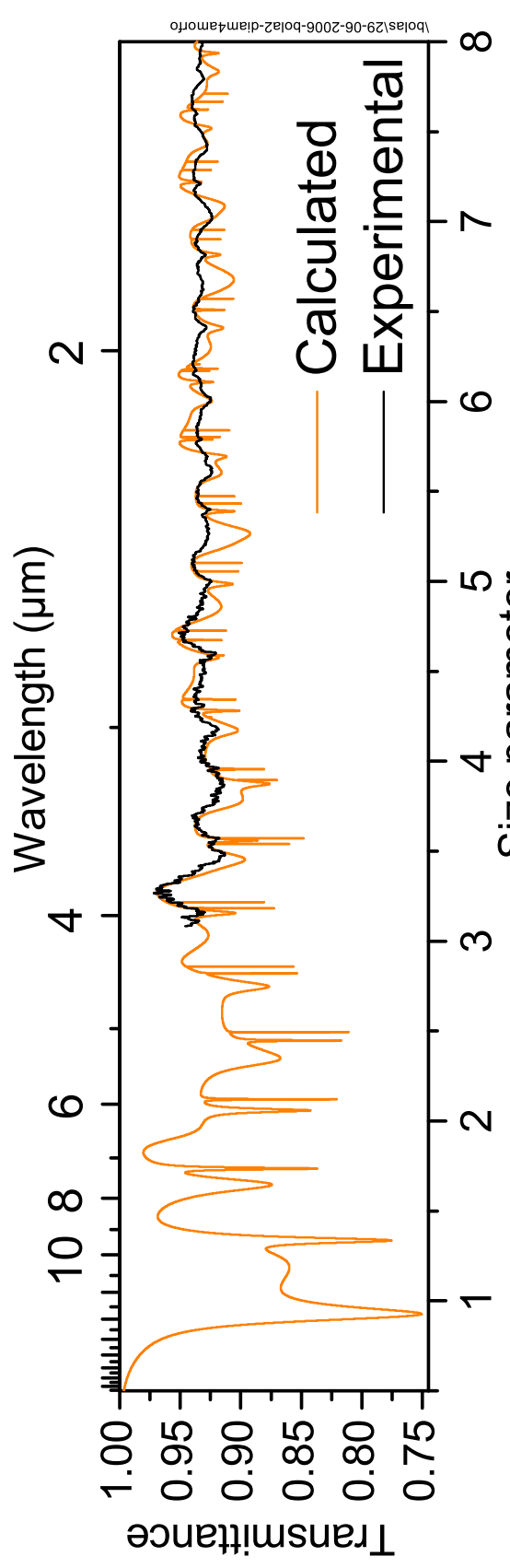

(a)

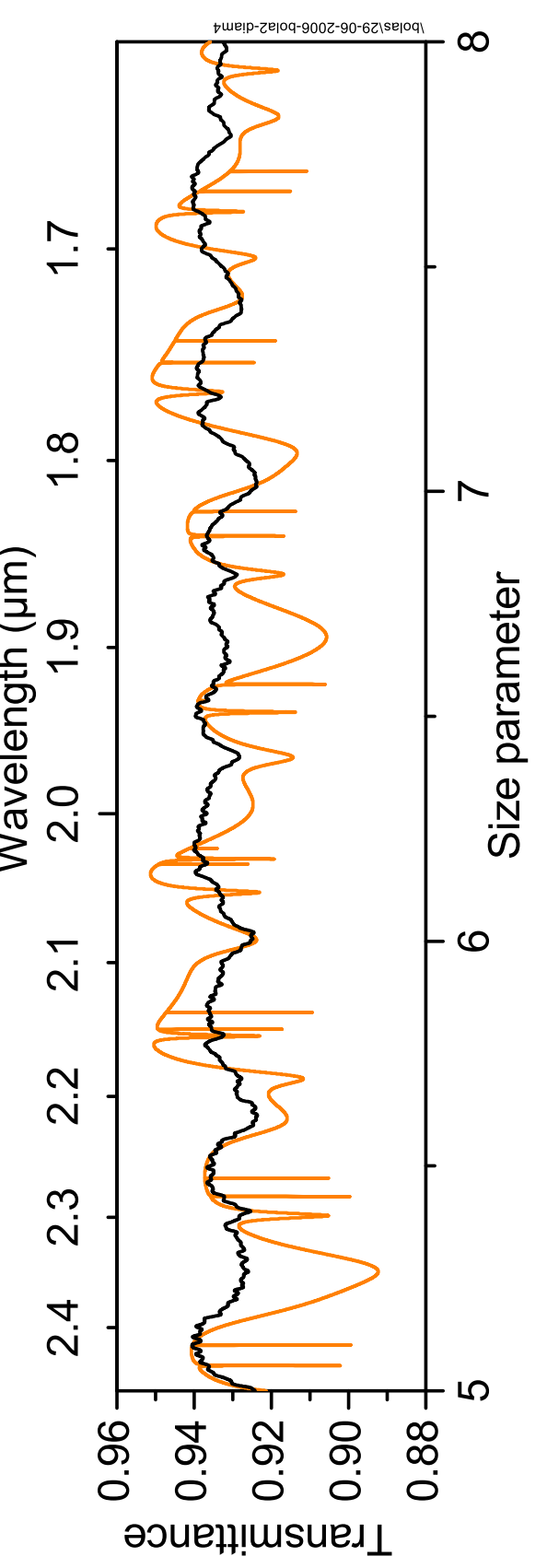

(b)

Figure 4.15: (a) and (b) experimental optical transmittance (black curve) and calculated fit (orange colour) for an amorphous single silicon colloid, grown at $450{ }^{\circ} \mathrm{C}$ during 1 hour. The resulting fitted diameter is $\phi=4.050 \mu \mathrm{m}$. 
As figures $4.12,4.13,4.14$ and 4.15 show, there is a clear agreement between theory and experiment across the whole wavelength range of measurement. These results confirm that microspheres are actually made from silicon and demonstrate that they are in good condition (highly spherical and absent surface roughness). This allowed fitting of the diameter of the spheres precisely, up to the nanometer. However, a closer look at any of the figures 4.12, 4.13, 4.14 and 4.15 reveals small differences between experiment and theory. Mie peaks at shorter wavelength (large size parameter) fit worse than those at longer wavelength (small size parameter) values. This can be due to the influence of optical absorption in the samples. If impurities are present in the sample optical absorption in the gap would take place, and due to thermal effects, it would produce larger effects near the fundamental absorption region of silicon (i.e.: at larger size parameter values).

The electromagnetic energy that is localized in a microsphere at a resonant frequency (see figures 4.4 and 4.5 ) values is directly proportional to the quality factor $Q$ of the resonant mode. The $Q$ of a mode can be calculated as $\frac{\lambda_{0}}{\Delta \lambda}$, where $\lambda_{0}$ and $\Delta \lambda$ are position and width (FWHM), respectively, of the associated deep.

We have compared the $Q$ factor for modes $\mathrm{TM}_{2,1}, \mathrm{TE}_{3,1}$ and $\mathrm{TM}_{4,1}$ for those spheres with diameter $1.050 \mu \mathrm{m}$ and $1.885 \mu \mathrm{m}$. The theoretical and experimental $Q$ factors are presented in table 4.2 below:

\begin{tabular}{lcccc}
\hline & \multicolumn{2}{c}{ Theoretical $Q$ factor } & \multicolumn{2}{c}{ Experimental $Q$ factor } \\
\cline { 2 - 5 } Mode & \multicolumn{2}{c}{ Sphere diameter } & \multicolumn{2}{c}{ Sphere diameter } \\
& $\phi=1.050 \mu \mathrm{m}$ & $\phi=1.885 \mu \mathrm{m}$ & $\phi=1.050 \mu \mathrm{m}$ & $\phi=1.885 \mu \mathrm{m}$ \\
\hline $\mathrm{TM}_{2,1}$ & 25 & 25 & $14(2.101)$ & $15(3.772)$ \\
$\mathrm{TE}_{3,1}$ & 195 & 186 & $41(2.018)$ & $56(3.604)$ \\
$\mathrm{TM}_{4,1}$ & 953 & 818 & $63(1.442)$ & $298(2.558)$ \\
\hline
\end{tabular}

Table 4.2: Theoretical and experimental $Q$ factors for two silicon spheres with diameter of $\phi=1.050 \mu \mathrm{m}$ and $\phi=1.885 \mu \mathrm{m}$. The numbers in parenthesis indicate the wavelength in micrometers where the resonance deeps are centered.

As seen in table 4.2, the experimental $Q$ values are always below the theoretical ones. Achieving a complete explanation of this fact is beyond the scope of this thesis. Instead, we give a qualitative explana- 
tion: Firstly, the observed decrease of $Q$ for the three modes can not be attributed to the limited resolution of the spectrometer, because this resolution is about $1 \mathrm{~nm}$ and it corresponds to a $Q$ value of around 1000, which is higher than the theoretical $Q$ value of the modes. Secondly, we think the most important effect that contributes to the decrease of $Q$ is the optical absorption of silicon, which is provoked by the existence defects in the electronic gap, presumably due to defects in the crystalline arrangement of atoms, contaminations, etc. This could explain why the difference between theoretical values and experimental ones increases as the wavelength at which the resonance occurs becomes shorter. For instance, the experimental $Q$ value of mode $\mathrm{TM}_{4,1}$ is much closer to the theoretical value for the $1.885 \mu \mathrm{m}$ diameter sphere (mode position at $\lambda=2.558 \mu \mathrm{m}$ ) than for the $1.050 \mu \mathrm{m}$ sphere (mode position at $\lambda=1.442 \mu \mathrm{m})$. Thirdly, other factors that may contribute to the decrease of $Q$ values are surface defects like residual roughness, etc. This type of defects should influence more strongly those modes where the electric field is localized closer to the sphere surface (for instance mode $\mathrm{TM}_{4,1}$ ) and it should influence much less those modes where the electromagnetic field is localized more inside the sphere (for instance modes $\mathrm{TM}_{2,1}$ and $\mathrm{TE}_{3,1}$ ). In any case, researching the cause of the difference between experimental and theoretical $Q$ factors is a very important issue and it constitutes an object of future research. Although at this initial stage high $Q$ factors could not be achieved, the micro-spheres can yield theoretically very high values, for instance, the theoretical $Q$ factor for mode $\mathrm{TE}_{8,1}$ is about $6 \cdot 10^{5}$, and other modes at higher size parameters can reach even higher $Q$ factors. 


\subsubsection{Optical properties of photonic sponges}

We have seen in chapter 2 that during the synthesis process of silicon colloids, they aggregate by gravity onto a substrate forming a photonic sponge. This is an open structure with silicon microspheres placed one on another, forming treelike arrangements as shown in figure 4.16.

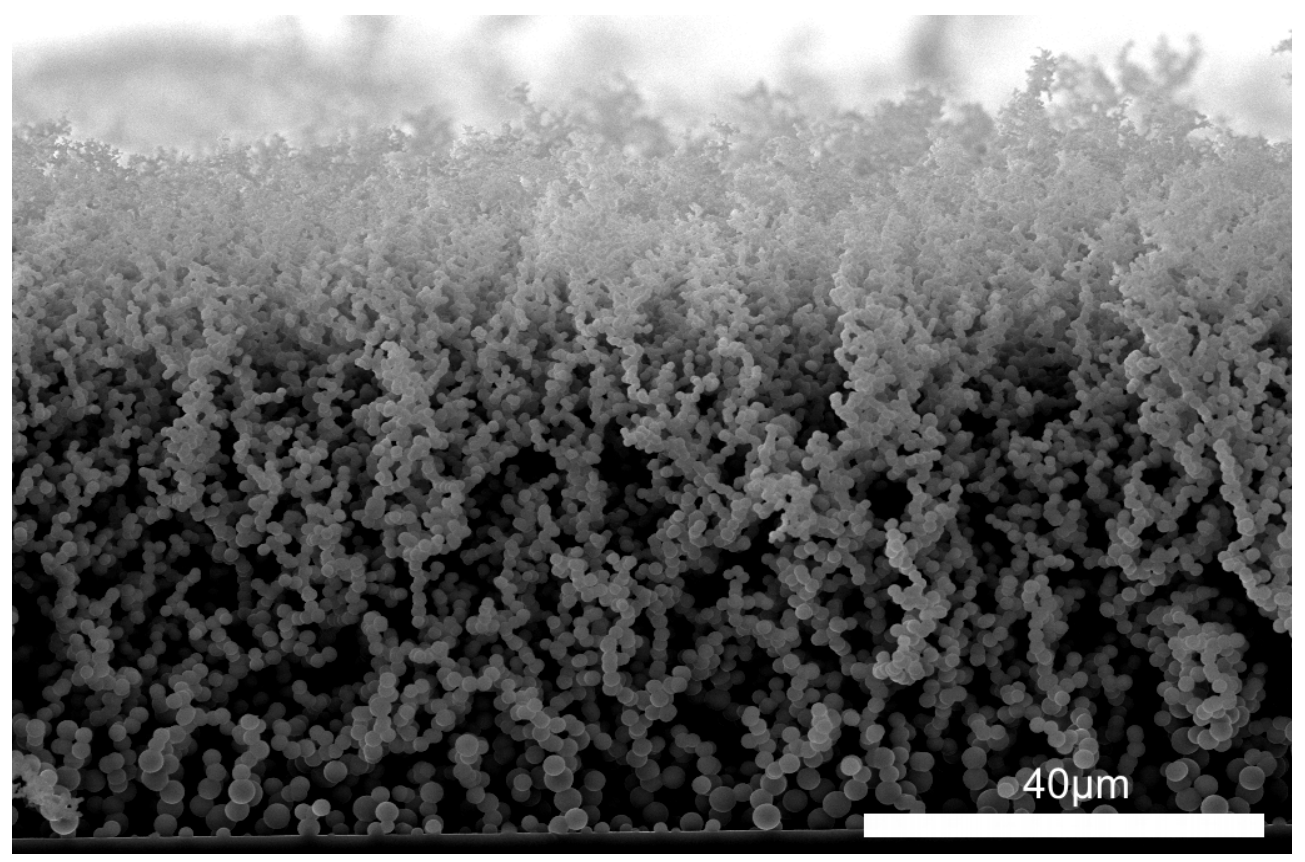

Figure 4.16: A cross section view of a photonic sponge deposited on a silicon wafer.

Of course, a photonic sponge does not have to be fabricated as mentioned above but it can also be obtained by other means, i.e. the concept of photonic sponge is not limited to the type of structures obtained by the CVD process of chapter 2 (although this is our main goal) but it can be extended to structures with different filling fractions, or where the spheres are not touching each other, or the spheres are dispersed in a liquid solution, etc. Here, we will study the influence of some structural parameters on the optical properties of photonic sponges based on silicon colloids. These structural parameters are:

1. Sphere diameter distribution

2. Filling fraction (distance between the spheres)

3. Thickness of the photonic sponge layer 
In the case of the first parameter, the "usual" sponges, obtained during the CVD process of synthesis of silicon colloids, were used. We call them "as grown photonic sponges" from now on. In the last two cases, because the filling fraction and thickness could not be varied in a controlled way, a new type of sponge was used. It consist of solid solutions of silicon colloids, where the embedding medium is Potassium Bromide. In both cases we have performed optical transmittance measurements by using a Nicolet 710 Fourier Transform Infra Red spectrometer (FTIR), that is able to measure in a wide spectral range from 2.5 to 35 micrometers of wavelength. The light source in the FTIR is a glow bar which emits totally depolarized light. The Infra Red (IR) beam impinges perpendicular to the sample on a surface area of about $1 \mathrm{~cm}$ in diameter, and the scattered light is collected in a narrow cone in the normal direction to the sample surface. 


\subsubsection{The influence of the sphere size distribution}

The study of the influence of the sphere size distribution was performed by choosing as grown photonic sponges having nearly the same thickness and the same filling fraction, but different sphere size distribution. Table 4.3 summarizes the structural parameters of two chosen sponges. Such parameters were measured by SEM after having performed the optical transmittance measurements. The sponges were deposited on silicon slabs of 550 micrometers in thickness and having the two sides polished. The silicon slab is transparent throughout the spectral region from 2.5 to $35 \mu \mathrm{m}$ except in the range around $16 \mu \mathrm{m}$, where $\mathrm{Si}-\mathrm{Si}$ and $\mathrm{Si}-\mathrm{H}$ vibrations are IR-active [10] as shown in figure 4.17. Transmittance of $55 \%$ is due to reflection at the silicon surface.

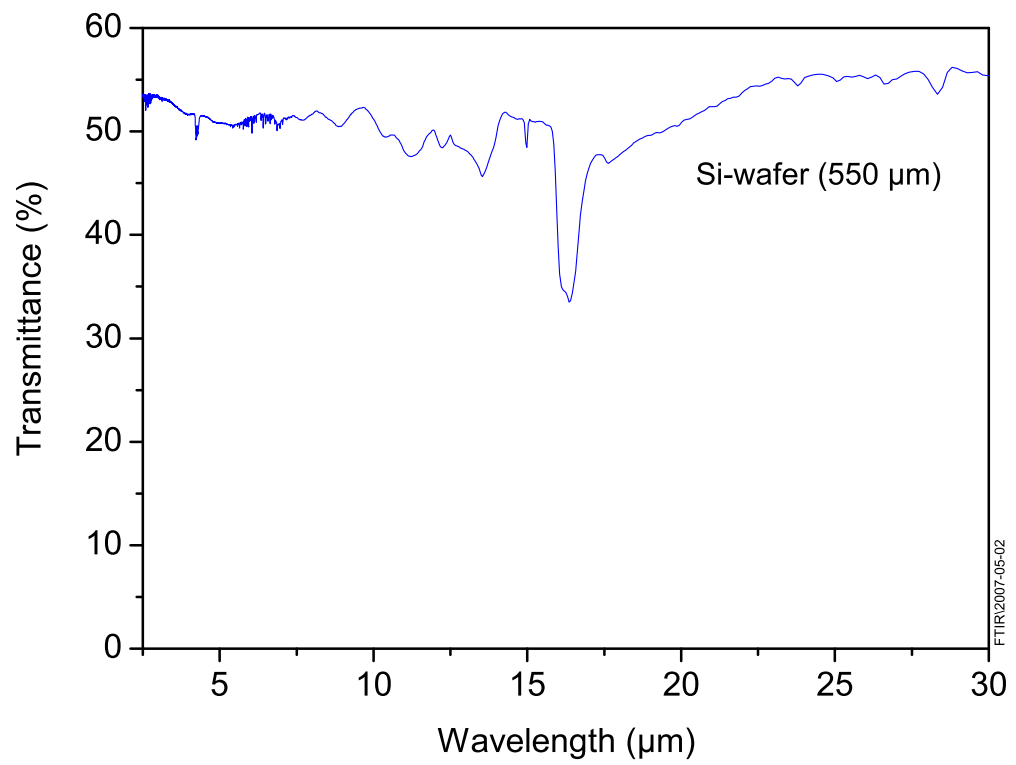

Figure 4.17: Optical transmittance of the silicon slab where sponges where deposited by CVD means. The slab is polished on both sides and it is $550 \mu \mathrm{m}$ thick. The large absorbance deep around $16 \mu \mathrm{m}$ is where $\mathrm{Si}-\mathrm{Si}$ and $\mathrm{Si}-\mathrm{H}$ phonons are IR-active.

The histograms illustrating the different sphere size distribution of the two sponges are shown in figure 4.18(a) (Sponge A) and figure 4.18(b) (Sponge B), and the measured optical transmittance curves are shown in figure 4.19 in continuous lines for sponge A (green) and sponge B (red). While the silicon slab produces a flat curve in the transmittance spectrum, except the deeps associated to phonons (see figure 4.17) the 


\begin{tabular}{ccccc}
\hline Sponge & $\begin{array}{c}\text { Deposition } \\
\text { time (hours) }\end{array}$ & Thickness $(\mu m)$ & Mode $(\mu m)$ & $\begin{array}{c}\text { Filling } \\
\text { fraction }(\%)\end{array}$ \\
\hline A & 0.17 & 9 & 1.3 & 5 \\
B & 1 & 13 & 1.3 & 5 \\
\hline
\end{tabular}

Table 4.3: Structural properties of photonic sponges used to study the influence of the sphere size distribution on the optical properties. The deposition time of the sponges is 10 minutes (sponge A) and 1 hour (sponge $\mathrm{B}$ ). In both cases the decomposition temperature was $400{ }^{\circ} \mathrm{C}$ and the sponges were recrystallized at $800{ }^{\circ} \mathrm{C}$ during 1 hour. Thickness, mode (most repeated diameter value), and filling fraction are indicated for each sponge.

sponges show a clear transmission edge. Transmittance decreases by two orders of magnitude (from $50 \%$ to $0.5 \%$ ) in the wavelength region from 20 to $10 \mu \mathrm{m}$. Then, it increases slightly at shorter wavelengths. We explain this behaviour in terms of the light scattering properties of the single microspheres that make up the sponge, i.e. there is no multiple scattering. This happens because there exists two conditions: the geometry of the resonators is spherical, and the spheres are relatively isolated from each other, i.e. they support Mie modes. These are reasonable assumptions as we will see in the next section (4.3.2.2 The influence of the filling fraction). Nevertheless, in order to corroborate this hypothesis we calculated the contribution to the scattering cross-section $\left(\sigma_{s c a}\right)$ of all of the spheres composing the sponges according to the identified statistical distributions (assuming there is no multiple scattering). We considered again refractive index values given by Palik [9] and the result is plotted in figure 4.19 in dashed lines. While one sphere produces well defined deeps in transmission (figure 4.12), the scattering cross-section of the sponges results in smooth curves with a well defined edge that complements their transmittance spectra. Therefore, the decrease of transmission can be associated with an increase in Mie scattering. The edge in transmission corresponds to a transition zone, indicated in grey in figure 4.19 for sponge A, that separates two regimes where light scatters differently. The scattering process is Rayleigh type for wavelengths larger than those of the edge zone, and it is Mie type for shorter wavelengths.

The edge position of the transmittance spectra depends on the particular sphere size distribution. It is in good agreement with the calculated scattering cross section curves, i.e., the transmission edge and its comple- 


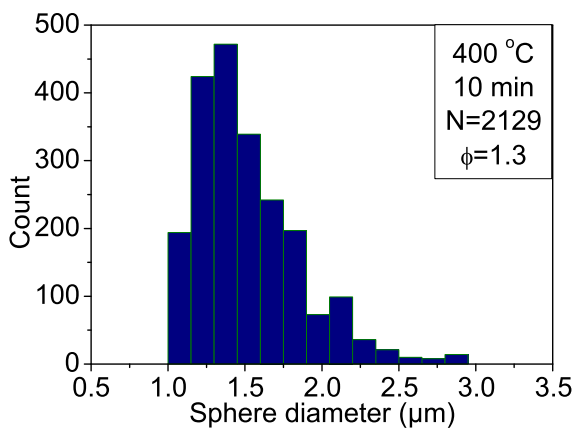

(a)

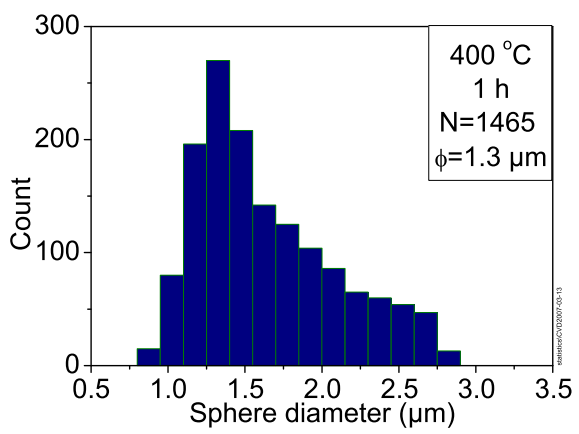

(b)

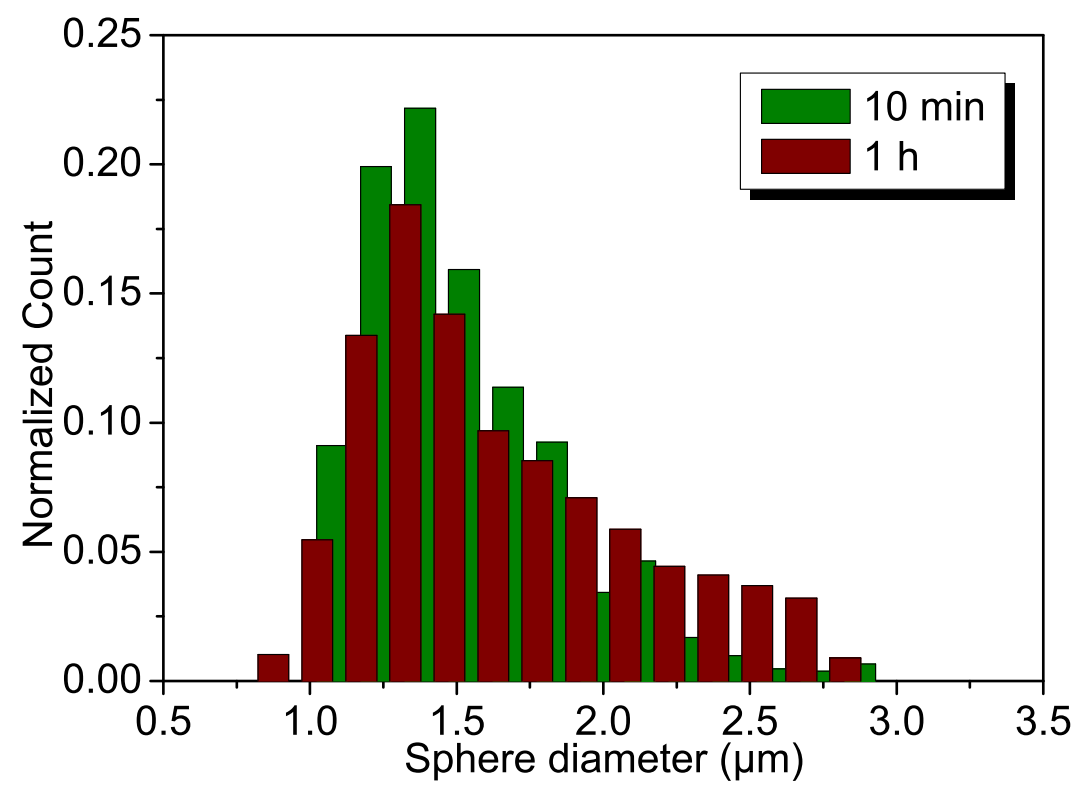

(c)

Figure 4.18: (a) and (b) sphere diameter distributions for photonic sponges $\mathrm{A}$ and $\mathrm{B}$ respectively (see table 4.3). The legends indicate deposition temperature, deposition time, number of spheres counted and the mode position. (c) Comparison between sponges A and B normalized.

mentary curve shift at the same time according to the sphere diameter distribution. The edge is given, in fact, by the flank of the first Mie mode $\mathrm{TE}_{1,1}$. Therefore, the bigger the spheres the larger the red-shift of the edge. This fact is clear when comparing the sphere size distributions of 


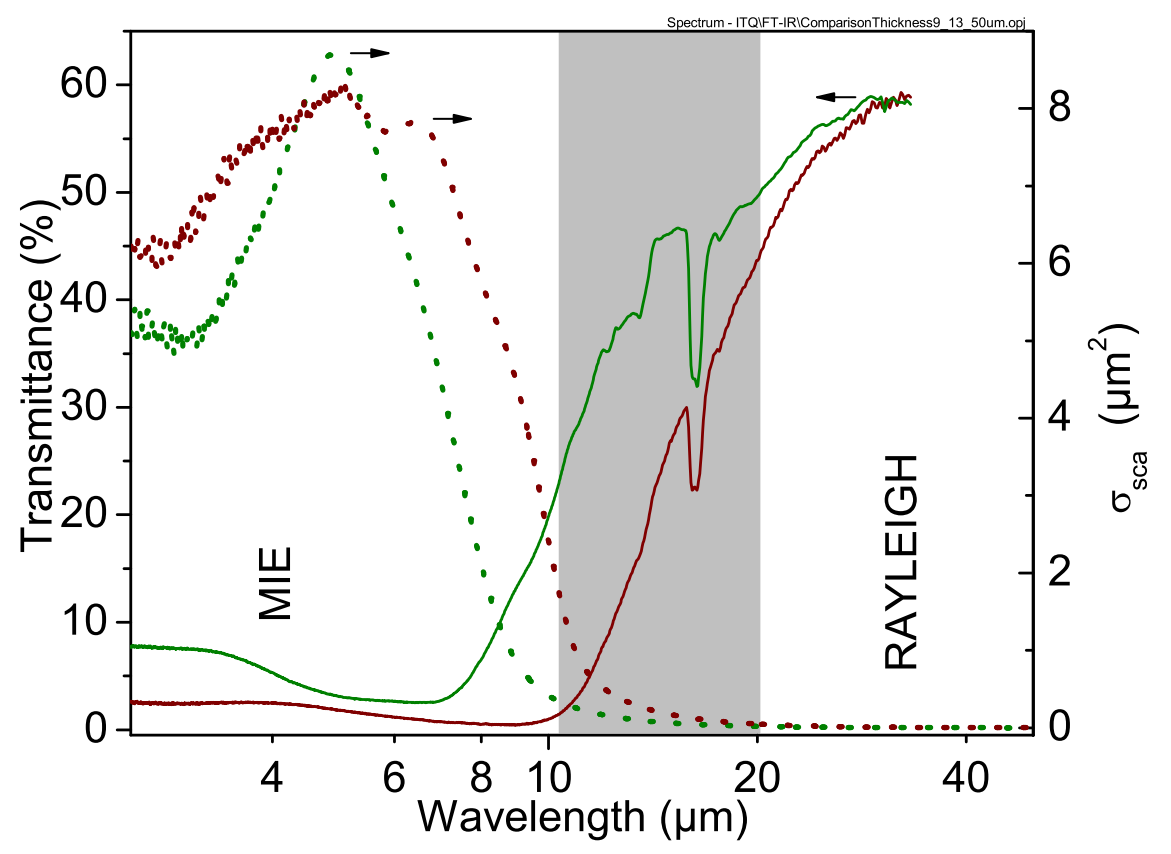

Figure 4.19: Optical properties of photonic sponges A (green) and B (red). The continuous lines correspond to the experimental transmittance measurements performed by FTIR spectroscopy. The dashed lines correspond to the calculation of the scattering cross section according to the sphere size distribution of the sponges (figure 4.18 (a) and (b)). The grey area indicates a transition zone where the type of scattering changes from Mie type to Rayleigh type for sponge B.

both sponges (figure 4.18(c)). They are relatively similar but sponge B has a larger body biased at larger sphere diameters, and consequently the edge in transmittance is placed at longer wavelengths than the edge of sponge A.

\subsubsection{The influence of the filling fraction}

As we mentioned in the previous section, the CVD method does not permit to modify easily some parameters of the photonic sponge such as thickness and filling fraction. Therefore, in order to be able to control the filling fraction $(\mathrm{FF})$ value as well as the sample thickness, we have chosen the method of embedding silicon colloids in a transparent medium 
for IR wavelengths. Such a medium is Potassium Bromide (KBr).

4.3.2.2.1 Optical properties of Potassium Bromide Potassium Bromide $(\mathrm{KBr})$ is an inorganic salt, optically transparent in the wavelength range of the EM spectrum from $0.4 \mu m$ to $50 \mu m$ [11]. It is used as an embedding medium to measure the optical properties of chemical species. KBr has no significant optical absorption lines in its high transmission region as shown in figure 4.20 (a). It's easy to handle but it must be kept in a dry environment due to its high solubility and its hygroscopic nature. Water absorbed in $\mathrm{KBr}$ cause a decrease in transmittance mainly for wavelengths from $3 \mu \mathrm{m}$ to $10 \mu \mathrm{m}$ (see figure 4.20(a)). Figure 4.20(b) illustrates the absorbance coefficient of water for infrared wavelengths with an intense peak around 3 micrometers.

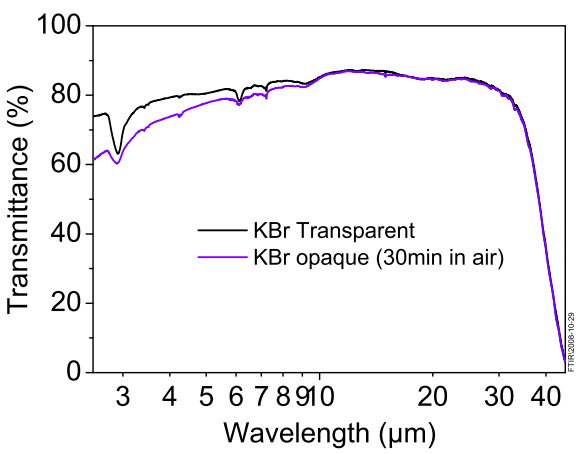

(a)

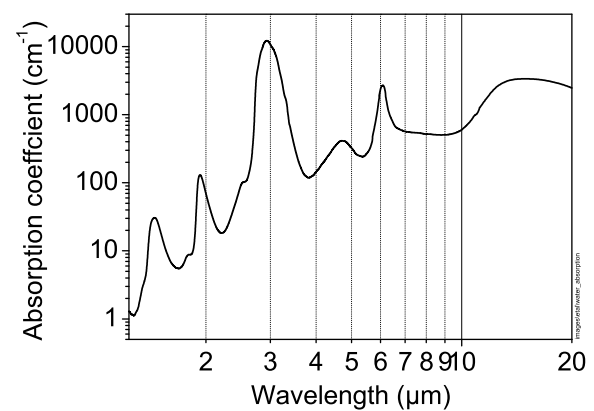

(b)

Figure 4.20: (a) Transmittance of a $\mathrm{KBr}$ disk in the infrared region. The disk is made in a $13 \mathrm{~mm}$ diameter die by using $200 \mathrm{mg}$ of $\mathrm{KBr}$. The disk thickness is approximately $566 \mu \mathrm{m}$. The black curve corresponds to the as prepared disk and the violet one corresponds to the same disk after having been exposed to air during 30 minutes. The deep at 3 micrometers is associated to water absorption (see (b)) and it is already present in the as-prepared sample. The exposure of the disk to air causes a decrease of transmittance in the region from the near infrared up to 10 micrometers. (b) Absorption spectrum of water [12].).

Another reason why $\mathrm{KBr}$ has been chosen as embedding medium is its low refractive index $(n=1.56)$ and the fact that it does not vary much for infrared wavelengths. Because of the high refractive index contrast between the silicon colloids (3.5) and the $\mathrm{KBr}$, the optical properties of the colloids should be barely modified by the embedding medium. This has allowed us performing spectroscopic measurements in the wavelength 
region from $2.5 \mu \mathrm{m}$ to $35 \mu \mathrm{m}$.

4.3.2.2.2 Sample preparation Silicon colloids deposited at $500{ }^{\circ} \mathrm{C}$ during 4 minutes and recrystallized in vacuum at $800{ }^{\circ} \mathrm{C}$ during 1 hour are used for the samples preparation. The colloids have a diameter distribution with the mode placed around $1.34 \mu \mathrm{m}$. We used short deposition times for avoiding the formation of big clusters. However, this way only a small amount of colloids is produced, and therefore preparing samples with a high filling fraction is very time consuming. As the vast majority of the deposited spheres are grouped in small clusters, they are gently separated by grinding means in an agate mortar with a small addition of $\mathrm{KBr}$. The progress of how well the clusters have been separated is supervised by an optical microscope (Nikon Eclipse LV100). The grinding process proceeds until the silicon spheres appear well separated or the largest cluster consist of 2-3 silicon colloids (this happens usually after 30-45 minutes of grinding). An example of how the spheres appear in $\mathrm{KBr}$ after the grinding process is depicted in figure 4.21.

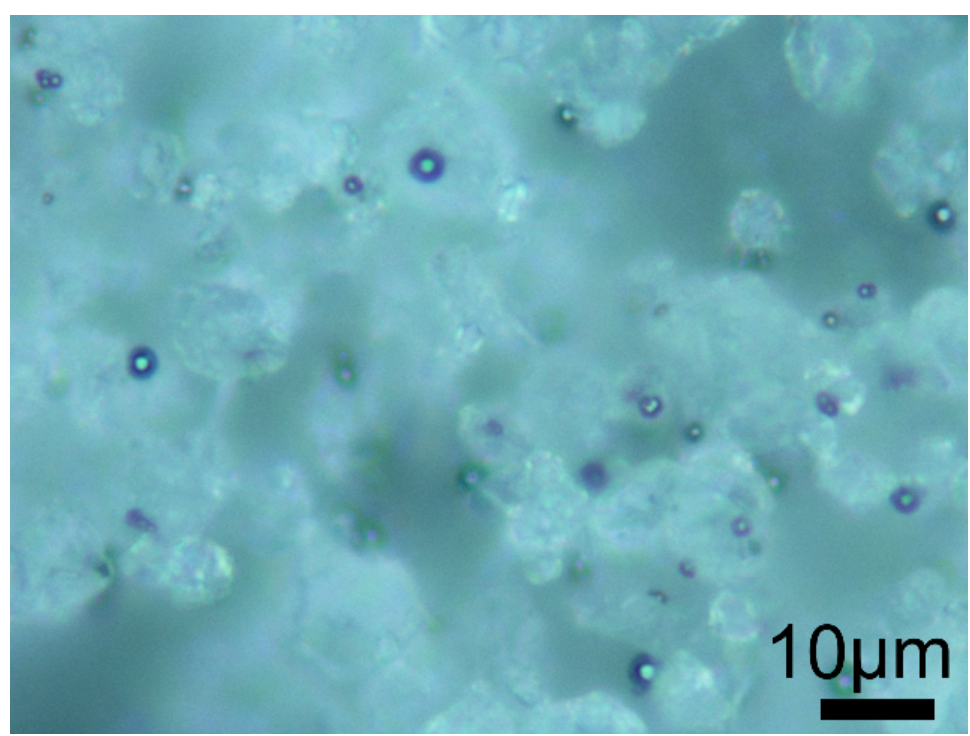

Figure 4.21: Silicon spheres embedded in KBr as seen by an optical microscope at 1000X magnification.

After the grinding process, the mixture of silicon colloids and $\mathrm{KBr}$ powder is heated at $130{ }^{\circ} \mathrm{C}$ during 1 hour to remove any residues of water. Then, in order to achieve the desired filling fraction, the appropriate amount of $\mathrm{KBr}$ is added to the mixture and the resulting mixture is 
compressed until becoming a disk by using a mold die with an hydraulic press. In order to explore the influence of the filling fraction in the optical properties of photonic sponges three different sample sets, each one containing samples of different thickness, were prepared: the first set corresponds to samples with a filling fraction (FF) (by volume) of $0.1 \%$, i.e. $(\mathrm{FF}=0.001)$, the second one corresponds to samples with a $\mathrm{FF}$ of $1 \%$, i.e. $(\mathrm{FF}=0.01)$ and the third set corresponds to samples with a $\mathrm{FF}$ of $30 \%$, i.e. $(\mathrm{FF}=0.3)$. Each $\mathrm{FF}$ gives an average distance between spheres. This distance has been calculated by using the "sphere in a box model" described in appendix A [13]. The obtained mean distances $(d)$ between spheres are summarized in table 4.4. They are written in micrometer units and also as the radio $d / a$ where $a$ is the average diameter of the spheres.

\begin{tabular}{|c|c|c|}
\hline Filling fraction $(\%)$ & Average distance in $\mu m$ between spheres & $d / a$ \\
\hline $0.001(0.1 \%)$ & 10.7 & 8 \\
$0.01(1 \%)$ & 4.7 & 3.5 \\
$0.30(30 \%)$ & 1.35 & 1 \\
\hline
\end{tabular}

Table 4.4: Filling fractions (FF) (by volume) of silicon colloids in KBr samples used for measuring the influence of the $\mathrm{FF}$ in the optical properties of photonic sponges. The average diameter $(a)$ of the spheres is $1.34 \mu \mathrm{m}$. The average distance $(d)$ between spheres corresponding to each FF has been calculated by using the "sphere in a box" model. This distance is expressed in micrometers (second column) and as the ratio $d / a$ (third column).

Those samples with a filling fraction of $0.1 \%$ and $1 \%$ are prepared by using a die mold of $13 \mathrm{~mm}$ in diameter, and those samples with a filling fraction of $30 \%$ are prepared by using a die mold of $5 \mathrm{~mm}$ in diameter. The reason for using a smaller die mold in the last case is the large amount of colloids that are necessary for preparing samples with such a high FF. This allows saving colloids because the samples are much smaller. A drawback is the smaller aperture of the disks.

4.3.2.2.3 The obtained spectra Figure 4.22 (a), (b) and (c) shows the IR transmission spectra of the prepared samples (see table 4.4) with filling fraction values of $0.001,0.01$, and 0.3 respectively. For each filling fraction, measurements corresponding to different sample thickness (indicated in the figure legends) are plotted. Generally speaking, for all of the $\mathrm{FF}$ values, the spectrum with the highest transmittance corresponds 
to the thinnest sample. For samples with $\mathrm{FF}=0.001$ (figure $4.22(\mathrm{a})$ )one can see that the thinnest sample (56 micrometers) shows a structureless spectrum that grows monotonically as the wavelength value increases. However, for the thicker samples transmission spectra show a step-like profile similar to those of as-grown photonic sponges (see figure 4.19), with the edge placed around 10 micrometers. This step-like behavior is present in all the studied samples with $\mathrm{FF}=0.01$ (figure $4.22(\mathrm{~b})$ ). However, it is absent for samples with $\mathrm{FF}=0.3$ (figure $4.22(\mathrm{c})$ ), where the transmittance curves show again a monotonically increase as the wavelength increases.

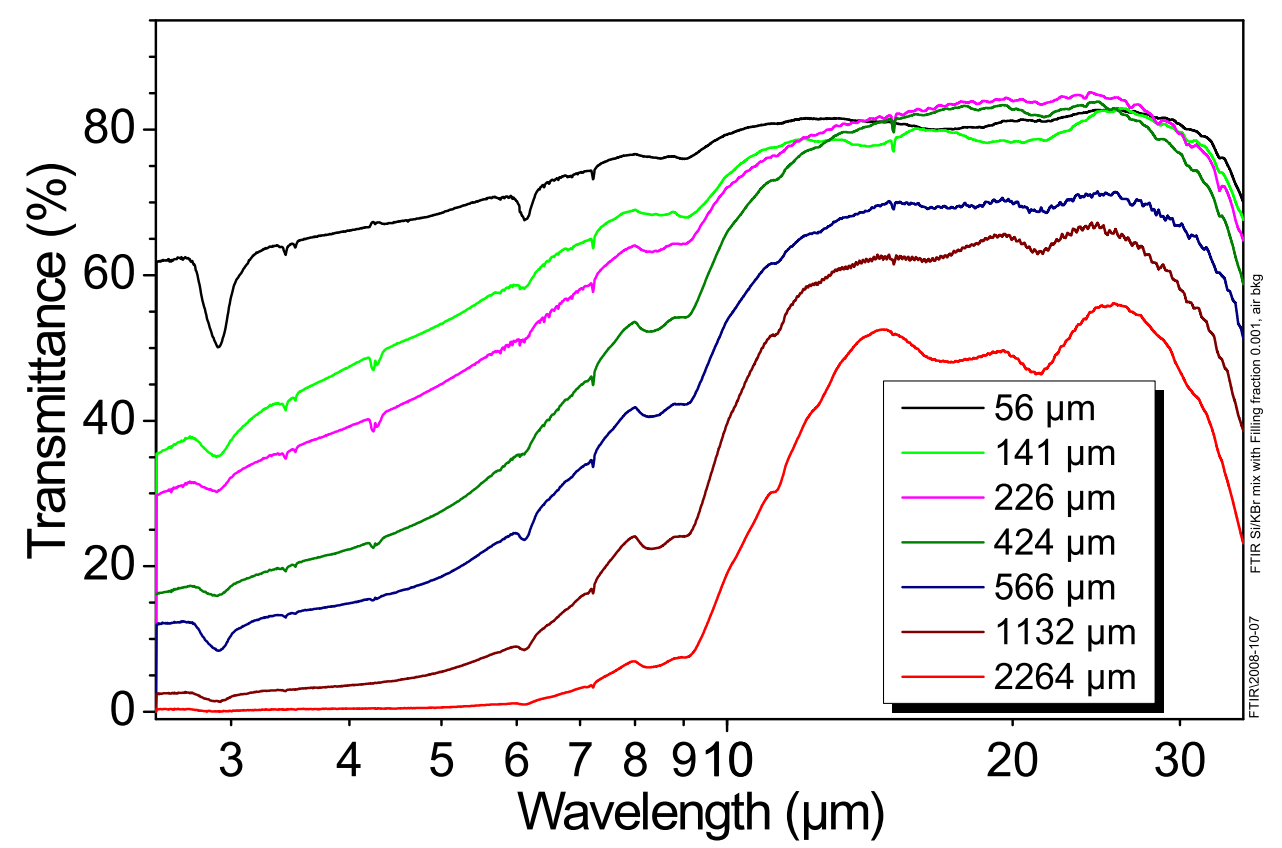

(a)

Figure 4.22: (a) Transmittance measurements of photonic sponges made of silicon colloids embedded in $\mathrm{KBr}$, for FF values of 0.001 (a), 0.01 (b), and 0.3 (c). For each FF, measurements were performed for different sample thickness whose values are indicated in the figure legends. Figure continue on next page... 


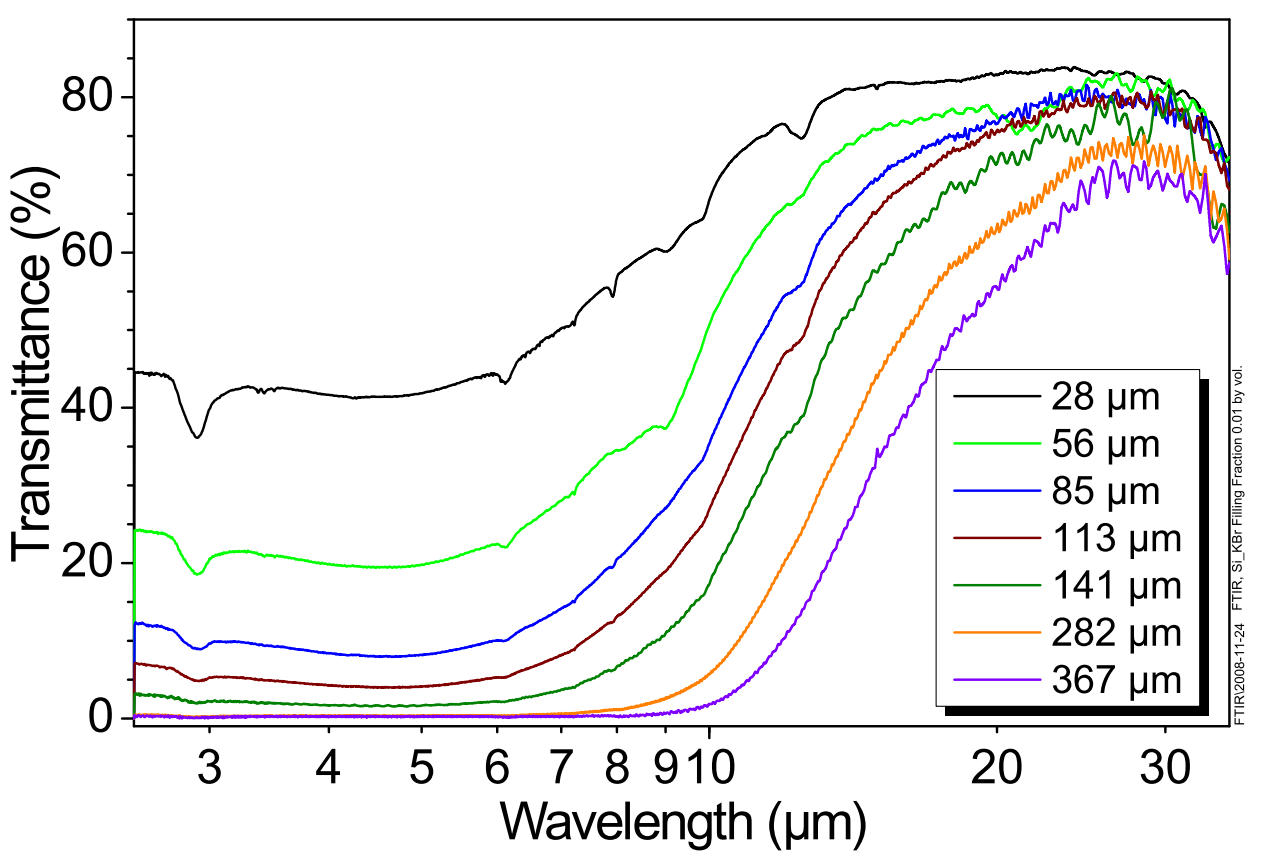

(b)

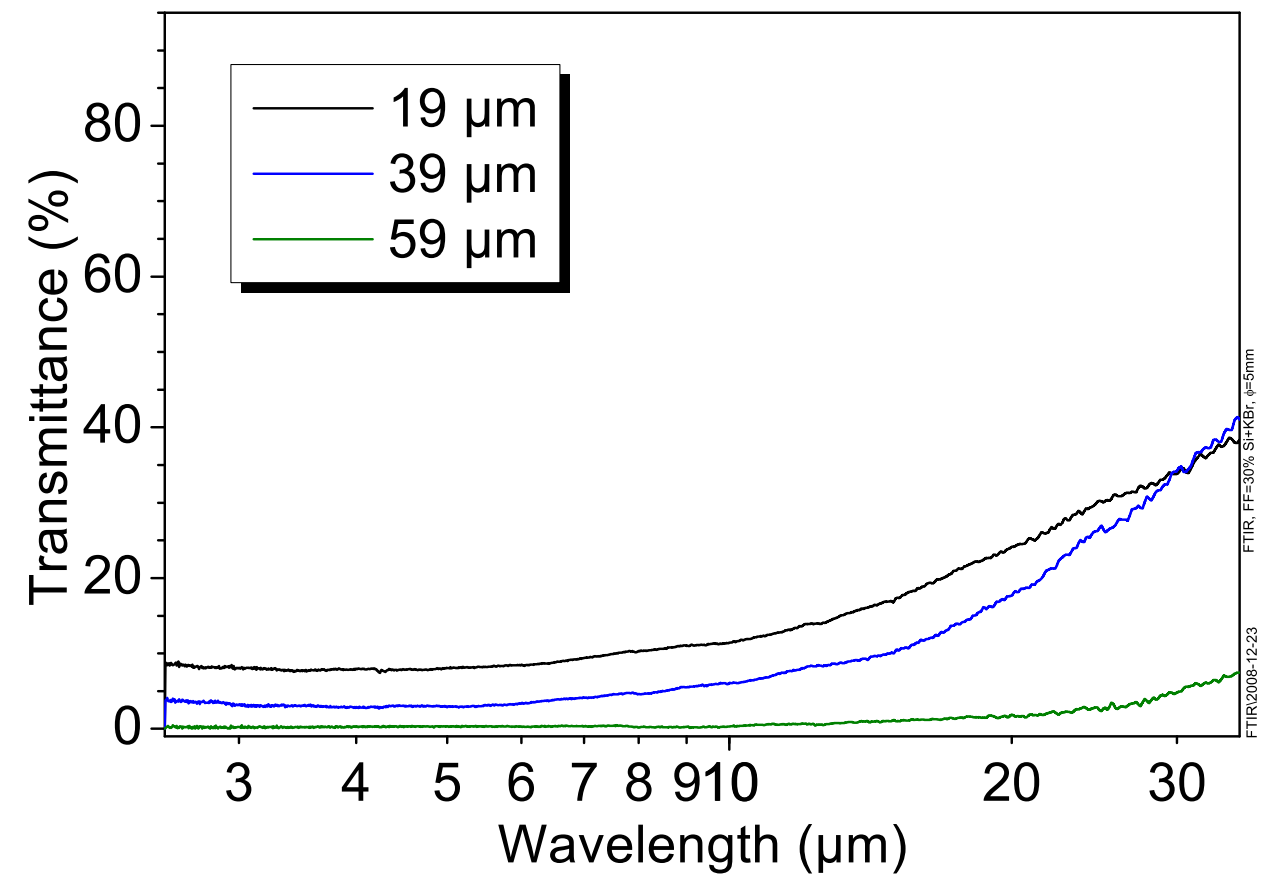

(c)

Continued Figure 4.22: Transmittance measurements of photonic sponges made of silicon colloids embedded in $\mathrm{KBr}$, for $\mathrm{FF}$ values of 0.01 (b), and 0.3 (c). 
We have not found a complete and quantitative explanation of the observed facts. This would require more different types of experiments and it constitutes the object of further work. Instead, we provide some hints that explain qualitatively the performed measurements. As mentioned in the previous section (4.3.2.1 The influence of the sphere size distribution), the step-like behaviour is attributed to the conjunction of two circumstances:

1. The geometry of the resonators composing the sponge is a defined one, namely a sphere. This makes the resonant modes have a defined position in the spectra. Although there are spheres of different sizes, the step in the transmittance signal corresponds to the upper wavelength limit beyond that, Mie resonances are absent; i.e.: this edge would correspond to the lower wavelength flank of the $\mathrm{TE}_{1,1}$ mode Mie resonance of the largest particles of the ensemble.

2. The spheres are relatively isolated from each other, i.e. the interparticle distance is larger than the sphere diameter. This allows Mie scattering of single particles to occur, i.e. there is no multiple scattering. As it is seen table 4.4, this condition is accomplished for $\mathrm{FF}=0.001(d / a=8)$ and $\mathrm{FF}=0.01(d / a=3.5)$ and also for the asgrown by CVD means photonic sponges (not specified in the table) where the $\mathrm{FF}=0.12(d / a=2.2 \mu \mathrm{m})$. All those samples with any of these conditions show the step like behaviour. However, this does not happen for samples where $\mathrm{FF}=0.3$ (see figure $4.22(\mathrm{c})$ ). Here, the inter-particle distance is similar to the average sphere diameter, and multiple scattering should occur. Therefore, the light does not "feel" the particles one by one but as a whole system, and thus the step-like behaviour is not present anymore (figure 4.22(c)).

The fact that there is a thickness onset for the step-like behaviour in samples with $\mathrm{FF}=0.001$ is presumably due to the fact that the "mean free path, l", i.e. the distance the light travels without having experienced any scattering event, is longer than the thickness values of the thinner samples. The $l$ value for $\mathrm{FF}=0.001$ could be around 226 micrometers, which is the thickness value of the thinnest sample that shows the steplike behaviour. However for samples with $\mathrm{FF}=0.01$, the $l$ value should be smaller than 28 micrometers because in this case no onset was observed and the step-like behaviour was already present in the thinnest measured sample with such a thickness value. 


\subsection{Conclusions}

In this chapter we have analyzed the optical properties of single silicon colloids and photonic sponges. The following conclusions can be derived:

- Silicon colloids work as photonic microcavities with well defined resonating Mie modes. The agreement between experimental transmittance and the calculated one by Mie theory corroborate that the colloids are in fact made of silicon and their structural properties of sphericity and surface smoothness remain in good condition.

- The quality factors of the observed Mie resonances in single silicon colloids are in general lower than the expected theoretical values. This is attributed to residual absorbance and it is most probably provoked by the existence of defects near the electronic gap of silicon. These defects may be related to different causes like defects in the crystalline structure, some contamination, etc. They are objects of future research.

- Photonic sponges, formed by disordered networks of silicon colloids, show a transition region between two types of scattering, Rayleigh and Mie. This phenomenon can be explained in terms of Mie scattering of single colloidal particles and it occurs whenever the interparticle distance is larger than the average sphere diameter. The spectral position of the transition edge depends on the particular sphere diameter distribution of the sponge. 


\section{Bibliography}

[1] Bahaa E. A. Saleh and Malvin Carl Teich. Fundamentals of Photonics. Wiley-Interscience, New Jersey, USA, 2007.

[2] Lord Rayleigh. Scientific Papers, 5:617, 1912.

[3] Mie Gustav. "Beiträge zur Optik trüber Medien, speziell kolloidaler Metallösungen". Annalen der Physik, 330(3):377-445, 1908.

[4] Craig F. Bohren and Donald R. Huffman. Absorption and Scattering of Light by Small Particles. Wiley-Interscience, New York, 1998.

[5] Julius Adams Stratton. Electromagnetic Theory. Kluwer Academic Publishers, New York, 1941.

[6] H. C. van de Hulst. Light Scattering by small particles. Dover Publications, New York, 1981.

[7] Max Born and Emil Wolf. Principles of Optics: Electromagnetic Theory of Propagation, Interference and Diffraction of Light. Cambridge University Press, United Kingdom, Cambridge, 1997.

[8] P. W. Barber and S. C. Hill. Light Scattering By Particles: Computational Methods. World Scientific, Singapore, 1990.

[9] E Palik. Handbook Of Optical Constants Of Solids. Academic Press, New York, 1985.

[10] Landolt-Brnstein. Numerical Data And Functional Relationships In Science And Technology, Group III. Springer-Verlag, Berlin, 1982.

[11] M. A. Ford and G. R. Wilkinson. "The preparation and properties of pressed alkali halide disks with special reference to their use in spectroscopy". Journal of Scientific Instruments, 39(9):338-341, 1954.

[12] David M. Wieliczka, Shengshan Weng, and Marvin R. Querry. "Wedge shaped cell for highly absorbent liquids: infrared optical constants of water". Appl. Opt., 28(9):1714-1719, 1989.

[13] Dionys Krasnokutsky. "Estimation of Distance Between Particles of Powder in Well-Dispersed Sample for Particle Size Analysis". The General Science Journal, Mars 20, 2007. 


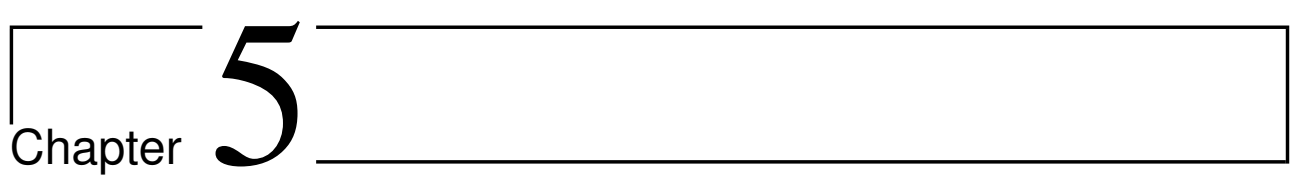

\section{General conclusions}

\subsection{Conclusions}

In this thesis, we have shown a method to obtain a novel material: silicon colloids. They consist of spherical particles with size from 0.5 to 3.5 micrometers, than can be made of amorphous or poly-crystalline silicon. We have investigated their structural and optical properties.

Silicon colloids have good structural properties of sphericity and surface smoothness, and this allows them work as optical microcavities with well defined Mie modes in the near infrared range. Moreover, because of the high refractive index of silicon, low index modes like for instance $\mathrm{TE}_{1,1}$ or $\mathrm{TM}_{1,1}$, etc have been predicted and measured.

We have also investigated the optical properties of photonic sponges that are formed by disordered arrangements of silicon colloids of different sizes. They show a particular optical behaviour consisting of a step-like profile with two distinct spectral regions where light scatters differently: Rayleigh type and Mie type. The key factors that explain this behaviour are the spherical geometry of the scatterers and a filling fraction value small enough for avoiding multiple scattering between them.

\subsection{Towards future}

This thesis constitutes the starting point of studying of a novel material, silicon colloids. Therefore, many questions related to different aspects of the topic have arisen during this research. Some of the most important questions are the synthesis mechanisms, the reason why the experimental 
quality factor of the modes is always lower than the theoretical values, the light propagation inside the photonic sponges, etc. In spite of all unanswered questions, we believe silicon colloids hold a great potential to realize novel functional photonic devices, like for instance one sphere sensor, detector or laser. On another hand, silicon colloids could also be used in fields such as cosmetics and functional medicine. In any case, one main problem must be solved: to improve the quality of the silicon colloids. 
Chapter

\section{List of publications}

\subsection{Scientific Articles in International Jour- nals}

[1] Roberto Fenollosa, Fernando Ramiro-Manzano, Michal Tymczenko, and Francisco Meseguer. "Porous silicon microspheres: synthesis, characterization and application to photonic microcavities". Journal of Material Chemistry, Accepted, 2010.

[2] Roberto Fenollosa, Francisco Meseguer, and Michal Tymczenko. "Silicon Colloids: From Microcavities to Photonic Sponges". Advanced Materials, 20(1):95-98, 2008.

[3] Michael Tymczenko, Lluis F. Marsal, Trifon Trifonov, Isabelle Rodriguez, Fernando Ramiro-Manzano, Josep Pallares, Angel Rodriguez, Ramon Alcubilla, and Francisco Meseguer. "Colloidal Crystal Wires". Advanced Materials, 20(12):2315-2318, 2008.

[4] Yoshinori Tanaka, Michal Tymczenko, Takashi Asano, and Susumu Noda. "Fabrication of Two-Dimensional Photonic Crystal Slab Point-Defect Cavity Employing Local Three-Dimensional Structures". Japanese Journal of Applied Physics, 45(8A):6096-6102, 2006. 


\subsection{Cover Images in International Journals}

Inside Front Cover page

Journal: Advanced Materials

Volume: 20, Issue: 12, Year: 2008

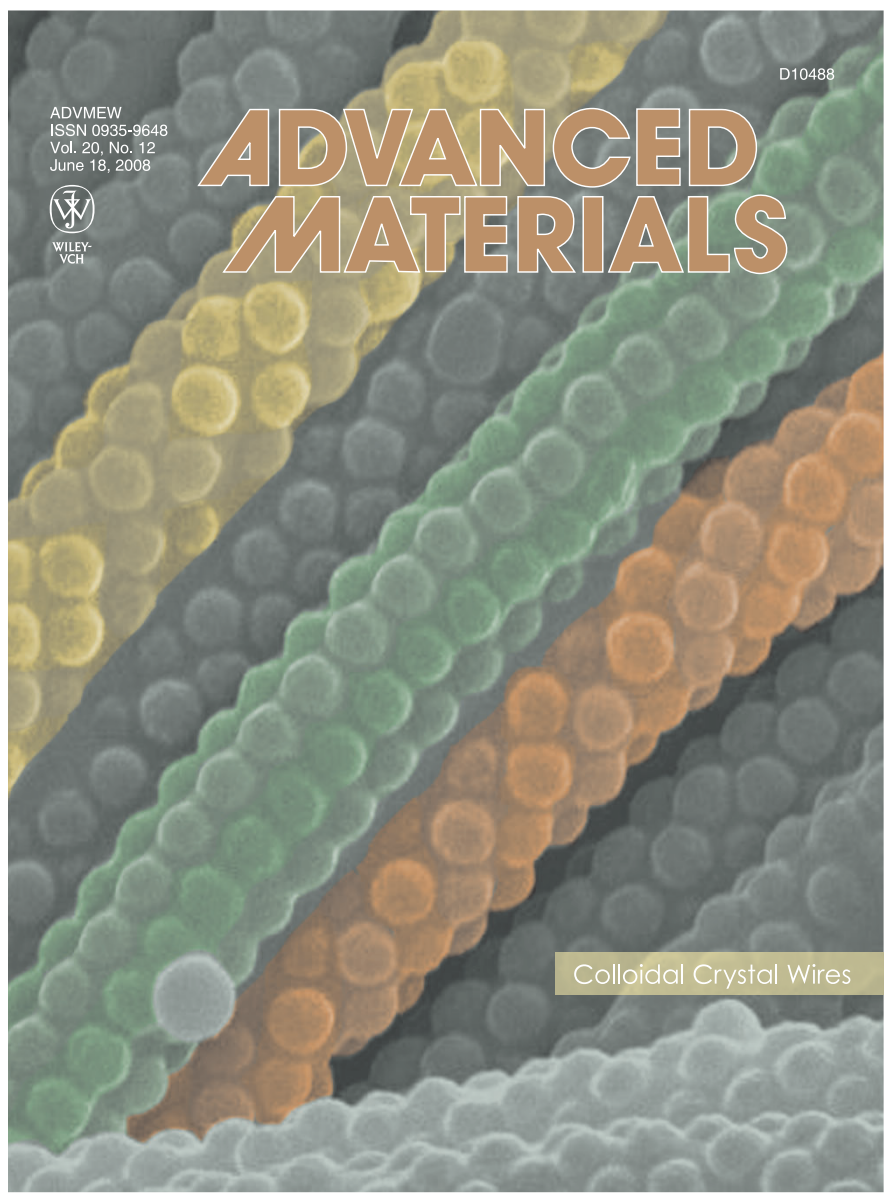

Figure 6.1: Advanced Materials inside front cover. 


\subsection{International Conferences and Symposia}

[1] Roberto Fenollosa, Francisco Meseguer, and Michal Tymczenko. "Optical microcavities and photonic sponges based on silicon spheres". In poster, The 1st "Conferencia Espaola de Nanofotonica", April 2-4, Tarragona, 2008.

[2] Michael Tymczenko, Lluis F. Marsal, Trifon Trifonov, Isabelle Rodriguez, Fernando Ramiro-Manzano, Josep Pallares, Angel Rodriguez, Ramon Alcubilla, and Francisco Meseguer. "Wiring Order and Chirality in Colloidal Crystal Fibres". In poster, The 1st "Conferencia Espaola de Nanofotonica", April 2-4, Tarragona, Spain, 2008.

[3] Michael Tymczenko, Lluis F. Marsal, Trifon Trifonov, Isabelle Rodriguez, Fernando Ramiro-Manzano, Josep Pallares, Angel Rodriguez, Ramon Alcubilla, and Francisco Meseguer. "Wiring Order and Chirality in Colloidal Crystal Fibres". In poster, The 1st European Topical Meeting on Nanophotonics and Metamaterials, 8-11 January, Seefeld, Austria, 2008.

[4] Roberto Fenollosa, Francisco Meseguer, and Michal Tymczenko. "Silicon colloids". In poster, The 2nd European Topical Meeting on Nanophotonics and Metamaterials, 5 - 8 January, Seefeld, Austria, 2008 .

\subsection{Domestic Conferences and Symposia}

[1] Fernando Ramiro-Manzano, Roberto Fenollosa, Francisco Meseguer, and Michal Tymczenko. "Photoluminescence and aging effects in photonic microcavities based on porous silicon microspheres". In poster, 2nd Annual NanoLight meeting, March 9, Universitat Politcnica de Valencia, Valencia, 2010.

[2] Michael Tymczenko, Lluis F. Marsal, Trifon Trifonov, Isabelle Rodriguez, Fernando Ramiro-Manzano, Josep Pallares, Angel Rodriguez, Ramon Alcubilla, and Francisco Meseguer. "Wiring Order 
and Chirality in Colloidal Crystal Fibres". In poster, 1st Annual NanoLight meeting, February 23, ICFO, Castelldefels, Barcelona, 2009 .

[3] Roberto Fenollosa, Francisco Meseguer, and Michal Tymczenko. "Silicon colloids". In poster, 1st Annual NanoLight meeting, February 23, ICFO, Castelldefels, Barcelona, 2009.

[4] Roberto Fenollosa, Francisco Meseguer, and Michal Tymczenko. "Optical properties of Silicon Colloids solid suspensions". In poster, 1st Annual NanoLight meeting, February 23, ICFO, Castelldefels, Barcelona, 2009.

\subsection{Patents}

Title: Microesferas de silicio y esponjas fotónicas, procedimiento de producción y sus aplicaciones en la fabricación de dispositivos fotónicos. Authors: R. Fenollosa, F. Meseguer, M. Tymczenko Patent number: P200701681

Origin country and date: Spain, Madrid, June 2007

Title: Silicon microspheres and photonic sponges, production procedure and their applications.

Authors: R. Fenollosa, F. Meseguer, M. Tymczenko

Patent number: 11/984870

Origin country and date: USA, June 2007 


\section{List of Figures}

1.1 Laser cavity and FSR . . . . . . . . . . . . . . 15

1.2 Fabry-Pérot semiconductor laser . . . . . . . . . . . 16

1.3 Example of 1D, 2D and 3D PC . . . . . . . . 17

1.4 Schematic of a 2D Photonic Crystal . . . . . . . . . 18

1.5 Different types of whispering gallery mode cavities . . . . 19

2.1 CVD setup . . . . . . . . . . . . . 26

2.2 Tubular oven used to fabricate silicon colloids . . . . . . 28

2.3 Silicon colloids obtained by the hot wall thermal CVD process . . . . . . . . . . . . . . . . 29

2.4 Simplified schematics of disilane dissociation . . . . . 30

3.1 Histograms for sphere size distribution . . . . . . . . 35

3.2 Spheres found by MATLab script in a SEM image . . . . 36

3.3 Graph of mode versus decomposition temperature . . . . 37

3.4 Histogram comparison $500^{\circ} \mathrm{C} 4 \& 60 \mathrm{~min} \ldots . . . . . .38$

3.5 Convection current inside reactor . . . . . . . . . . 39

3.6 Profile of non uniform silicon colloid layer . . . . . . . . 40

3.7 XRD diagrams . . . . . . . . . . . . . . . . 42

3.7 XRD diagrams . . . . . . . . . . . . . . . . 43

3.8 Raman spectra comparison . . . . . . . . . . . . . . . . . 45

3.9 HRTEM images of silicon colloids . . . . . . . . . . 46

3.9 HRTEM images of silicon colloids . . . . . . . . . . 47

3.10 Effect of annealing treatment . . . . . . . . . . . . 49

3.11 Set of Si-H vibrational modes . . . . . . . . . . 50

3.12 Mass spectroscopy of silicon colloids . . . . . . . . . 51

3.13 Sphere surface analyzed by SEM . . . . . . . . . . . 52 
3.14 Colloid surface analyzed by AFM . . . . . . . . . 53

4.1 Scattering of light by a particle . . . . . . . . . . . 59

4.2 Resonant mode of a silicon sphere . . . . . . . . . . 60

4.3 Spherical coordinate system . . . . . . . . . . 61

4.4 TE modes . . . . . . . . . . . . . . . . . 65

4.5 TM modes . . . . . . . . . . . . . . . . . . 66

4.6 Calculated $\sigma_{s c a}$ and $Q_{s c a} \ldots \ldots \ldots \ldots$. . . . . . . . 67

4.7 Calculated Scattering cross section for low and high index materials . . . . . . . . . . . . . . . 69

4.8 Calculated Scattering cross section for low and high index materials . . . . . . . . . . . . . . . 69

4.9 Setup for measuring the transmittance for single silicon colloid. . . . . . . . . . . . . . . 71

4.10 Relation between pinhole diameter . . . . . . . . . 72

4.11 Refractive index for crystalline silicon Palik . . . . . . . 73

4.12 Fit of the transmittance spectra for single silicon colloid. $\quad 75$

4.13 Fit of the transmittance spectra for single silicon colloid. $\quad 76$

4.14 Fit of the transmittance spectra for single silicon colloid. $\quad 77$

4.15 Fit of the transmittance spectra for single silicon colloid. 78

4.16 Photonic sponge. . . . . . . . . . . . . . . . 81

4.17 Transmittance of silicon wafer . . . . . . . . . 83

4.18 Histogram for sphere diameter distribution $400{ }^{\circ} \mathrm{C}$. . . $\quad 85$

4.19 Experimental transmittance of a photonic sponge . . . 86

4.20 Transmittance of $\mathrm{KBr}$ disk . . . . . . . . . . . . 87

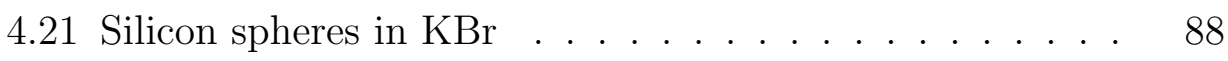

4.22 FTIR of disks with filling fraction of $0.1 \% \ldots \ldots$

4.22 FTIR of disks with filling fraction of $0.1 \% \ldots 91$

6.1 Advanced Materials inside front cover . . . . . . . . . 98

A.1 Sphere in the box model . . . . . . . . . . . 105

B.1 D parameter . . . . . . . . . . . . . . . . . 108

B.2 Showcase of results of other groups . . . . . . . . 109

B.3 Macroporus silicon membrane . . . . . . . . . . 110

B.4 CCW setup . . . . . . . . . . . . . . . 112

B.5 CCW steps . . . . . . . . . . . . . . . . . 113

B.6 Failed CCW . . . . . . . . . . . . . . . . . . 114

B.7 Macroporus silicon membrane infiltrated with latex spheres 115 
B.8 CCW fibers . . . . . . . . . . . . . . . . . . . 116

B.9 CCW versus $D$ factor parameter . . . . . . . . 118 


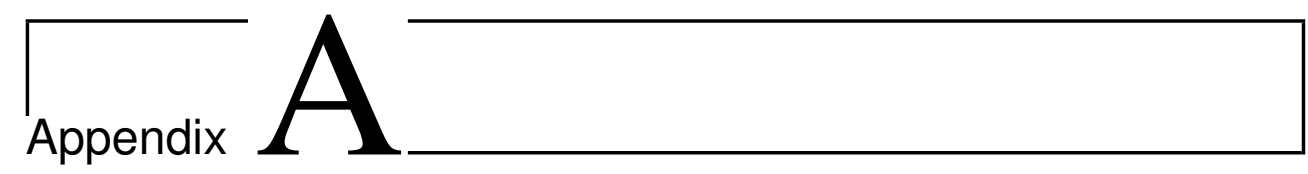

\section{Sphere in the box model}

Let us imagine that in a well dispersed sample each particle is situated in a box (cube) as illustrated in figure A.1. The whole sample consists of $n$ equal cubes with an edge length $a$, where $n$ is the number of particles.

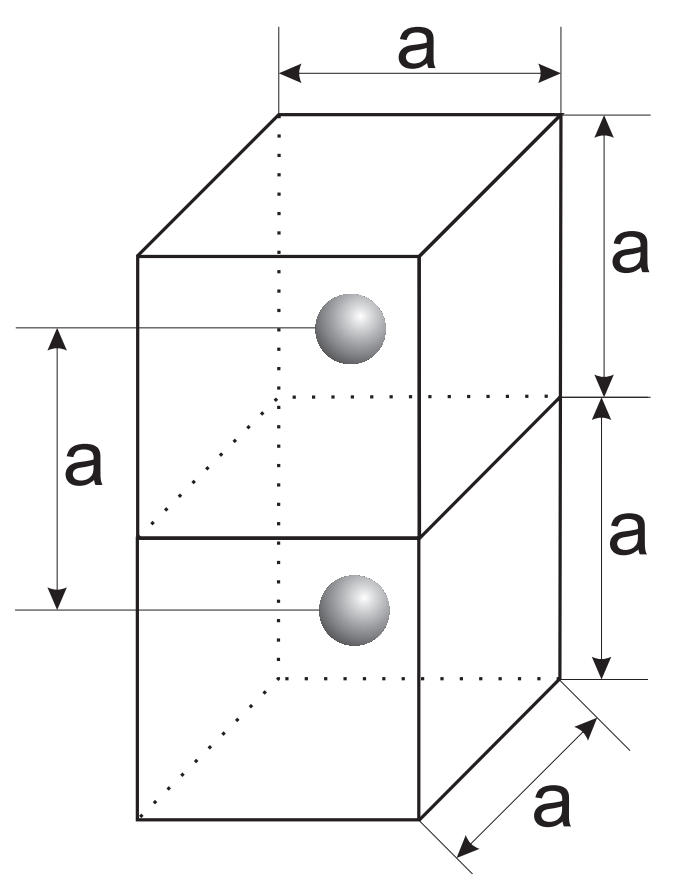

Figure A.1: Sphere in the box model. Two equal cubes with length $a$. The volume of one cube is $a^{3}$. The distance between the spheres is $a$.

After making the assumption that each particle is situated in the 
middle of the each cube, it can be seen that the shortest distance between the particles is $a$. We know that the volume of one cube can be calculated by equation (A.1):

$$
V_{\text {cube }}=a^{3} .
$$

We weight the particles and the embedding medium (KBr) separately. Then, assuming an average sphere diameter, the number of particles, and the number of cubes $(n)$ can be estimated by dividing the weight of the particles by the weight of the average particle. The volume occupied by the particles and the embedding medium $\left(V_{t o t}\right)$ can be calculated by using the density of silicon and $\mathrm{KBr}$. Now, $V_{\text {tot }}$ is divided by $n$ to obtain the $V_{\text {cube }}$, and finally $a$, the distance between particles, is given by equation (A.2):

$$
a=\sqrt[3]{V_{\text {cube }}} .
$$




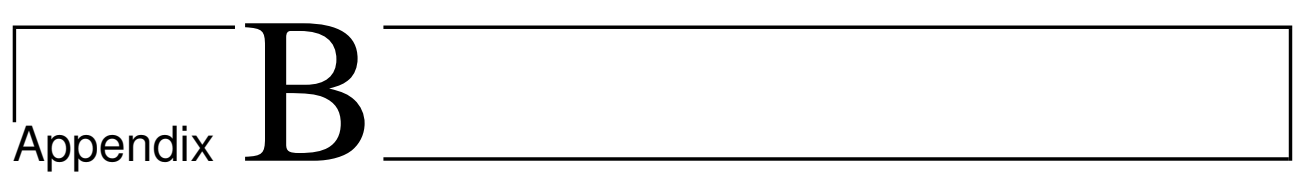

\section{Colloidal Crystal Wires}

\section{B.1 Introduction}

Colloidal Crystal Wires (CCW) have been extensively applied in many areas of science and technology. They can be used as a model of crystal order and phase transition processes of atoms and molecules [1-3]. As the size of colloidal particles, of customary use, are in the micrometer or sub-micrometer regimes, they produce strong diffraction effects in the optical region. This effect has been, and is still being, extensively applied to several fields of research such as photonic crystals [4, 5] and sensors [6]. Diffraction effects are dependent on the type of crystal order, that is, on the point group symmetry by which colloids are ordered. Hard-sphere colloidal particles order in closedpacked assemblies with preference for the face-centered cubic (fcc) symmetry $[7,8]$. However, the crystallization process of colloidal suspensions in confined geometries is very different from that in bulk systems. So far, most of the work in this area has focused on colloidal crystal thin films, which corresponds to one-dimensional confinement, where different types of close-packed and non-closed-packed facets appear [9-15]. In the case of two-dimensional confinement, colloidal crystal wires (CCW) are obtained. The important parameter to be considered here is the ratio between the confinement size and particle diameter, which we call the confinement ratio $D$ as illustrated in figure B.1. 


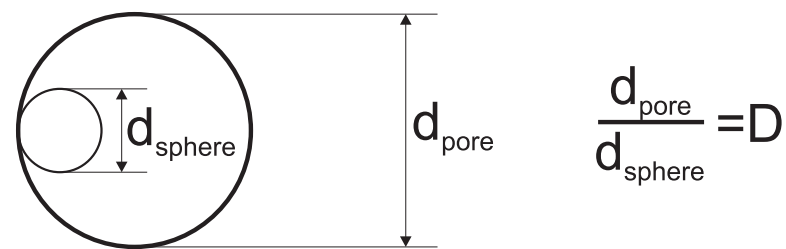

Figure B.1: The $D$ parameter is defined as the ration between pore diameter and sphere diameter.

There are two limiting cases depending on the $D$ value. When $D>1$, particles arrange themselves in a wide range of close-packed structures depending of the geometric shape of the template [16-18]. For example, squarelike colloidal fibers were obtained with lithographically prepared open channels templates [19-21], as well as with square-shaped optical fibers [22]. Also, a colloidal crystal capillary column has recently been reported [23]. New colloidal crystal arrangements appear when the patterned substrates have a lateral dimension similar to the particle size, that is, when $D=1$. Then, non-close-packed structures are favored [24] and, in some cases, helical ordering is induced [25](figure B.2(a)). But, most of all above cases do not show a pure 2D confinement, because they concern particle ordering into open patterned templates. Spheres distribute themselves in wirelike manner as has been modeled by Ericksson [26] and Picket et al. [27], through a hard-sphere packing model, and then shown for samples with beads in the millimeter range [28](figure B.2(b)). These authors have demonstrated that, depending on the $D$ factor, particles pack themselves in a manifold variety of pattern configurations. Owing to their potential applications, colloidal crystals fibres have raised great interest. Also, they can be used as a model for the formation of chiral molecules as liquid crystals [29] or DNA [30]. Following these seminal works, some groups have reported experimental results on the arrangement of spherical particles into capillary pores in length scale ranging from 100 micrometers [31](figure B.2(c)). Other groups have used carbon nanotubes as the template and used fullerenes as the filling spheres [32](figure B.2(d)). Recently, another approach that concerns the infiltration of particles into porous membranes made of alumina [33, 34](figure B.2(e)) or silicon [35, 36](figure B.2(f)) has been reported where free-standing $\mathrm{CCW}$ were obtained. Although in most cases random packing geometries were observed, some fibers show helical twist, a fingerprint of chirality. However, no systematic experimental 
study of the influence of the $D$ parameter on the particle order has been published.

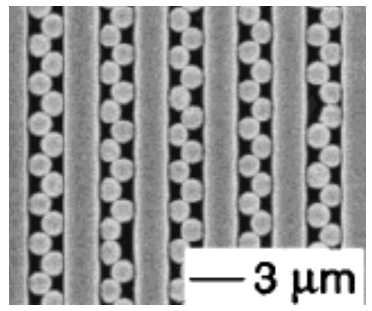

(a)

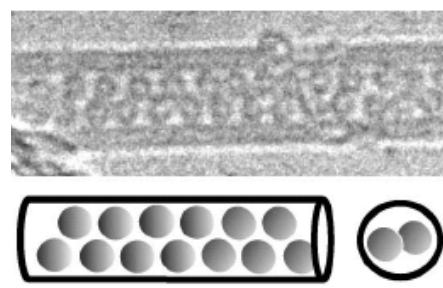

(d)

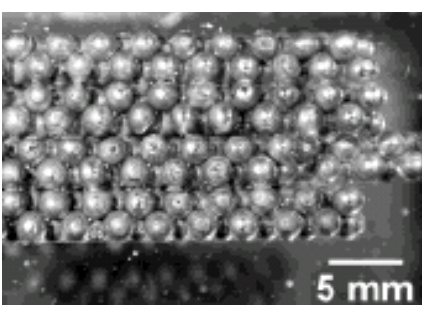

(b)

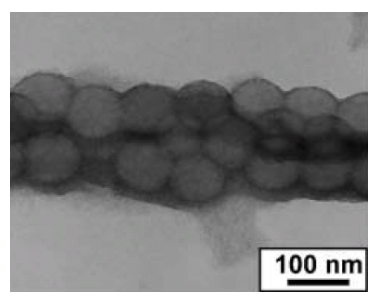

(e)

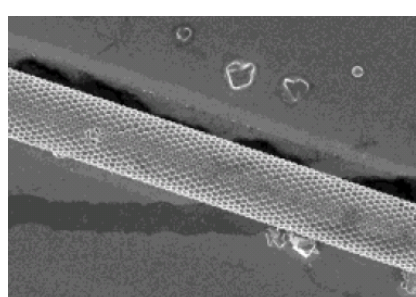

(c)

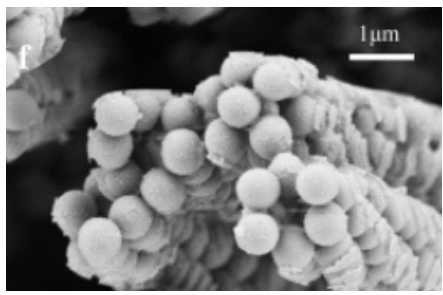

(f)

Figure B.2: Showcase of results of other groups. (a) Polystyrene colloids in 2D silicon template (Yin et al). (b) Millimeter-scale spherical beads using hierarchical self-assembly (Wu et al). (c) Silica microcapillary template with polystyrene particles (Moon et al). (d) Fullerenes in carbon nanotubes (Khlobystov et al). (e) Titania/polymer composite nanotubings with latex particles (Huang et al). (f) Free standing CCW made of silica spheres (Li et al).

With our approach by utilizing macroporus silicon membrane as a quasi cylindrical template and latex spheres of various diameters we can perform a systematic experimental study of particle ordering. By using PDMS as a binder for the latex spheres, results not previously obtained could led to verification of theoretical models [27].

Macroporus silicon membrane depicted in figure B.3 prepared by the light-assisted electrochemical etching is used as the template for the colloidal crystal wires. The electrochemical etching method [37, 38] produces very long pores with almost vertical walls. The entrance pore have a square shape and gradually change the shape to circular. The pore size decreases gradually in size from a nominal value at the pore entrance to a value around $50 \%$ or less pore exit. In numbers the pore entrance have $4 \mu \mathrm{m}$ across and the exit pore is round, $2 \mu \mathrm{m}$ in diameter as shown in figure B.3(a,b). Thanks to the quasi-cylindrical shape of the pore, and 
also owing to the smooth change of the pore diameter, it is possible to study how the CCWs arrange themselves in an almost perfect geometry for different values of the $D$ parameter, ranging from 1 to 3 .

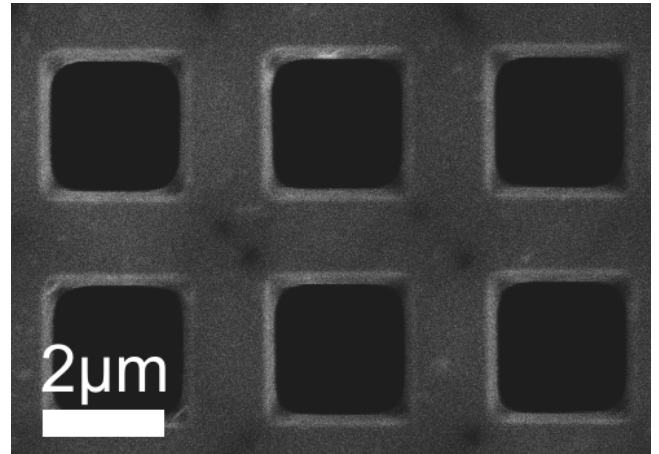

(a)

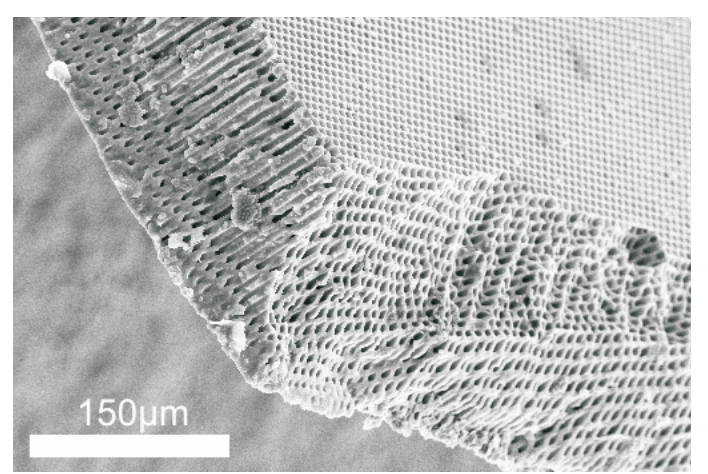

(c)

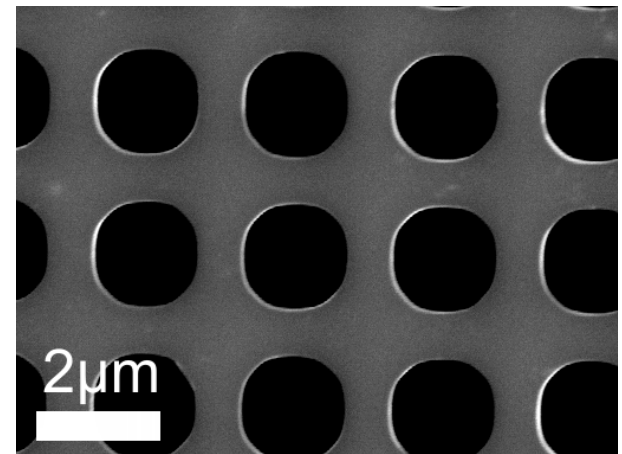

(b)

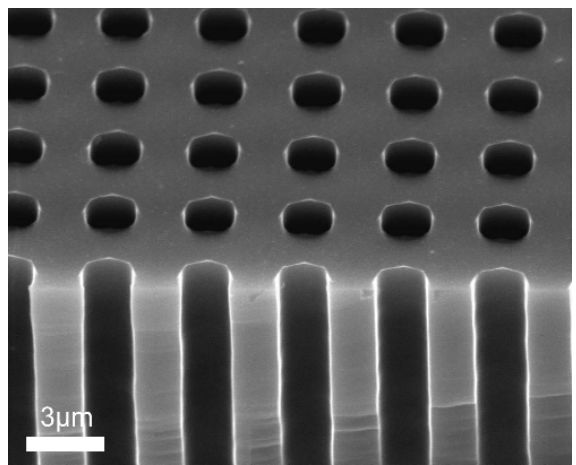

(d)

Figure B.3: Macroporus silicon membrane. (a) Square like entrance pore. (b) Round exit pores. (c) Edge of macroporous silicon membrane. (d) Cut through the membrane showing the almost vertical walls of the pores. 


\section{B.2 Experimental setup}

\section{B.2.1 Fabrication of macroporous silicon membrane}

Silicon membranes were kindly supplied by the Universitat Rovira i Virgili (LL. Marsal group) and Universitat Politćnica de Catalunya (R. Alcubilla group). Silicon membrane was prepared by light-assisted electrochemical etching [37-39] and post-processing. The starting material was n-type $\langle 100\rangle$ silicon with a resistivity of $2-6 \Omega \mathrm{cm}$. The front side of the wafers was patterned with inverted pyramid-shaped pits by oxidation, photolithography, and subsequent tetramethyl ammonium hydroxide (TMAH) etching. The pattern consisted of $3 \times 3 \mathrm{~mm}^{2}$ inverted pyramids arranged in a square lattice with a period of $4 \mu \mathrm{m}$. These inverted pyramids act as nucleation sites for the ordered pore growth. A transparent ohmic contact was made by implanting an $\mathrm{n}+$-layer on the wafer backside. The wafers were anodized in $5 \mathrm{wt} \%$ aqueous hydrofluoric acid (HF) electrolyte at $15{ }^{\circ} \mathrm{C}$ and under backside illumination. $0.1 \mathrm{mM}$ of the TritonX-100 non-ionic surfactant was added to the electrolyte as a wetting agent. The current density was kept at $5 \mathrm{~mA} \mathrm{~cm}{ }^{-2}$ during the etching by controlling the backside illumination intensity. The pore diameter was proportional to the number of positive-charge carriers that were collected by the pore tip, which in turn depended on the intensity of backside illumination. Keeping the etching current constant was needed to get straight pores. In the experimental conditions described above, $150 \mu \mathrm{m}$ long pores were etched in almost 2.5 hours. This results in a mean etching rate of about $0.98 \mu \mathrm{m} \mathrm{min} \mathrm{m}^{-1}$ Next, the samples were thermally oxidized in dry oxygen at $1100{ }^{\circ} \mathrm{C}$ for $190 \mathrm{~min}$. The oxide layer on the wafer backside was patterned by a standard lithography and buffered hydrofluoric acid (BHF) etching. The patterning was required to open the windows where silicon should be removed. After that, the samples were immersed in $25 \mathrm{wt} \%$ TMAH solution at $85^{\circ} \mathrm{C}$ to etch off the backside silicon. Finally, the samples were dipped in $5 \mathrm{wt} \% \mathrm{HF}$ solution to remove the oxide and to create membranes of through-wafer pores in silicon. 


\section{B.2.2 Fabrication of colloidal wires}

Latex (polystyrene - PS) spheres with diameter of $1 \mu \mathrm{m}, 1.2 \mu \mathrm{m}$ and $1.5 \mu \mathrm{m}$ are used to infiltrate the pores. Aqueous dispersions of sulphate stabilized latex spheres were washed with deionized water and ethanol through several cycles of sonication/centrifugation. The surface charge of the employed particles was ca. $0.98 \mathrm{mC} \mathrm{m}^{-2}$. The PS spheres suspended in ethanol were then infiltrated into the silicon membrane by means of vacuum (180 mbar) on a specially constructed setup depicted in figure B.4.

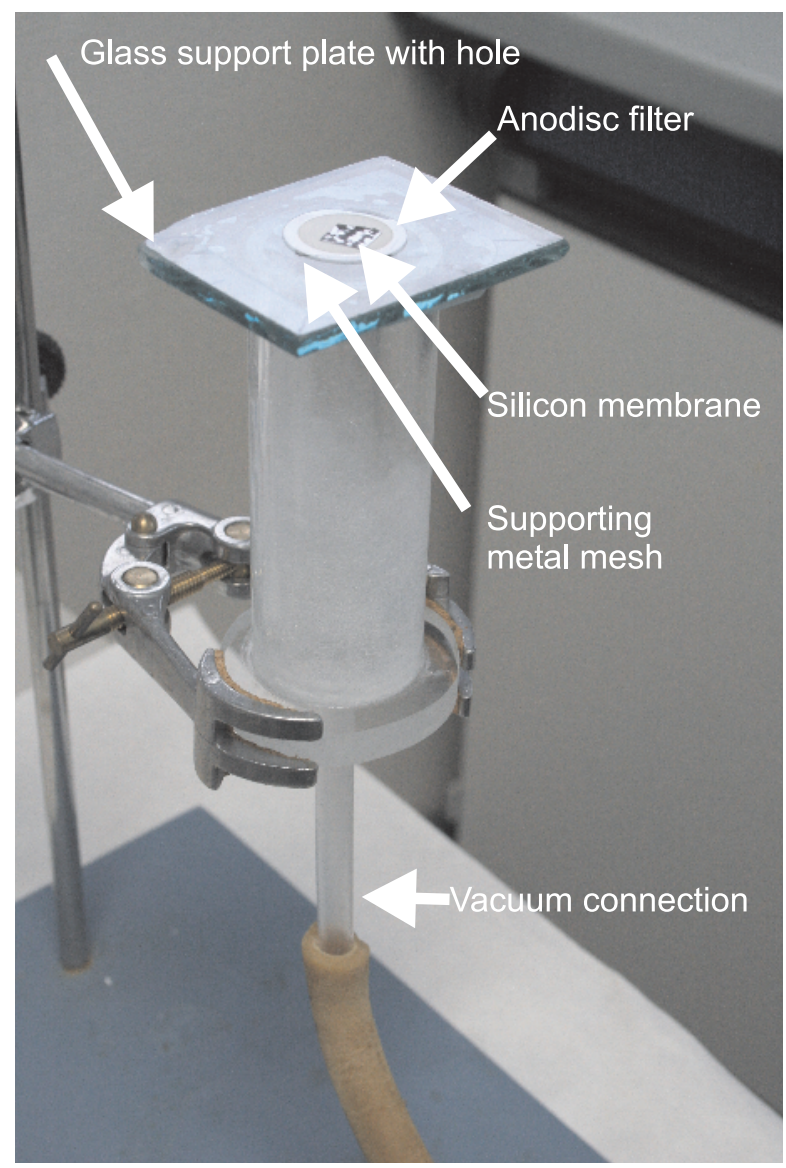

Figure B.4: Setup for infiltrating macroporus silicon membrane with latex spheres. The silicon membrane is placed an Anodics filter which is placed on top on a metal mesh. As a flat support for the membrane and the metal mesh a glass plate with a hole is used. The glass plate is put on a PMMA tube which is connected to vacuum by smaller tubes. 
The setup for infiltrating the macropurous silicon membranes consists of a PMMA tube on which a glass plate with a hole can be placed and its connected to vacuum by a smaller tubes. The sealing between the PMMA tube and the glass plate is provided by a in lab made PDMS film. The glass plate serve as a flat support where the metal mesh and alumina filter can be placed. For infiltrating the silicon membrane was placed on an alumina filter (Anodisc, alumina filter $20 \mathrm{~nm}$ pores) to prevent the spheres to flow out from the pores. A metal mesh (InterNet Inc, microetched Stainless Steel, thickness $0.13 \mathrm{~mm}$, hole size $0.15 \mathrm{~mm}$ ) was used as supporting plate for the Anodisc filter. The infiltration with the PS spheres was repeated several times under vacuum in order to ensure proper infiltration of each pore. Residual ethanol was removed by leaving the infiltrated membrane in an oven $\left(70{ }^{\circ} \mathrm{C}\right)$ for 1 hour. Afterwards, polydimethylsiloxane (PDMS) diluted with n-pentane $(1: 3 \mathrm{v} / \mathrm{v})$ was infiltrated with the same setup mentioned earlier. Then, in order to cure the PDMS, the membrane was place in an oven $\left(70{ }^{\circ} \mathrm{C} / 24\right.$ hours $)$. The silicon membrane is dipped in $5 \% \mathrm{HF}$ solution in order remove the native silica from silicon membrane. Finally the template was removed by etching in Tetramethylammonium hydroxide (TMAH). The solution of the TMAH which contains the CCW's was filtered and analyzed by means of scanning electron microscopy (SEM). A simplified overview of the previously described procedure is depicted in figure B.5.

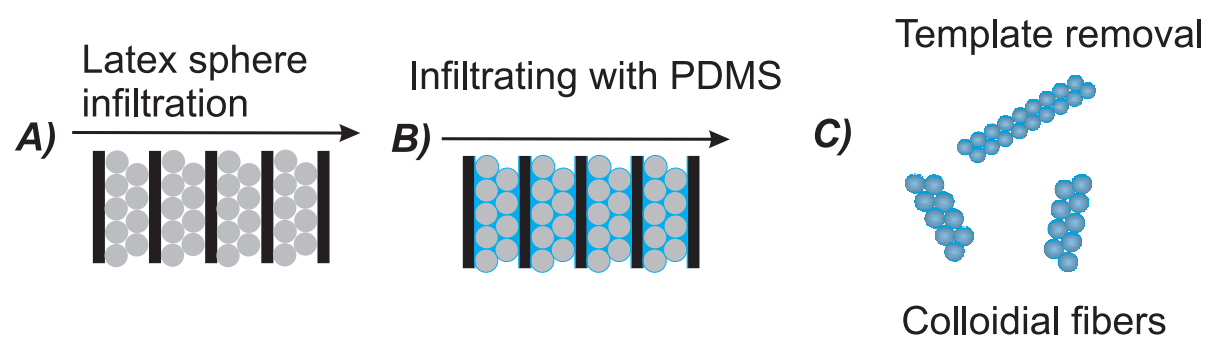

Figure B.5: Scheme of the different steps involved to process CCWs. a) Infiltration of the pores with polystyrene spheres. b) Infiltration of the pores with PDMS. c) The silicon membrane is removed and CCWs are obtained.

Previous procedure took some trial and error to find correct step to obtain CCW with repeatability.

- Finding a dissolvent for PDMS that was light and evaporated quickly. In first trials we used hexane but found it too viscous and evaporated very slowly. Too much PDMS resulted in a fibres with 
PS spheres almost totally embedded in PDMS as showed in figure B.6(a). When we used n-pentane it evaporated quickly and lowered the viscosity of the solution, thus only remaining between the contact point between spheres.

- Before removing the silicon template a dip in $5 \% \mathrm{HF}$ was necessary to remove the native silica. If not done so the latex sphere remained enclosed in a thin native pore silica shell as shown in figure B.6(b).

- Using a fresh batch of PDMS. In some cases when out of date PDMS was used it did not cure properly.

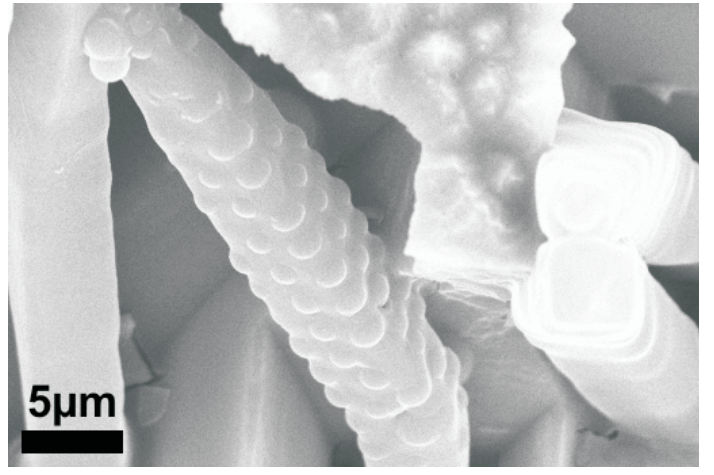

(a)

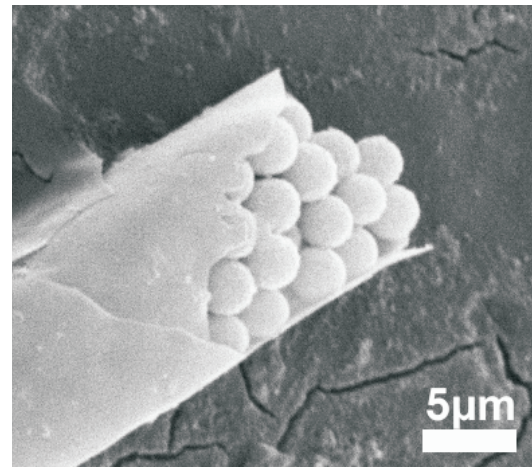

(b)

Figure B.6: Before a procedure for obtaining CCW with good repeatability was known some interesting results from failures where obtained. (a) A CCW fiber infiltrated with PDMS having to large viscosity (b) An example of where $\mathrm{HF}$ acid did not managed to reach and remove the silica layer. 


\section{B.3 Results and discussion}

Figure B.7 shows a SEM image of several pores of the silicon membrane infiltrated with PS particles. As showed in figure B.7 the spheres have a tendency to locate on the pore wall, repelling each other. The repulsion between particles resulting from the negative surface groups $\left(\mathrm{SO}_{4}^{2-}\right)$ of PS spheres (particle charge ca. $0.98 \mathrm{mC} \mathrm{m}^{-2}$ ) should be emphasized. Also, as pores walls are coated with a native thin silica layer, hydrogen-bonding-like interactions between sulphate groups of particles and silanols moieties of the silica walls induce attractive forces. As a matter of fact, it can be observed that PS spheres preferably distribute themselves equidistantly in the equatorial plane.

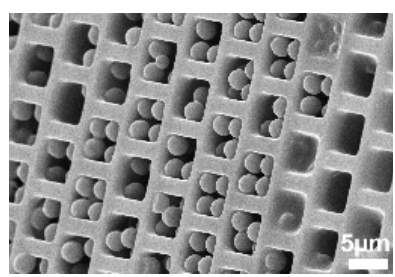

(a)

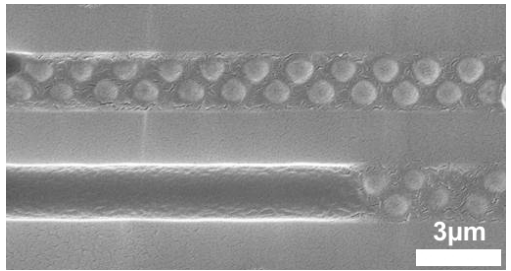

(b)

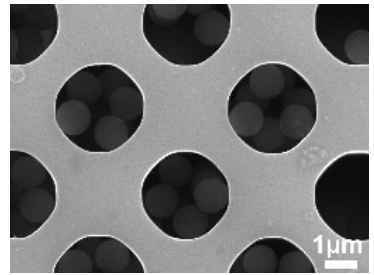

(c)

Figure B.7: (a) Entrance pores of macroporus silicon membrane infiltrated with latex spheres. Not all pores have been properly infiltrated by PS spheres. Spheres show a tendency to adhere to the walls of the pore. (b) View of cut through macroporus silicon membrane infiltrated with latex spheres. Again one of the pores is not completely filled with PS spheres (c) Exit pores of a macroporus silicon membrane infiltrated with PS spheres. The ordering and adherence to the pore wall is clearly visible.

Finally, once the silicon template was removed, fibers made of PS spheres embedded in PDMS were obtained, as it shown in figure B.8. Fibers are found with different particle ordering, length and fiber thickness.

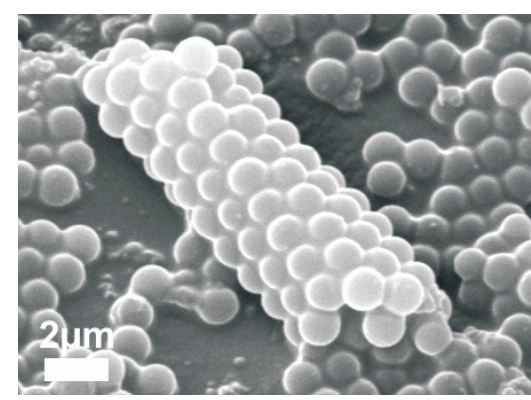

(a)

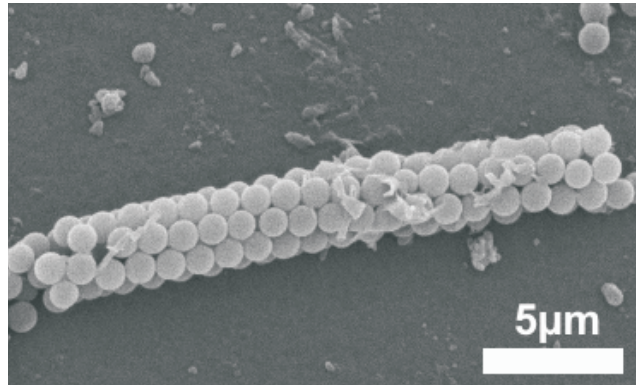

(b)

Figure B.8: Figure continue on the next page... 


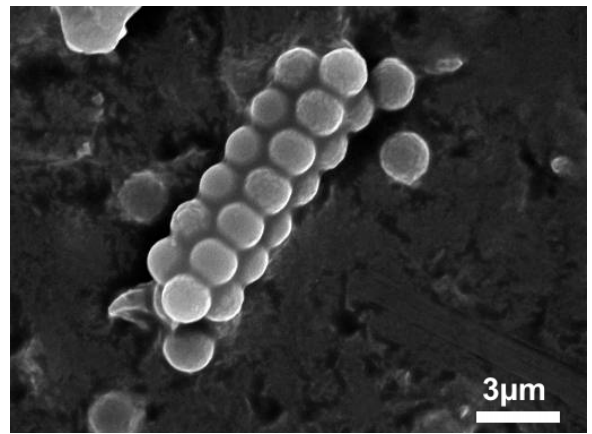

(c)

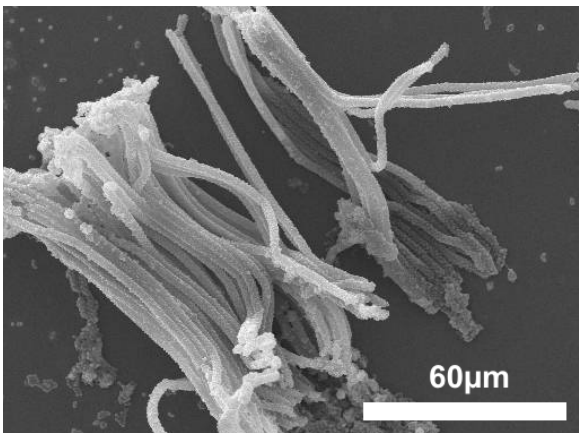

(d)

Continued Figure B.8: Different CCW fibers obtained after removing the silicon template. The length of the CCW fibers ranges from few microns as in image a), b) and c) up to tens of microns like image d).

We have studied the different particle arrangements as the $D$ factor changes gradually. Presenting a realistic packing model that takes into account the particle repulsion and the interaction with the pore wall is a formidable task.

We will use a very naïve simple hard sphere model [27], as a guide, to understand both particle ordering as well as the importance of the charge repulsion. To emulate experimental results, spheres in the model have the tendency to adhere to the surface of the pore. For $D<2$ our model gives results identical to those reported by Pickett et al. [27]. However for $D>2$, sphere affinity for the silica wall surface of the pore induces new particle arrangements not predicted before. In what follows we will report experimental results for different $D$ values in the region $1<D<3$ and discuss them in light of our packing model. Figure B.9 shows both SEM images and the corresponding models of colloidal fibers for different values of the $D$ parameter. For small $D$ values the system orders in single and double strings of spheres, that are not mechanically stable. Indeed, a zigzag type structure (figure B.9(a)) of $1.5 \mu \mathrm{m}$ diameter latex spheres appears for $D=1.4$. The obtained results show similarities to those obtained by the so-called phase I shown in the hard-spheres model by Pickett et al. [27], For a value $D=1.9$ our model predicts a chiral structure. It consists of two strings of spheres with helixlike shape winding around each other. This structure is very difficult to observe experimentally. The closest example of this string ordering can be seen in figure B.9(b), where a helix turn in the CCW can be guessed. However, 
we can not provide clear examples of chiral order. As we have seen in SEM images of figure B.7, Coulomb interaction between spheres can play an important role. If particle repulsion dominates particles would tend to repel each other, and probably chiral order would not be favored. It can be understood by the following naïve description. The hard-sphere model shows a double-sphere string winding each other. Neighboring particles in each string are in contact. Also, neighboring spheres from different strings are not located in the equatorial plane of the pore. If particle repulsion dominates, spheres would not adopt such a close-packed order in each string and chirality could not be induced. More experiments with strict control of the particle charge would be necessary to understand the influence of Coulomb interaction on the particle ordering of CCWs. Figure B.9(c) shows both an SEM image and a particle arrangement model for $D=2$. A string configuration appears that consists of achiral doublets composed by sphere dimmers that fit along the diameter of the channel. Spheres order with respect to each other, with an angle of $\pi / 2$ between neighbor dimmers axis. For $D$ values above 2, the sphere tendency to attach to the pore surface and the interparticle repulsive forces can induce the appearance of a coaxial cylindrical hole in the center of the structure, For instance, when $D=2.2$, spheres pack in a squarelike prism with four spheres in the base (figure B.9(d)), and one can guess a small hole in the center of the fiber. The hollow core in the fibers is a consequence of the preference of colloidal particles to attach to the membrane wall. This fact can be more clearly seen in fibers with larger $D$ values. For example, in a sample with $D=2.7$ (figure B.9(e)) a central axial hole can be seen if the subsequent polymer infiltration is not complete. When a full polymer infiltration is achieved, a central polymer fiber surrounded by ordered colloidal particles is obtained. To our knowledge, such a type of hollow colloidal fiber has not previously been predicted by theoretical models. However, Li et al. [35, 36] have shown similar fiberlike structures for silica spheres infiltrated into silicon membranes. Therefore particle repulsion plays a very important role and it is more visible for large values of the $D$ factor. For example, figure B.9(f) illustrates both an SEM image and model of the particle arrangement for $D=2.9$. The SEM image shows a CCW composed of six helices winding around a central hole where particles arrange in a non-close packed manner. Our model shows that spheres tend to attach themselves to the pore surface, but they do not cover completely the pore surface. Particle repulsion would induce an isotropic coverage of latex 
particles on the pore surface, and therefore spheres will be isotropically distributed in a non-close-packed manner as seen in the SEM image of figure B.9(f).
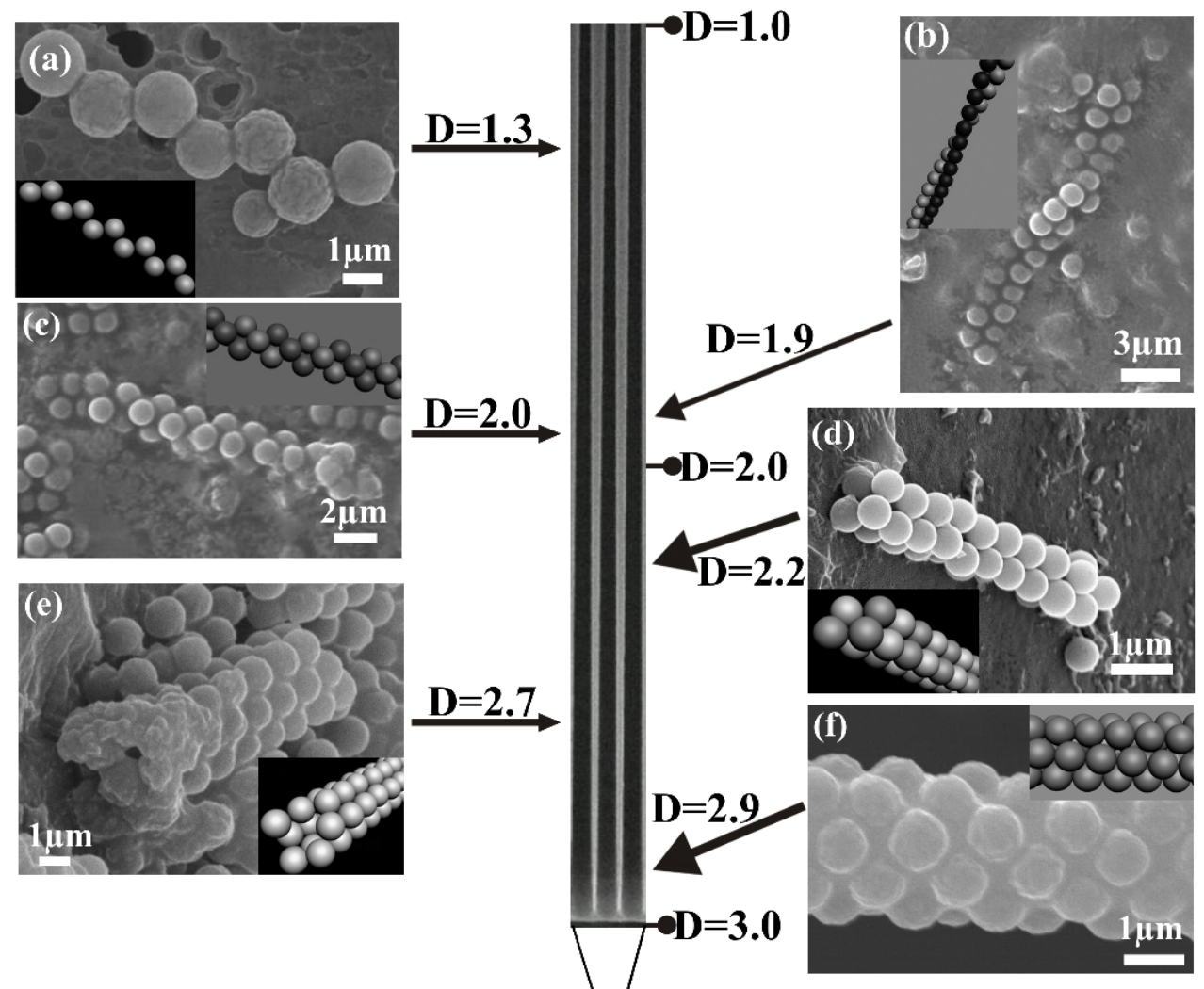

Figure B.9: CCW SEM images for different values of the $D$ parameter. The central image (not to scale and only for orientation purposes) visualizes exaggeratedly the change of the pore diameter along the vertical direction. The corresponding $\mathrm{D}$ parameter and the sphere size $(\phi)$ of the different images are the following: (a) $D=1.4 ; \phi=1.5 \mu \mathrm{m}$. (b) $D=1.9 ; \phi=1.2 \mu \mathrm{m}$. (c) $D=2.1 ; \phi=1.2 \mu \mathrm{m}$. (d) $D=2.3 ; \phi=1.2 \mu \mathrm{m}$. (e) $D=2.7 ; \phi=1.0 \mu \mathrm{m}$. (f) $D=2.9 ; \phi=1.0 \mu \mathrm{m}$. The insets of the SEM pictures show the results of the model. 


\section{B.4 Conclusions}

We have shown a method to produce CCWs with a strict control of the $D$ parameter. We show a manifold variety of particle arrangements in a wire like manner for different values of the $D$ factor ranging between 1 and 3. Coulomb repulsion between particles play very important role in the particle ordering. More experiments with a strict control of the particle charge and a model including Coulomb repulsion between particles will help to understand particle ordering and, more important, the appearance of chiral order in CCWs. These types of conformations could found interesting applications in the design of new capillary columns for chromatographic applications. Finally, colloids can again be used as a model, at the mesoscale range, of particle ordering in the nanometer regime [32]. 


\section{Bibliography}

[1] A. Yethiraj and A. van Blaaderen. "A colloidal model system with an interaction tunable from hard sphere to soft and dipolar". Nature, 421(6922):513-517, 2003.

[2] K. N. Pham, A. M. Puertas, J. Bergenholtz, S. U. Egelhaaf, A. Moussaid, P. N. Pusey, A. B. Schofield, M. E. Cates, M. Fuchs, and W. C. K. Poon. "Multiple glassy states in a simple model system". Science, 296(5565):104-106, 2002.

[3] P. N. Pusey and W. van Megen. "Phase behaviour of concentrated suspensions of nearly hard colloidal spheres". Nature, 320(6060):340-342, 1986.

[4] C. López. "Materials aspects of photonic crystals". Advanced Materials, 15(20):1679-1704, 2003.

[5] O. D. Velev and A. M. Lenhoff. "Colloidal crystals as templates for porous materials". Current Opinion in Colloid $\&$ Interface Science, 5(1-2):56-63, 2000.

[6] J. H. Holtz and S. A. Asher. "Polymerized colloidal crystal hydrogel films as intelligent chemical sensing materials". Nature, 389(6653):829-832, 1997.

[7] L. V. Woodcock. "Entropy difference between the face-centred cubic and hexagonal close-packed crystal structures". Nature, 385(6612):141-143, 1997.

[8] H. Míguez, F. Meseguer, C. López, A. Mifsud, J. S. Moya, and L. Vazquez. "Evidence of FCC crystallization of $\mathrm{SiO}_{2}$ nanospheres". Langmuir, 13(23):6009-6011, 1997.

[9] Edited By R K Chang and A J Campillo. Optical Processes In Microcavities. World Scientific, Singapore, 1996.

[10] Pawel Pieranski, L. Strzelecki, and B. Pansu. "Thin Colloidal Crystals". Physical Review Letters, 50(12):900, 1983.

[11] S. M. Ilett, A. Orrock, W. C. K. Poon, and P. N. Pusey. "Phase behavior of a model colloid-polymer mixture". Physical Review E, 51(2):1344, 1995. 
[12] Matthias Schmidt and Hartmut Löwen. "Freezing between Two and Three Dimensions". Physical Review Letters, 76(24):4552, 1996.

[13] S. Neser, C. Bechinger, P. Leiderer, and T. Palberg. "Finite-Size Effects on the Closest Packing of Hard Spheres". Physical Review Letters, 79(12):2348, 1997.

[14] I. Cohen, T. G. Mason, and D. A. Weitz. "Shear-induced configurations of confined colloidal suspensions". Physical Review Letters, 93(4):4, 2004.

[15] Eugenia Kumacheva, Piotr Garstecki, Hongkai Wu, and George M. Whitesides. "Two-Dimensional Colloid Crystals Obtained by Coupling of Flow and Confinement". Physical Review Letters, 91(12):128301, 2003.

[16] E. Kumacheva, R. K. Golding, M. Allard, and E. H. Sargent. "Colloid crystal growth on mesoscopically patterned surfaces: Effect of confinement". Advanced Materials, 14(3):221, 2002.

[17] M. Allard, E. H. Sargent, P. C. Lewis, and E. Kumacheva. "Colloidal crystals grown on patterned surfaces". Advanced Materials, 16(15):1360, 2004.

[18] J. P. Hoogenboom, C. Retif, E. de Bres, M. V. de Boer, A. K. van Langen-Suurling, J. Romijn, and A. van Blaaderen. "Templateinduced growth of close-packed and non-close-packed colloidal crystals during solvent evaporation". Nano Letters, 4(2):205-208, 2004.

[19] E. Kim, Y. N. Xia, and G. M. Whitesides. "Micromolding in capillaries: Applications in materials science". Journal of the American Chemical Society, 118(24):5722-5731, 1996.

[20] H. Yang, P. Deschatelets, S. T. Brittain, and G. M. Whitesides. "Fabrication of High Performance Ceramic Microstructures from a Polymeric Precursor Using Soft Lithography". Advanced Materials, 13(1):54-58, 2001.

[21] H. Miguez, S. M. Yang, N. Tetreault, and G. A. Ozin. "Oriented free-standing three-dimensional silicon inverted colloidal photonic crystal microribers". Advanced Materials, 14(24):1805-1808, 2002. 
[22] Y. Lin, P. R. Herman, C. E. Valdivia, J. Li, V. Kitaev, and G. A. Ozin. "Photonic band structure of colloidal crystal self-assembled in hollow core optical fiber". Applied Physics Letters, 86(12):3, 2005.

[23] U. Kamp, V. Kitaev, G. von Freymann, G. A. Ozin, and S. A. Mabury. "Colloidal crystal capillary columns - Towards optical chromatography". Advanced Materials, 17(4):438, 2005.

[24] Y. D. Yin and Y. N. Xia. "Self-assembly of spherical colloids into helical chains with well-controlled handedness". Journal of the American Chemical Society, 125(8):2048-2049, 2003.

[25] Y. D. Yin, Y. Lu, B. Gates, and Y. N. Xia. "Template-assisted selfassembly: A practical route to complex aggregates of monodispersed colloids with well-defined sizes, shapes, and structures". Journal of the American Chemical Society, 123(36):8718-8729, 2001.

[26] R. O. Erickson. "Tubular packing of spheres in biological finestructure". Science, 181(4101):705-716, 1973.

[27] G. T. Pickett, M. Gross, and H. Okuyama. "Spontaneous chirality in simple systems". Physical Review Letters, 85(17):3652-3655, 2000.

[28] H. K. Wu, V. R. Thalladi, S. Whitesides, and G. M. Whitesides. "Using hierarchical self-assembly to form three-dimensional lattices of spheres". Journal of the American Chemical Society, 124(48):1449514502, 2002.

[29] Fraser Gray and Christopher Viney. "Dense packing of spheres around rods in supramolecular aggregates". European Journal of Physics, 21:6099, 2000.

[30] Lubert Stryer. Biochemistry. W. H. Freeman and Company, New York, 1998.

[31] J. H. Moon, S. Kim, G. R. Yi, Y. H. Lee, and S. M. Yang. "Fabrication of ordered macroporous cylinders by colloidal templating in microcapillaries". Langmuir, 20(5):2033-2035, 2004.

[32] A. N. Khlobystov, D. A. Britz, A. Ardavan, and G. A. D. Briggs. "Observation of ordered phases of fullerenes in carbon nanotubes". Physical Review Letters, 92(24):245507, 2004. 
[33] J. G. Huang and T. Kunitake. "Latex particle-encapsulated titania/polymer composite nanotubings: free-standing, one-dimensional package of colloidal particles". Chemical Communications, (21):2680-2682, 2005.

[34] J. Huang and T. Kunitake. "Nanotubings of titania/polymer composite: template synthesis and nanoparticle inclusion". Journal of Materials Chemistry, 16(43):4257-4264, 2006.

[35] F. Li, J. B. He, W. L. L. Zhou, and J. B. Wiley. "Synthesis of porous wires from directed assemblies of nanospheres". Journal of the American Chemical Society, 125(52):16166-16167, 2003.

[36] F. Li, X. Badel, J. Linnros, and J. B. Wiley. "Fabrication of colloidal crystals with tubular-like packings". Journal of the American Chemical Society, 127(10):3268-3269, 2005.

[37] T. Trifonov, L. F. Marsal, A. Rodriguez, J. Pallares, and R. Alcubilla. "Fabrication of two- and three-dimensional photonic crystals by electrochemical etching of silicons". Physica Status Solidi C, 2(8):3104-3107, 2005.

[38] T. Trifonov, A. Rodriguez, F. Servera, L. F. Marsal, J. Pallares, and R. Alcubilla. "High-aspect-ratio silicon dioxide pillars". Physica Status Solidi A, 202(8):1634-1638, 2005.

[39] V. Lehmann. "The Physics of Macropore Formation in Low Doped n-Type Silicon". Journal of The Electrochemical Society, 140(10):2836-2843, 1993. 


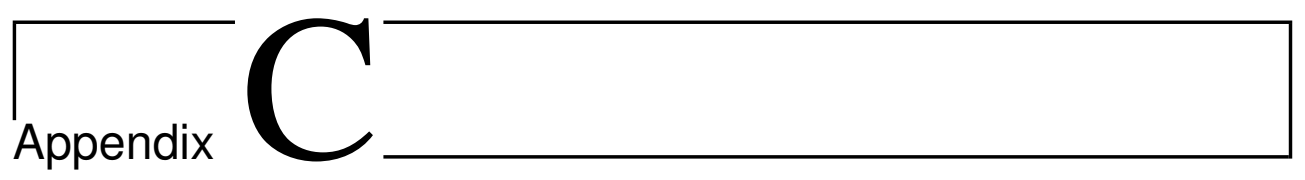

\section{Acronyms}

\begin{tabular}{llc} 
Abbreviation & Description & Page \\
\hline AFM & Atomic Force Microscope & 52 \\
CVD & Chemical Vapor Deposition & 25 \\
FF & Filling Fraction & 86 \\
FTIR & Fourier Transform InfraRed spectroscopy & 47 \\
FWHM & Full Width Half Maximum & 60 \\
HRTEM & High Resolution Transmission Electron Microscope & 46 \\
IR & Infra Red (radiation around 1 $\mu m$ and beyond) & 82 \\
KBr & Potassium Bromide & 87 \\
RF & Radio Frequency & 25 \\
SEM & Scanning Electron Microscope & 52 \\
Si & Silicon & 6 \\
XRD & X-Ray powder Diffraction & 41 \\
VCSEL & Vertical Cavity Surface Emitting Laser & 16
\end{tabular}

